\title{
Why are patients dissatisfied following a total knee replacement? A systematic review
}

\author{
Naoki Nakano ${ }^{1,2} \cdot$ Haitham Shoman ${ }^{1} \cdot$ Fernando Olavarria $^{1} \cdot$ Tomoyuki Matsumoto $^{2} \cdot$ Ryosuke Kuroda $^{2} \cdot$ \\ Vikas Khanduja ${ }^{1}$ (D)
}

Received: 1 October 2019 / Accepted: 6 May 2020 / Published online: 8 July 2020

(C) The Author(s) 2020

\begin{abstract}
Background Although total knee replacement (TKR) is an effective intervention for end-stage arthritis of the knee, a significant number of patients remain dissatisfied following this procedure. Our aim was to identify and assess the factors affecting patient satisfaction following a TKR.

Materials and methods In accordance with the PRISMA guidelines, two reviewers searched the online databases for literature describing factors affecting patient satisfaction following a TKR. The research question and eligibility criteria were established a priori. Any clinical outcome study that described factors relating to overall satisfaction after primary TKR was included. Quality assessment for the included studies was performed by two accredited orthopaedic surgeons experienced in clinical research.

Results The systematic review identified 181 relevant articles in total. A history of mental health problems was the most frequently reported factor affecting patient satisfaction (13 reportings). When the results of the quality assessment were taken into consideration, a negative history of mental health problems, use of a mobile-bearing insert, patellar resurfacing, severe pre-operative radiological degenerative change, negative history of low back pain, no/less post-operative pain, good post-operative physical function and preoperative expectations being met were considered to be important factors leading to better patient satisfaction following a TKR. Conclusion Surgeons performing a TKR should take these factors into consideration prior to deciding whether a patient is suitable for a TKR. Secondarily, a detailed explanation of these factors should form part of the process of informed consent to achieve better patient satisfaction following TKR. There is a great need for a unified approach to assessing satisfaction following a TKR and also the time at which satisfaction is assessed.
\end{abstract}

Keywords Total knee replacement · Total knee arthroplasty · Satisfaction · Dissatisfaction · Systematic review

\section{Introduction}

Total knee replacement (TKR) is one of the most effective surgical interventions for relief of pain and functional

Electronic supplementary material The online version of this article (https://doi.org/10.1007/s00264-020-04607-9) contains supplementary material, which is available to authorized users.

Vikas Khanduja

vk279@cam.ac.uk

Naoki Nakano

gnaokix1981@gmail.com

Haitham Shoman

haitham.sh89@gmail.com

Fernando Olavarria

olavarria.f@gmail.com

Tomoyuki Matsumoto

matsun@m4.dion.ne.jp recovery in patients with advanced osteoarthritis $(\mathrm{OA})$ of the knee. Management of OA costs the UK economy equivalent to $1 \%$ of its gross national product per year [1]. In the USA, the annual number of TKRs has been projected to rise by over
Ryosuke Kuroda

kurodar@med.kobe-u.ac.jp

1 Department of Trauma and Orthopaedics,

Addenbrooke's - Cambridge University Hospitals NHS Foundation Trust, Box 37, Hills Road, Cambridge CB2 0QQ, UK

2 Department of Orthopaedic Surgery, Kobe University Graduate School of Medicine, 7-5-1 Kusunoki-cho, Chuo-ku, Kobe 650-0017, Japan 
Table 1 Inclusion and exclusion criteria applied to articles identified in the literature

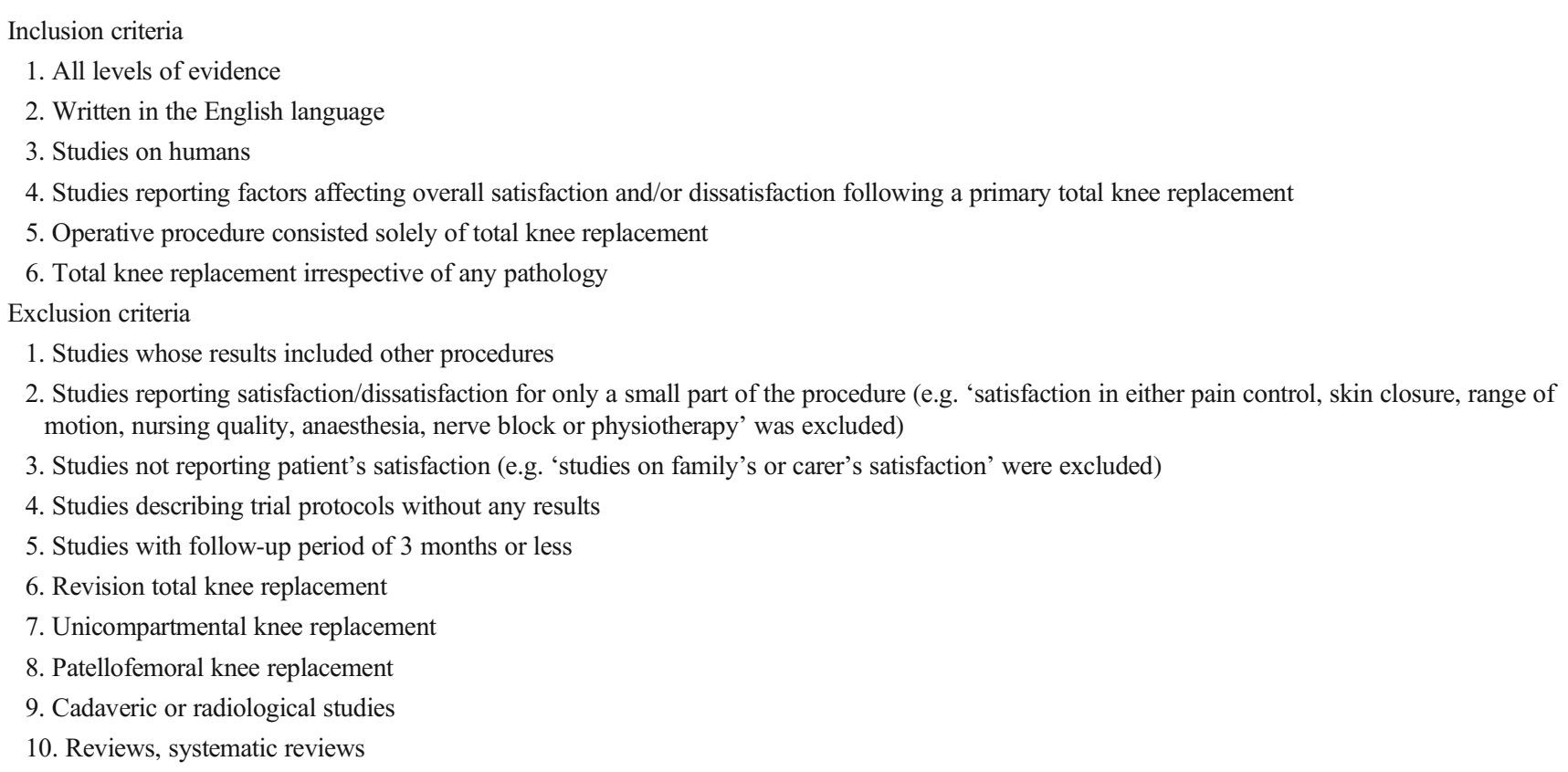

$670 \%$ to 3.48 million cases by 2030 [2]. Outcomes of TKR are traditionally assessed by survival analysis with revision as the end point, and technical outcomes of this intervention are excellent. According to the UK National Joint Registry (NJR) annual report, the survival rate has been reported to be over $99.5 \%$ after one year and $95.6 \%$ at ten years [3].

A revision TKR is most commonly performed for loosening, fracture or infection. However, survival analysis tends to underestimate poor function, pain or dissatisfaction because these problems do not necessarily lead to a revision and are not recorded in the registry. Another issue is that reporting of the outcome of a TKR has predominantly been based on surgeon-derived outcome measures, which include range of movement (ROM), joint stability and post-operative alignment [4-6]. However, a report identified a poor correlation between surgeon-derived and patient-reported outcomes, with surgeons overestimating outcomes in comparison with the patients' [7]. This correlates well with the fact that a significant number of patients experience continual pain and functional disability and therefore remain dissatisfied following the procedure [8-10].

In the largest ever reported series on satisfaction following a TKR, which included a survey of 27,372 patients, $17 \%$ of the unrevised patients were either dissatisfied or uncertain regarding their outcome [11]. Baker et al. [12] also reviewed the data from

Table 2 Search strategy for Medline

\begin{tabular}{|c|c|c|}
\hline No. & Searches & $\begin{array}{l}\text { Medline } \\
\text { results }\end{array}$ \\
\hline 1 & $\begin{array}{l}\text { satis } \$ \text {.mp. [mp=title, abstract, original title, name of substance word, subject heading word, keyword heading word, protocol } \\
\text { supplementary concept word, rare disease supplementary concept word, unique identifier, synonyms] }\end{array}$ & 366,508 \\
\hline 2 & $\begin{array}{l}\text { tkr.mp. [mp=title, abstract, original title, name of substance word, subject heading word, keyword heading word, protocol } \\
\text { supplementary concept word, rare disease supplementary concept word, unique identifier, synonyms] }\end{array}$ & 1908 \\
\hline 3 & $\begin{array}{l}\text { tka.mp. [mp=title, abstract, original title, name of substance word, subject heading word, keyword heading word, protocol } \\
\text { supplementary concept word, rare disease supplementary concept word, unique identifier, synonyms] }\end{array}$ & 8888 \\
\hline 4 & $\begin{array}{l}\text { "total knee arthroplasty".mp. [mp=title, abstract, original title, name of substance word, subject heading word, keyword heading } \\
\text { word, protocol supplementary concept word, rare disease supplementary concept word, unique identifier, synonyms] }\end{array}$ & 15,890 \\
\hline 5 & $\begin{array}{l}\text { "total knee replacement".mp. [mp=title, abstract, original title, name of substance word, subject heading word, keyword heading } \\
\text { word, protocol supplementary concept word, rare disease supplementary concept word, unique identifier, synonyms] }\end{array}$ & 5129 \\
\hline 6 & 2 or 3 or 4 or 5 & 21,446 \\
\hline 7 & $\begin{array}{l}\text { dissatisf } \$ . m p \text {. [mp=title, abstract, original title, name of substance word, subject heading word, keyword heading word, protocol } \\
\text { supplementary concept word, rare disease supplementary concept word, unique identifier, synonyms] }\end{array}$ & 17,906 \\
\hline 8 & 1 or 7 & 374,612 \\
\hline 9 & 6 and 8 & 2187 \\
\hline
\end{tabular}




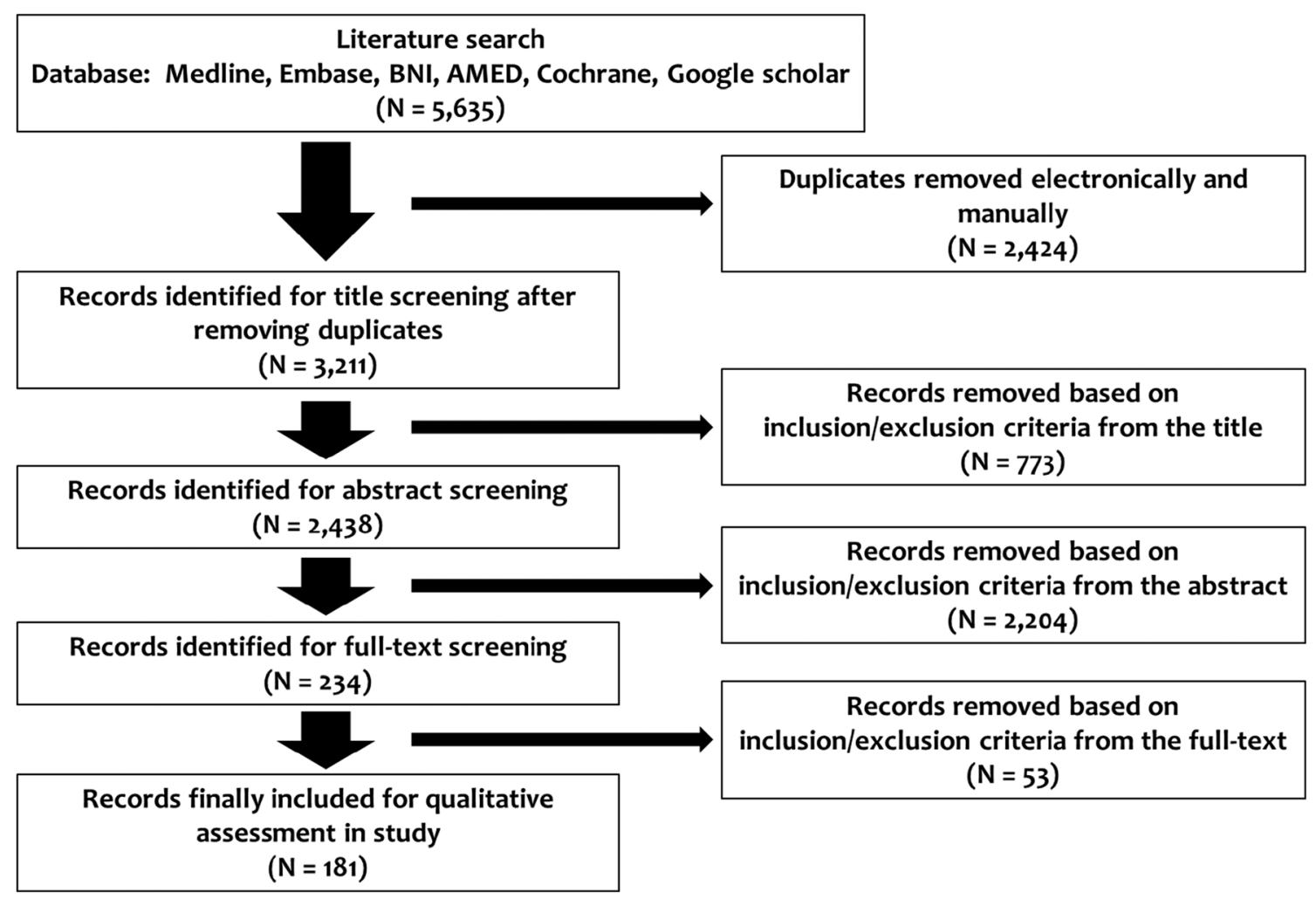

Fig. 1 PRISMA flowchart for results of the literature database search

the NJR in the UK and reported that $71 \%$ of the patients experienced improvement of knee symptoms, but only $22 \%$ of them rated the results as excellent. Therefore, although the surgeonreported outcomes may be good and the patient has no indication for a revision, they may still be dissatisfied following their index TKR. This may be due to a multitude of reasons, but to the best of our knowledge, there has been no systematic review which has specifically focused on the factors that affect patient satisfaction following a TKR. The aim of this systematic review, therefore, was to identify and assess the factors affecting patient satisfaction following a TKR.

\section{Methods}

The protocol of this systematic review was developed and has been registered in the International Prospective Register of Systematic Reviews (PROSPERO 2017 CRD42017084659). The Preferred Reporting Items for Systematic Reviews and Meta-Analyses (PRISMA) guidelines were used for designing this study [13].

\section{Search strategy}

Two accredited orthopaedic surgeons experienced in clinical research searched the online database Medline, Embase, BNI, AMED, Cochrane and Google Scholar for literature relating to satisfaction following a TKR. The PICO (participants, interventions, comparators, outcomes) tool was adopted and modified to formulate the research question and establish the inclusion and exclusion criteria. Selected articles were then exported to Mendeley reference manager software to organise screen and select articles.

\section{Study screening and selection}

Clinical outcome studies that described the factors relating to the overall or general satisfaction/dissatisfaction following a primary TKR irrespective of any pathology were included. The inclusion and exclusion criteria are described in Table 1. Any discrepancies at the title and abstract revision stage were resolved by automatic inclusion to ensure thoroughness. Any discrepancies at the full-text stage were resolved by consensus between the two reviewers. If a consensus could not be reached, a third, more senior reviewer was consulted to resolve the discrepancy.

\section{Data extraction and analysis}

The two reviewers independently extracted relevant study data from the final pool of included articles and recorded this data on a spreadsheet designed a priori in Microsoft Excel 2013 (Microsoft Corporation, Redmond, WA, USA). The quality of studies including bias was then analysed and 


\begin{tabular}{|c|c|c|c|c|c|c|c|}
\hline $3^{\circ}$ & ¿ $\mathbb{z}$ & $\tilde{\infty}$ & if & $\stackrel{\infty}{n}$ & $\infty$ & $5 \overline{5}$ & $\stackrel{\infty}{\curvearrowright}$ \\
\hline$\sum^{\overline{0}}$ & 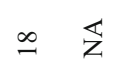 & m & ते : & $\Xi$ & $m$ & $\stackrel{n}{n}$ & $\stackrel{n}{\infty}$ \\
\hline 言 & $\cong \stackrel{ \pm}{\stackrel{\sim}{\sim}}$ & $\stackrel{\infty}{=}$ & $\pm \stackrel{\infty}{\triangle}$ & $\stackrel{ \pm}{ }$ & 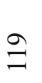 & 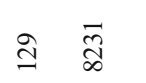 & 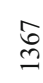 \\
\hline 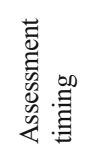 & 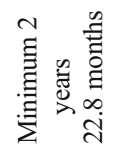 & 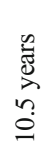 & 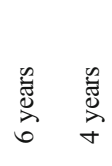 & 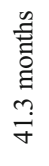 & 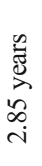 & 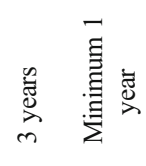 & 㺃 \\
\hline 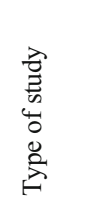 & 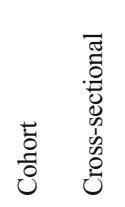 & 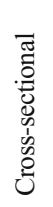 & 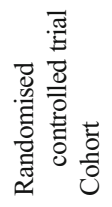 & $\begin{array}{l}\frac{t}{0} \\
\frac{0}{0}\end{array}$ & $\begin{array}{l}\frac{t}{0} \\
\text { o }\end{array}$ & 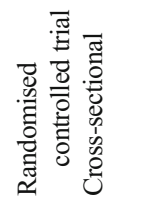 & $\begin{array}{l}5 \\
\text { 융 }\end{array}$ \\
\hline
\end{tabular}

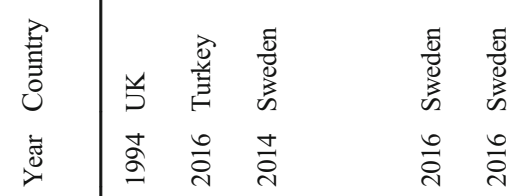

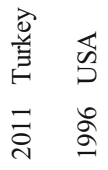

究

光 光

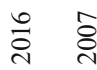

롱

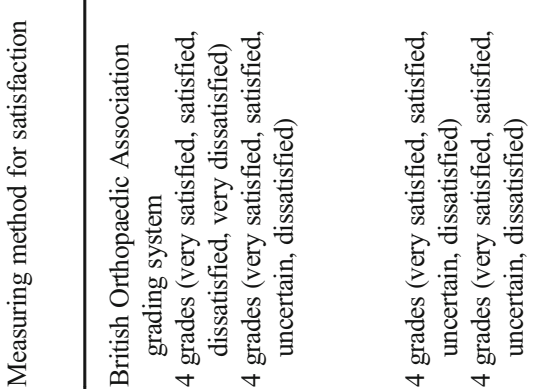

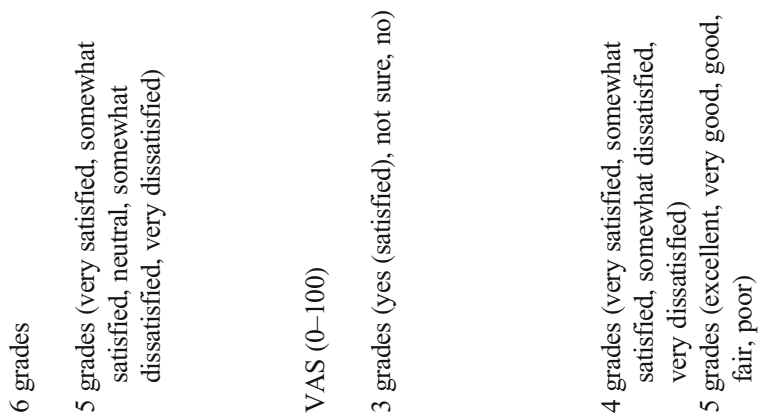

西

逮

子

(1)

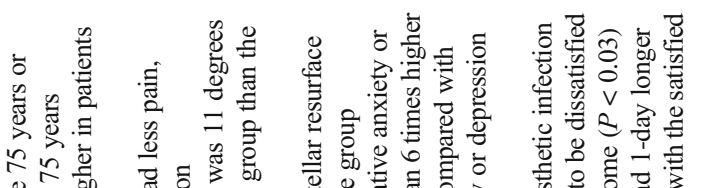

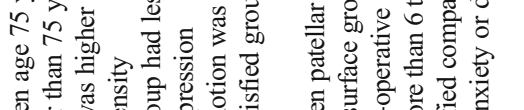

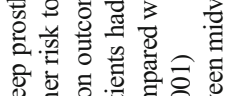

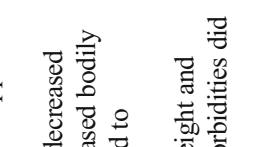

(1)

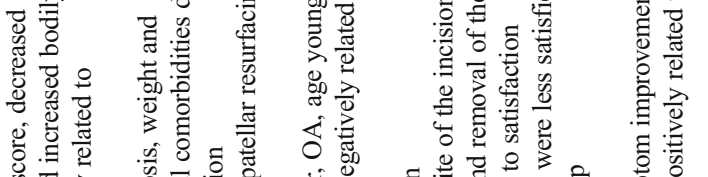

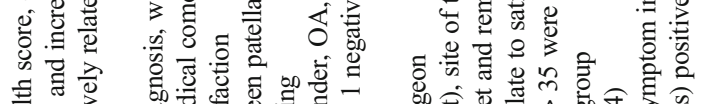

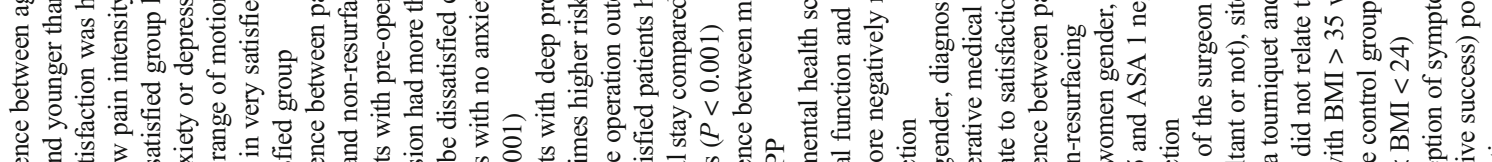

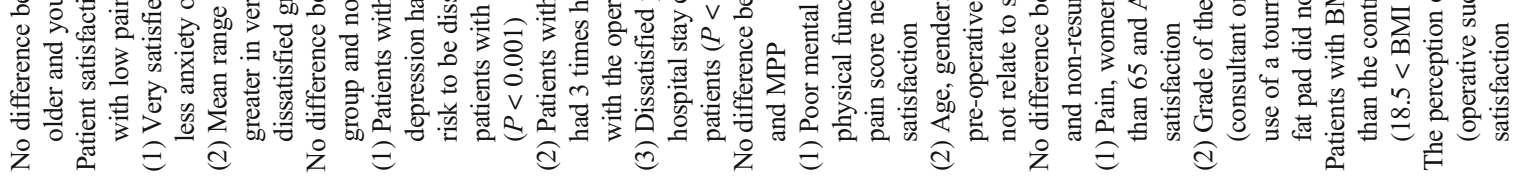

$\stackrel{\square}{.}$

$\bar{\infty}$

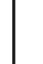

高

离

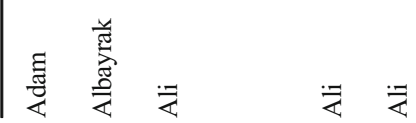

衰变

咅

言章 


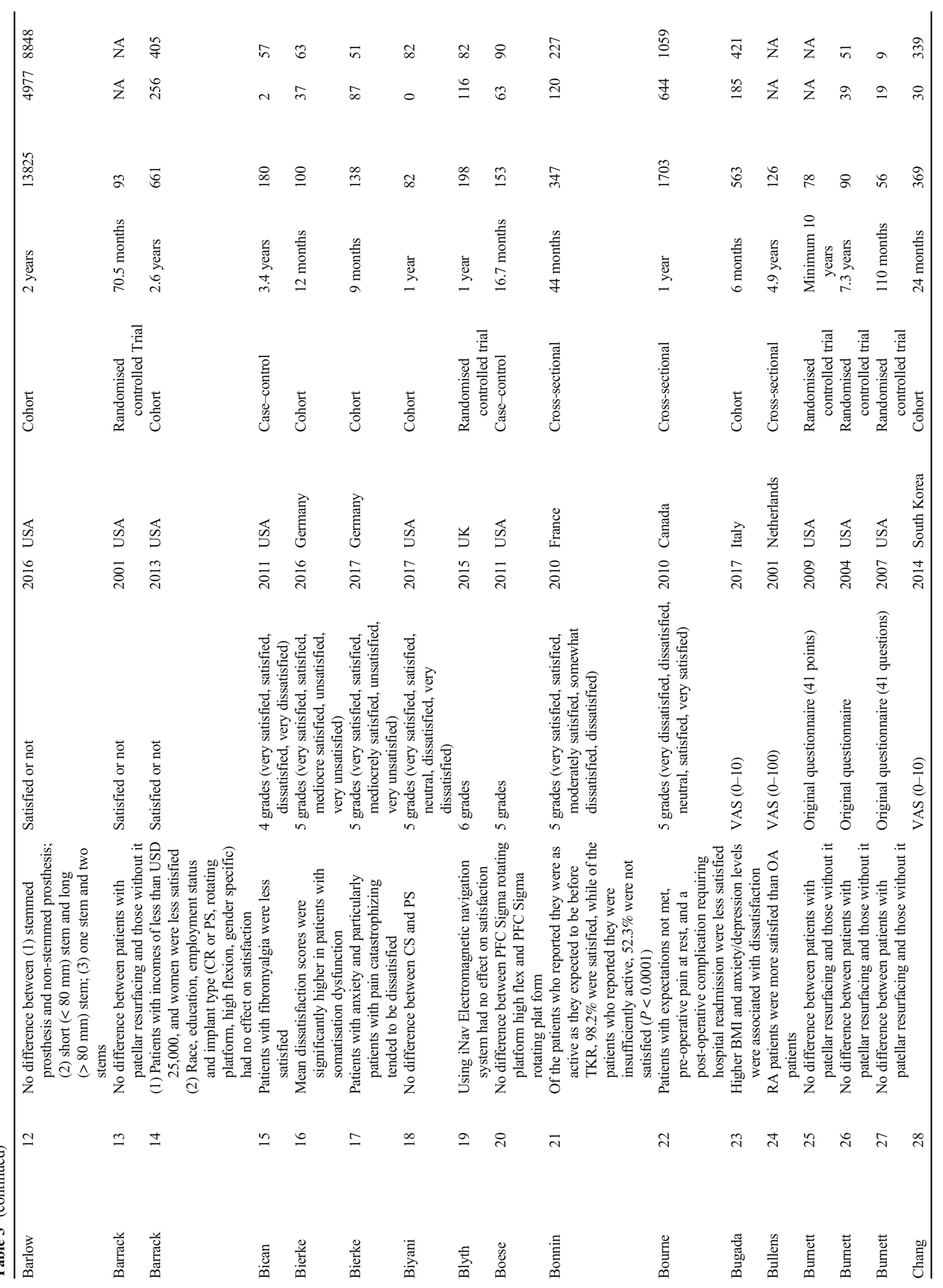




\begin{tabular}{|c|c|c|c|c|c|c|c|c|c|c|c|c|}
\hline in & $\stackrel{9}{\exists}$ & $\infty$ & \begin{tabular}{ll}
\multirow{H}{n}{} & $\stackrel{n}{m}$ \\
$m$
\end{tabular} & $\stackrel{n}{m}$ & 䓌 & $\stackrel{\check{m}}{m}$ & 嵒 & $\Xi$ & $\stackrel{ \pm}{2}$ & i & ते & $\ddot{\theta}$ \\
\hline$\stackrel{m}{m}$ & $a$ & $\simeq$ & $\stackrel{\infty}{\vec{\sigma}} \stackrel{\bar{\Xi}}{\underline{a}}$ & $\stackrel{ \pm}{\stackrel{ \pm}{\Xi}}$ & ฉ̊ & $\underline{\bar{\sigma}}$ & $\vec{q}$ & $\bar{\beth}$ & $\stackrel{\infty}{\simeq}$ & 8 & 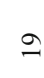 & $\approx$ \\
\hline
\end{tabular}

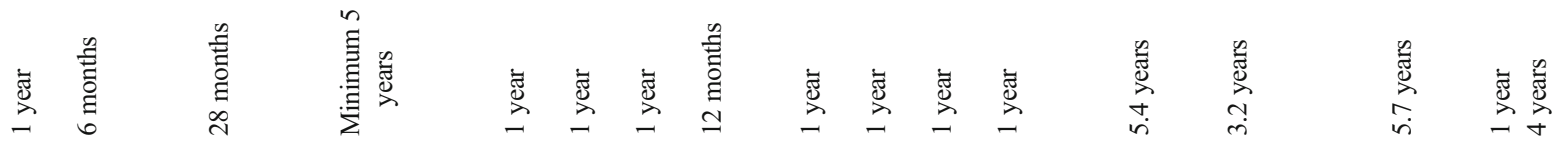

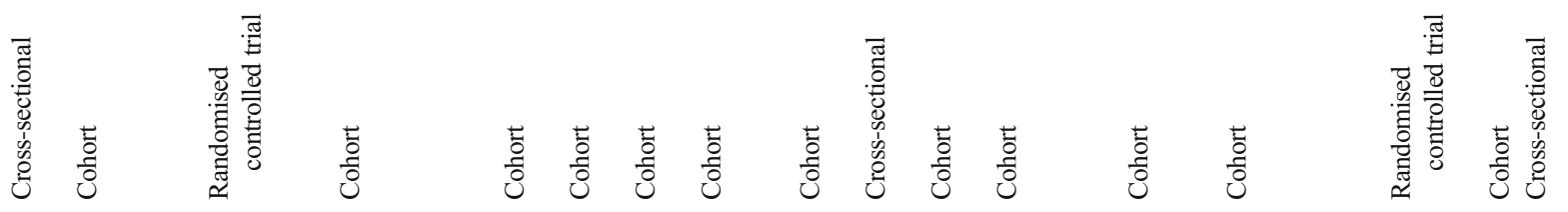

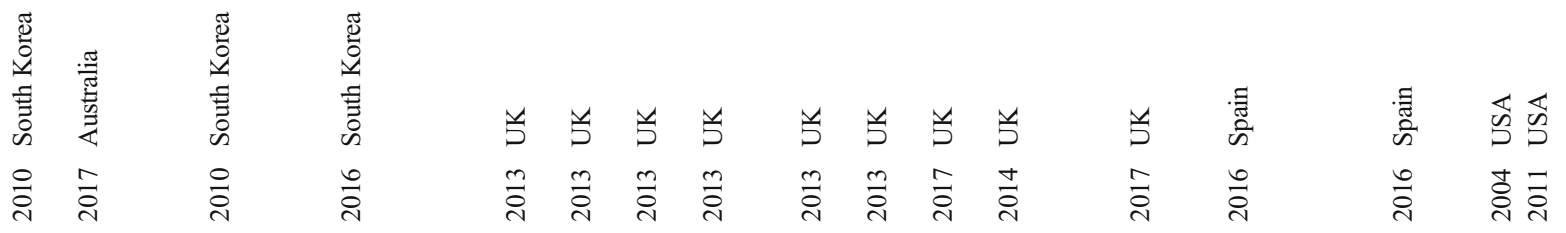
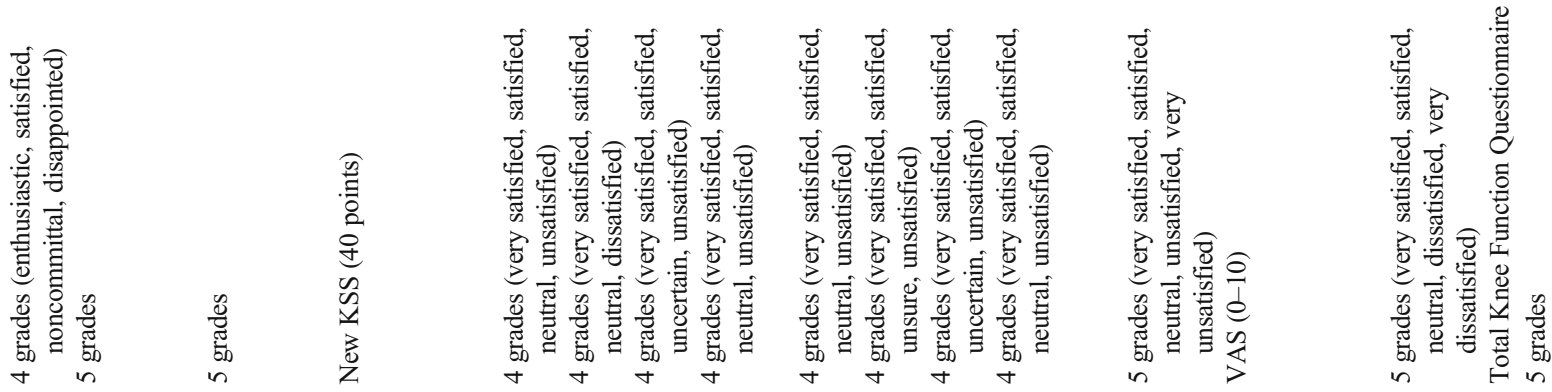

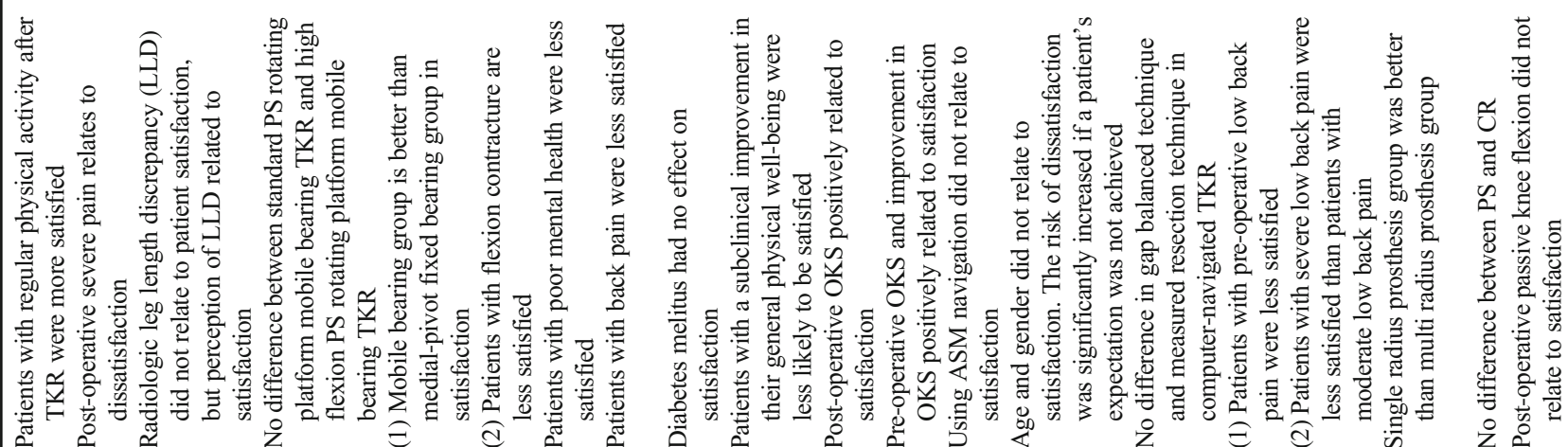




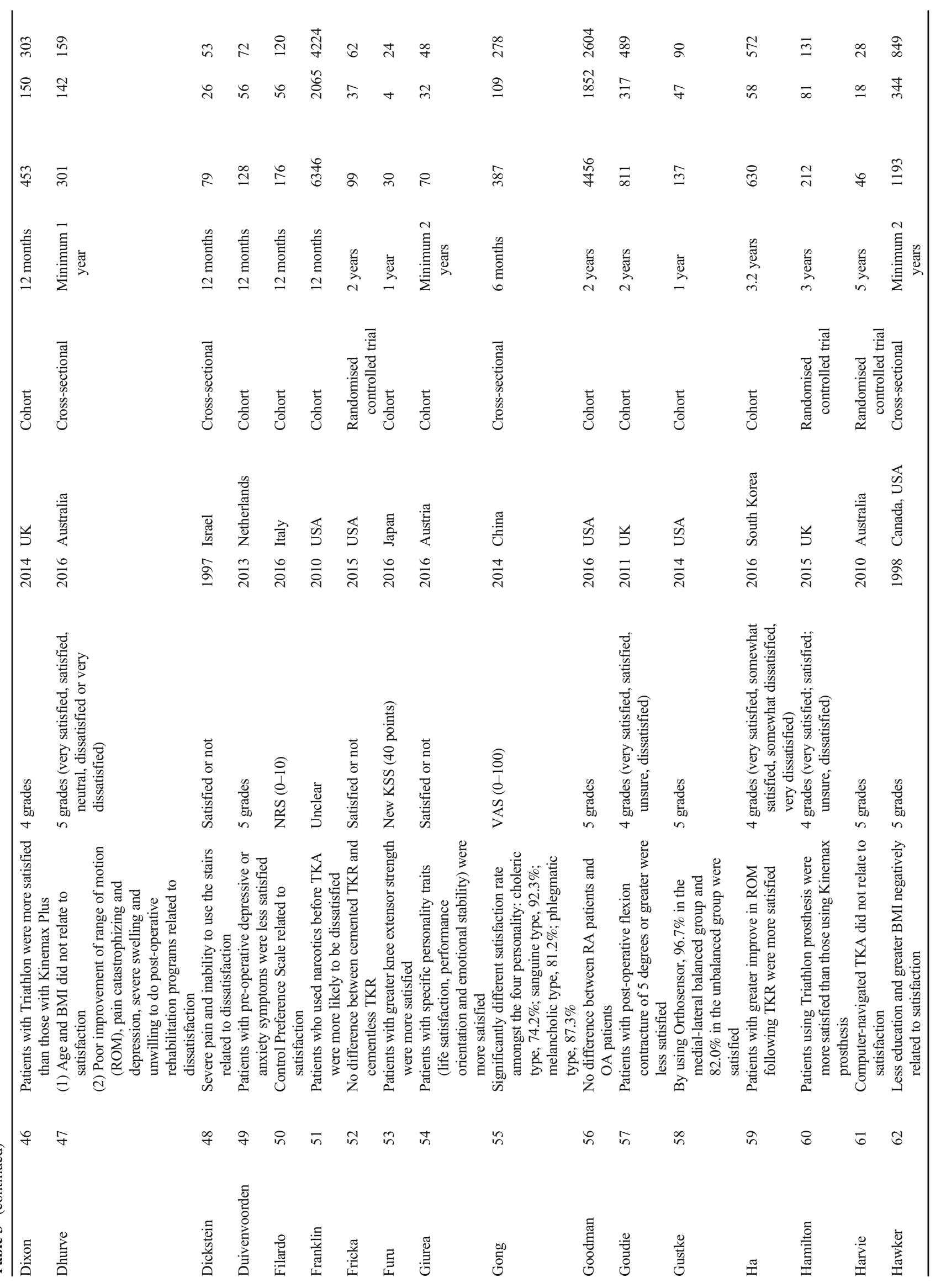




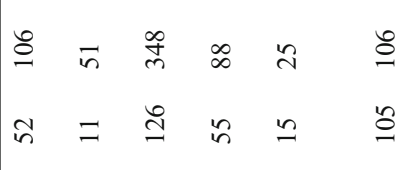

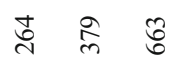
$\vec{n}$
$\stackrel{\square}{\rightarrow}$ iे $\overline{\text { nे }}$
$\circ \stackrel{\infty}{-\infty}$
究
$\bar{\sigma} \bar{i} \quad \infty \quad \infty \quad \overleftrightarrow{z}$

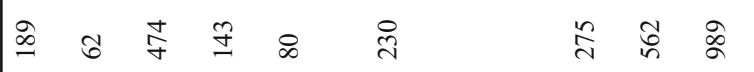
$\stackrel{\wp}{\sim}$
$\stackrel{\circ}{m}$ in के a

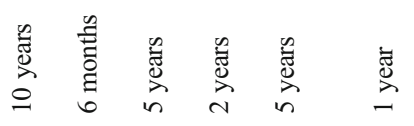

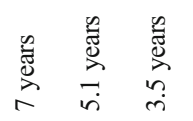

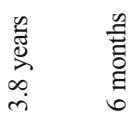

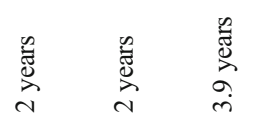

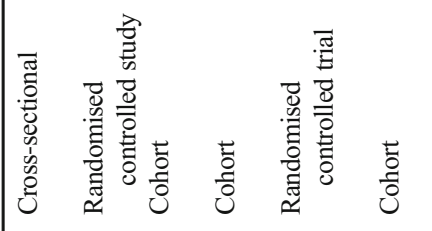

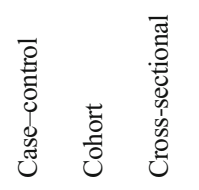

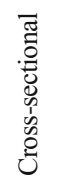

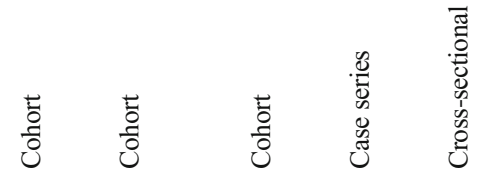

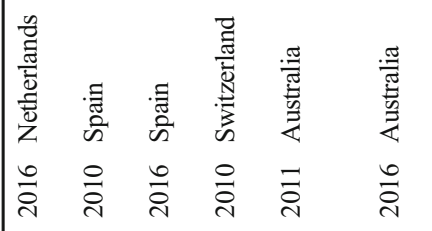

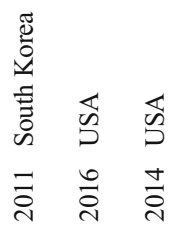

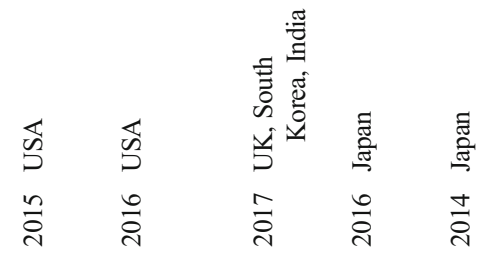

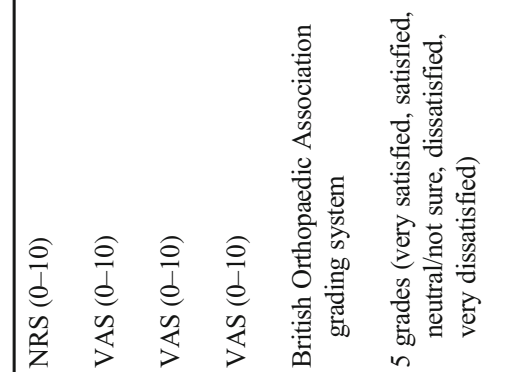

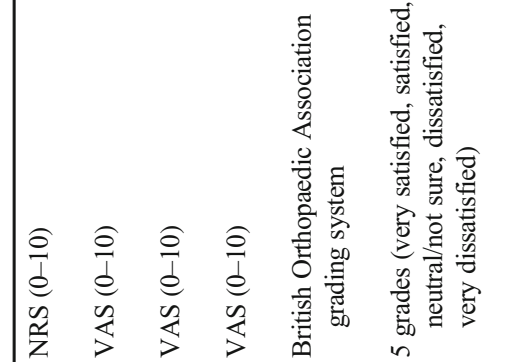

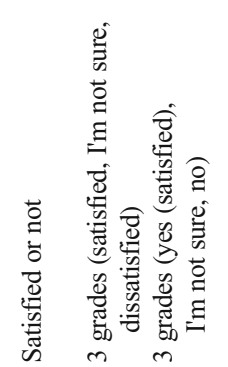

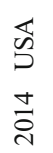

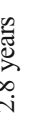
$\dot{r} 6$
ชิ สี

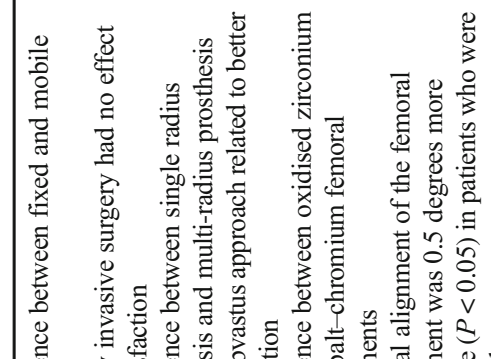

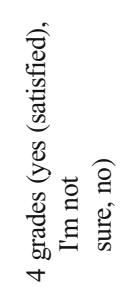

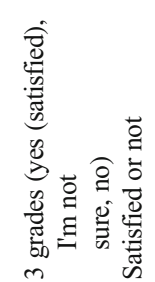

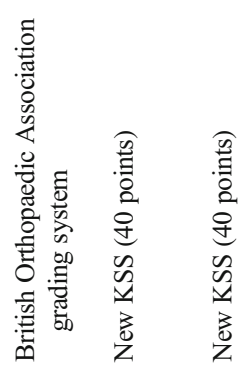

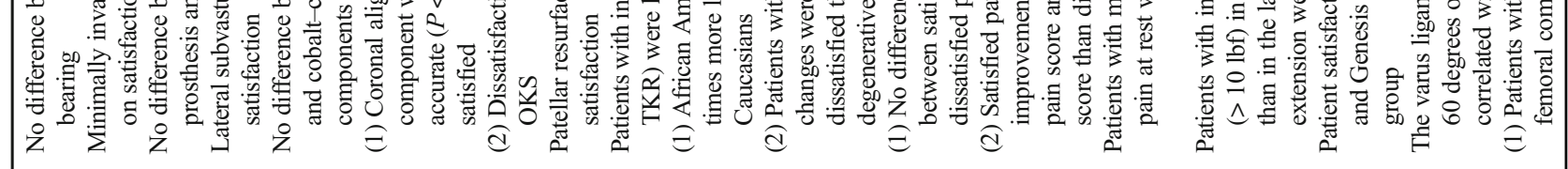

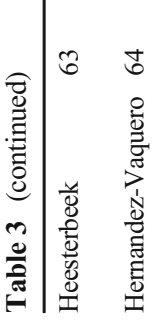
$68 \infty$
$8 ? \pi$
a
$\sqrt{2}$
总 节

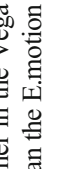

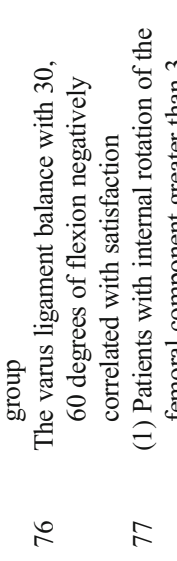

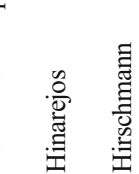

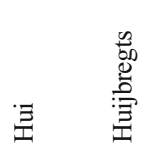

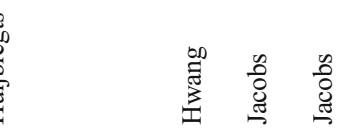
$\frac{0}{0}$
$\begin{array}{lll}n & \\ 0 & 0 \\ 0 & 0 \\ 0 & 0 \\ 0 & 0\end{array}$

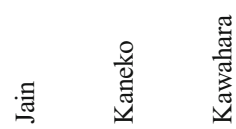




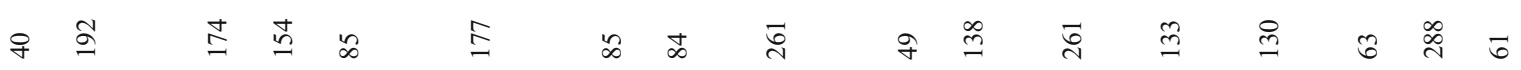

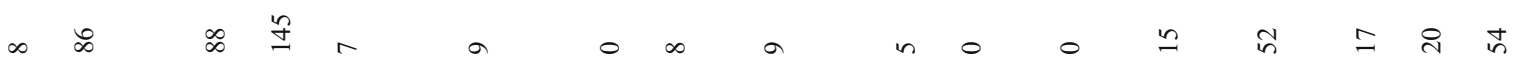

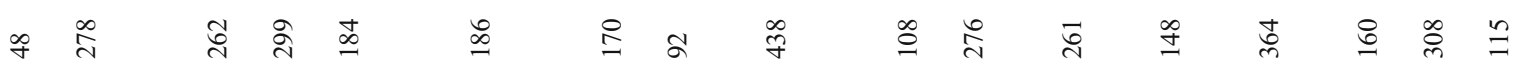

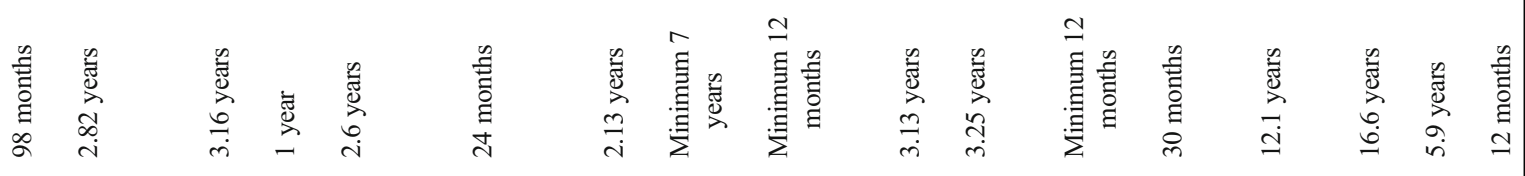

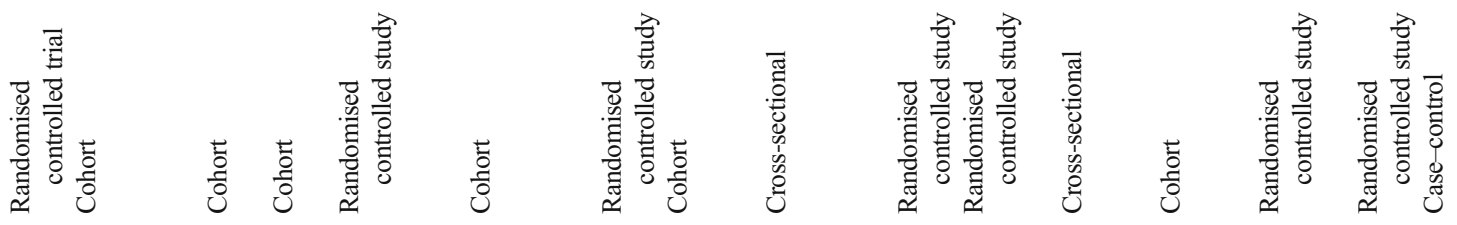

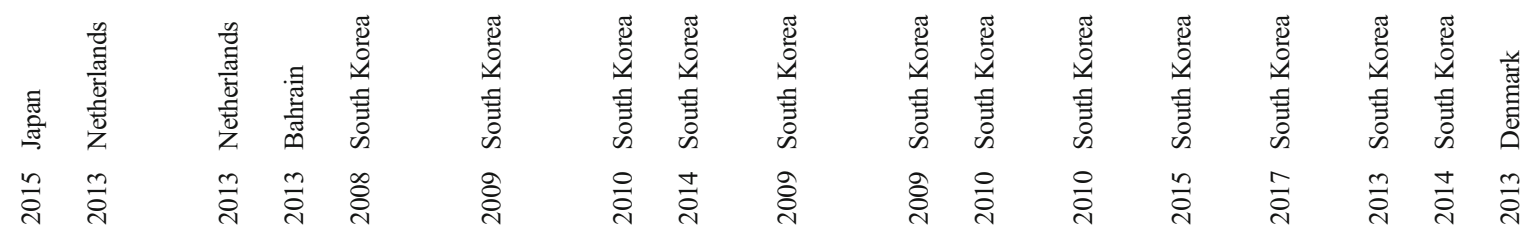

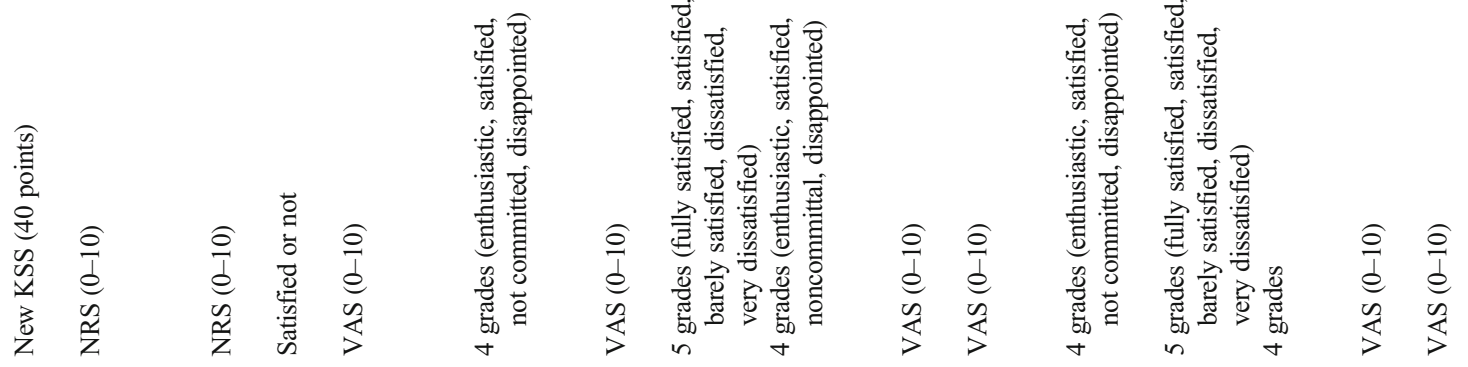

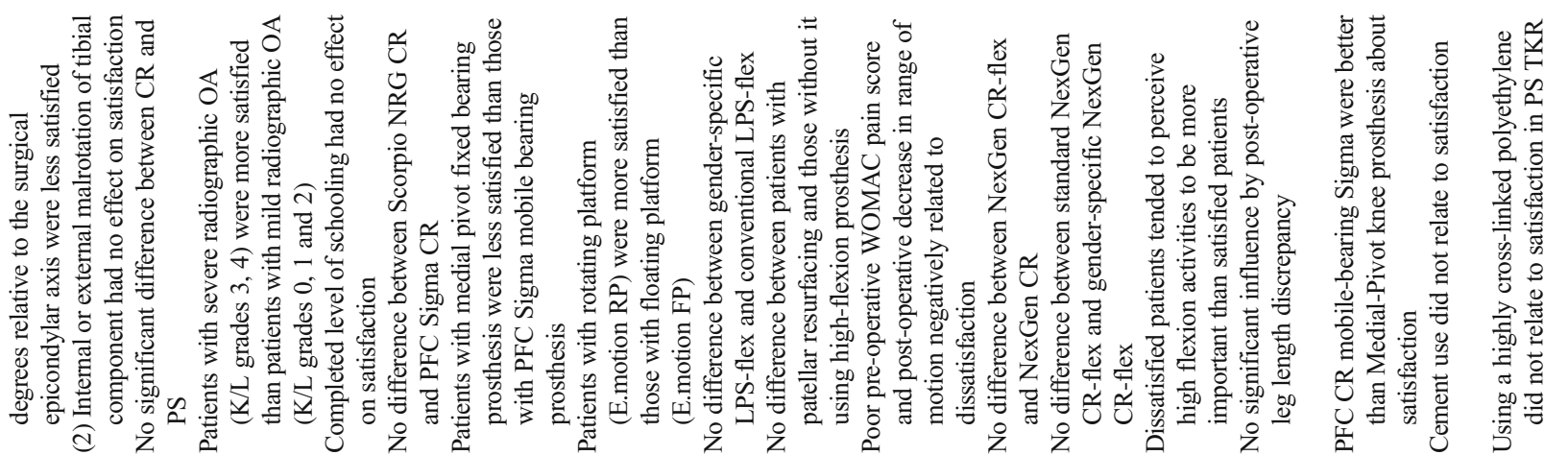

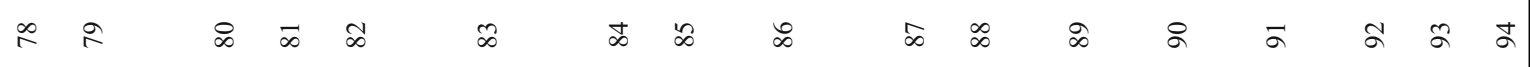

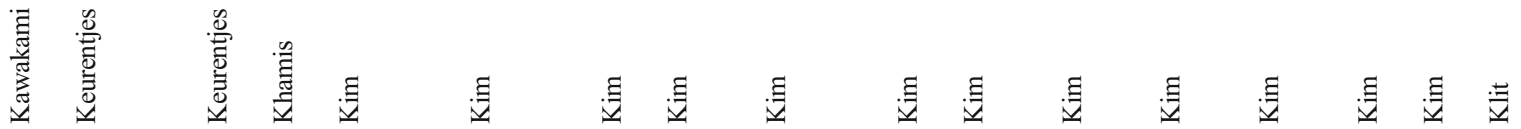




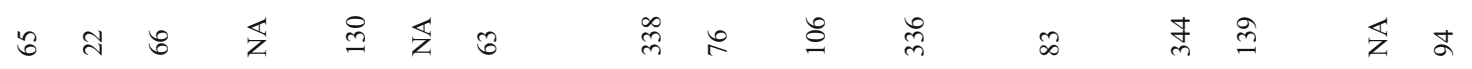

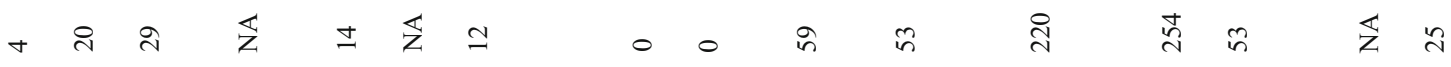

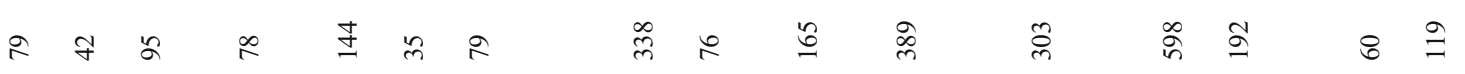

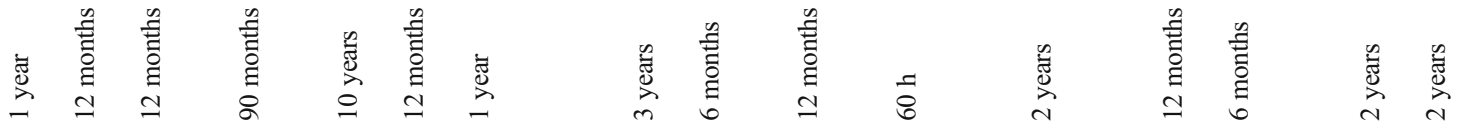

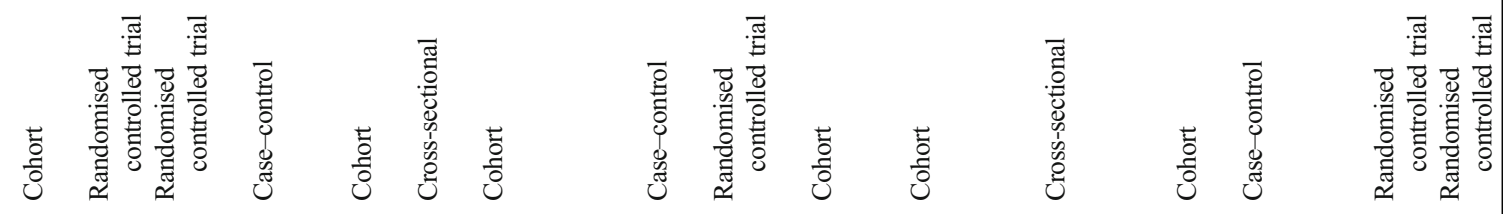

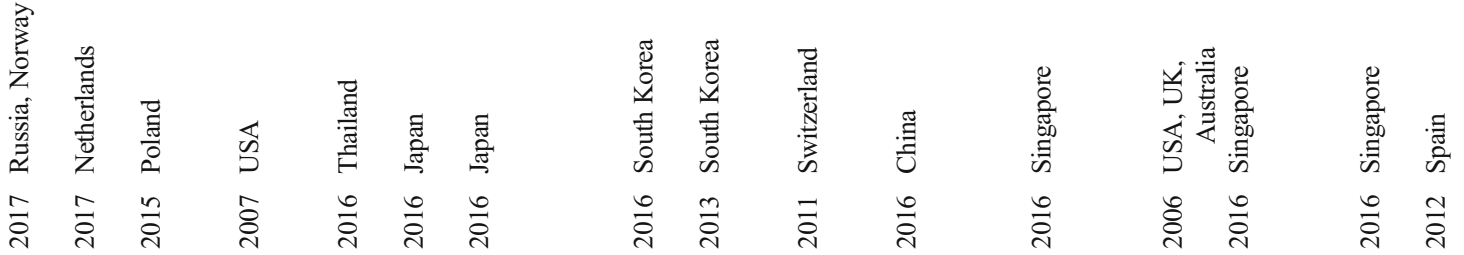

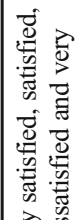

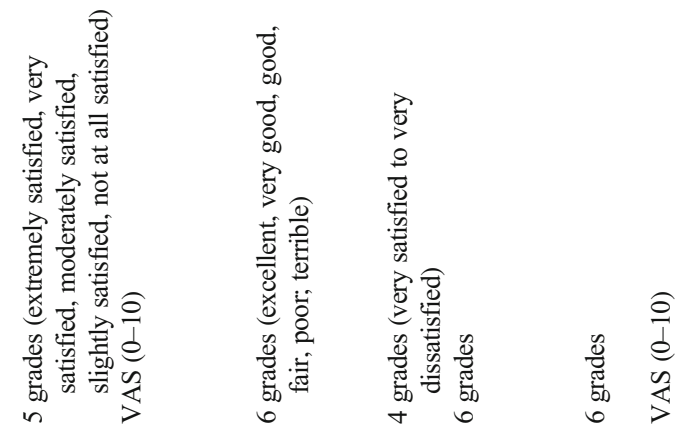

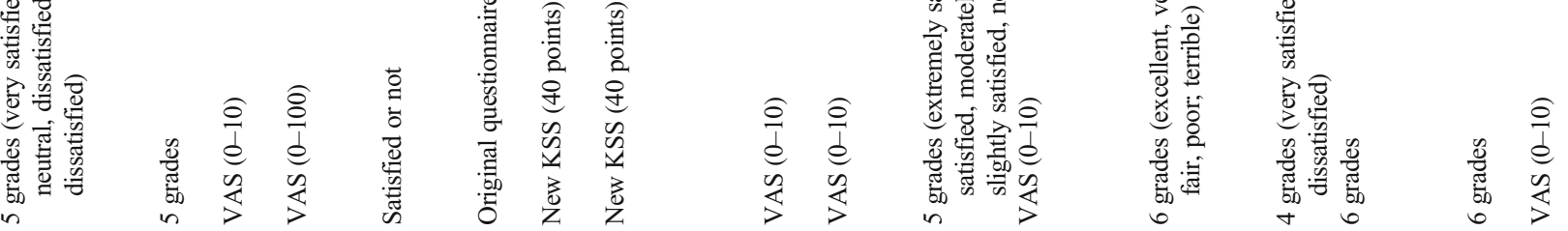

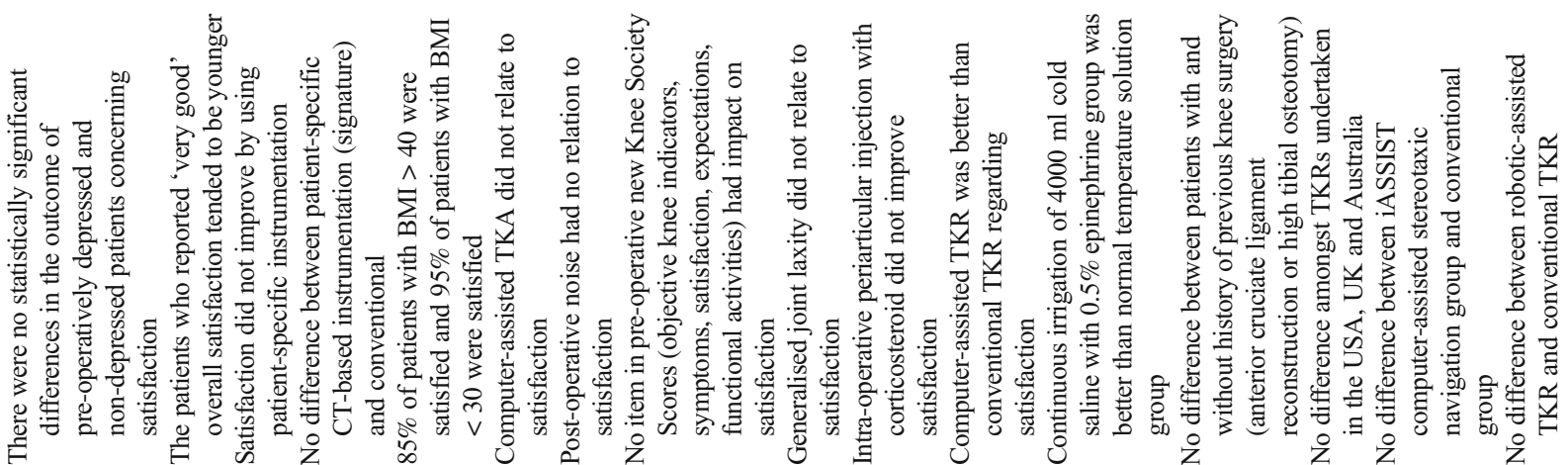

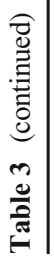
๙

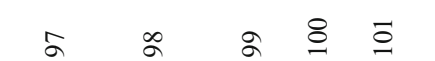
$\stackrel{\varrho}{\varrho} \stackrel{\Xi}{\varrho}$

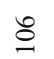
용

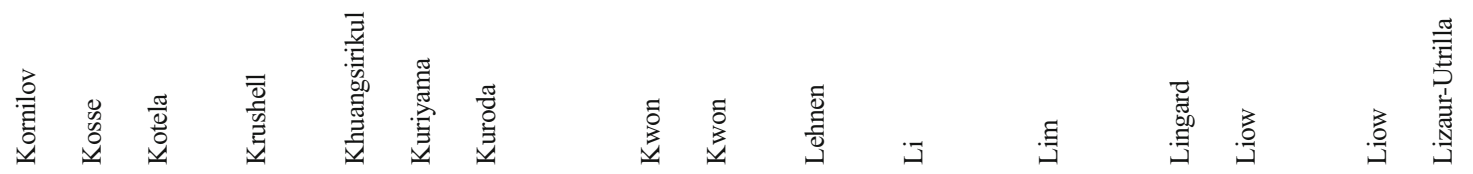




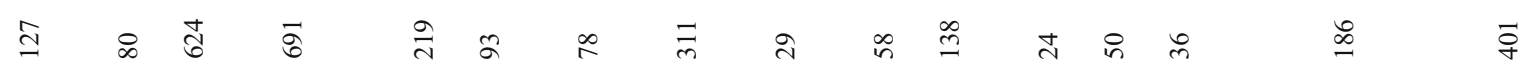

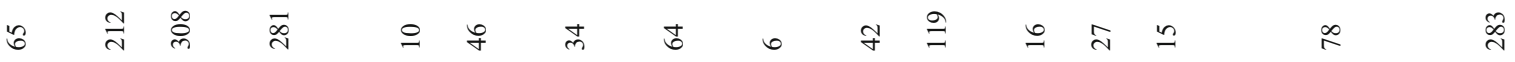

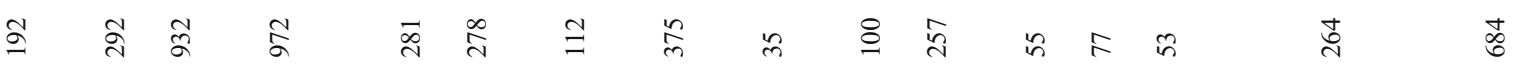

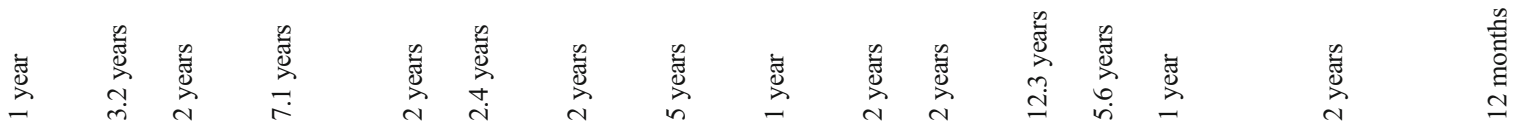

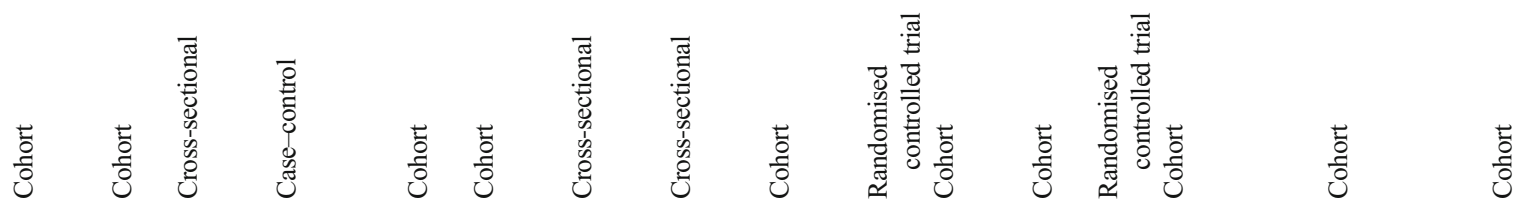

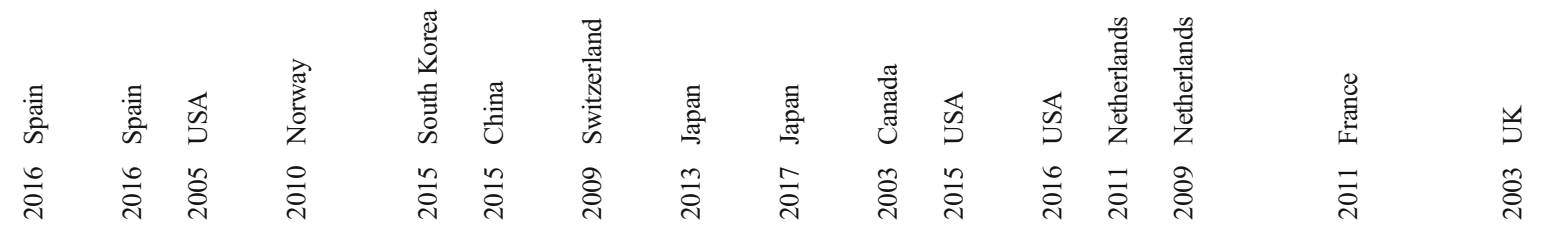
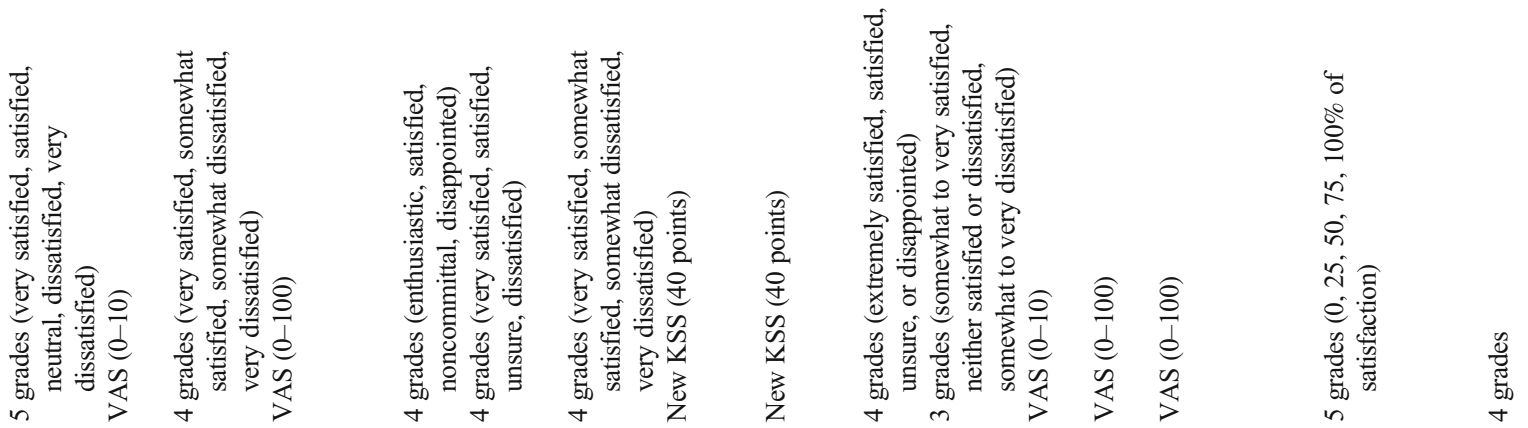

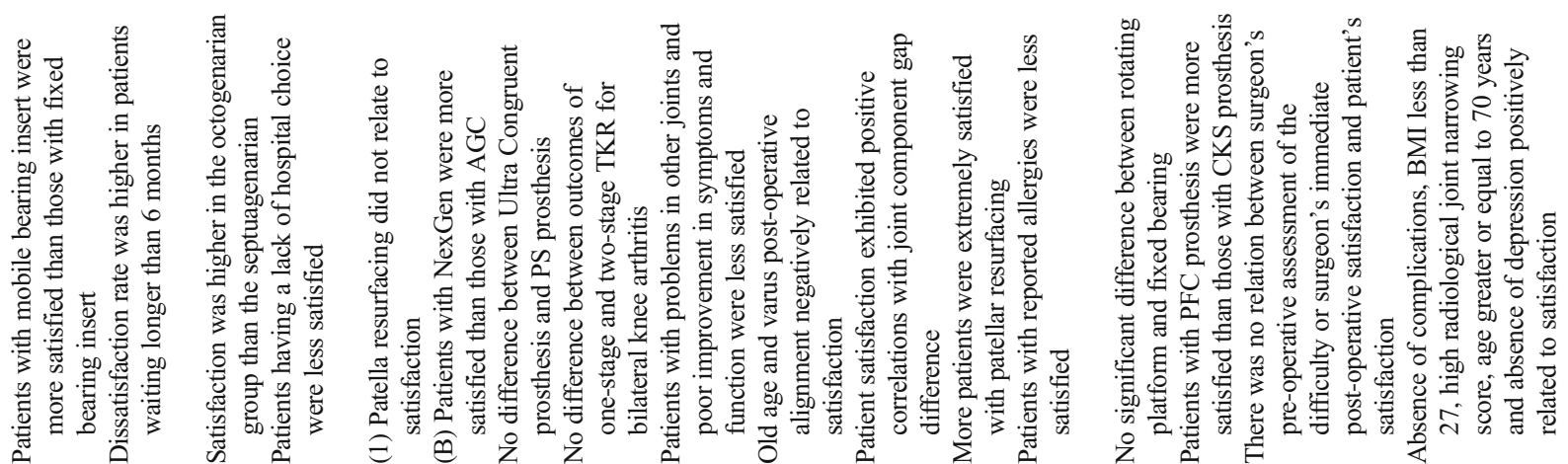

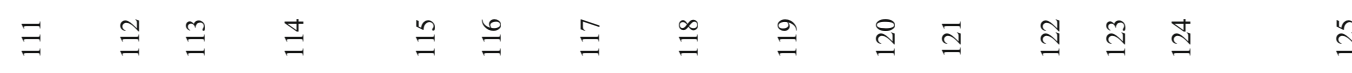

$\stackrel{ }{\beth}$

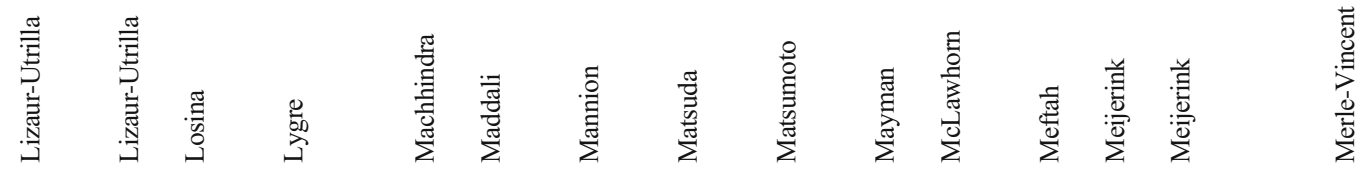

离 


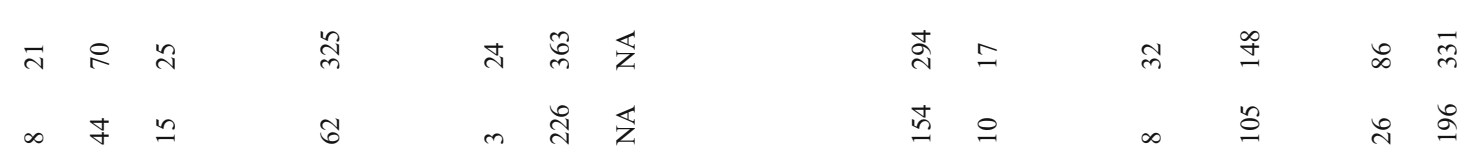

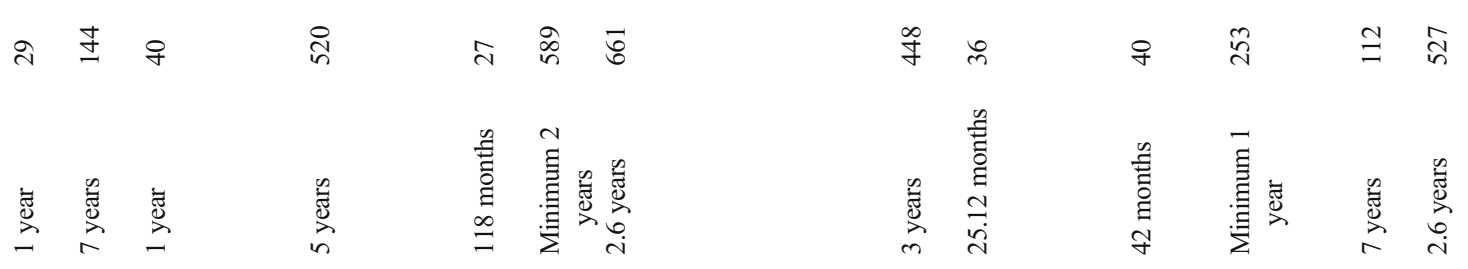

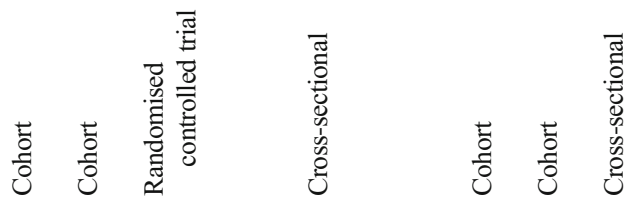

$\frac{5}{0} \frac{1}{0}$

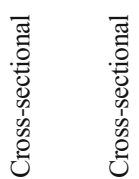

$\frac{1}{0}$
$\frac{0}{0}$

胥

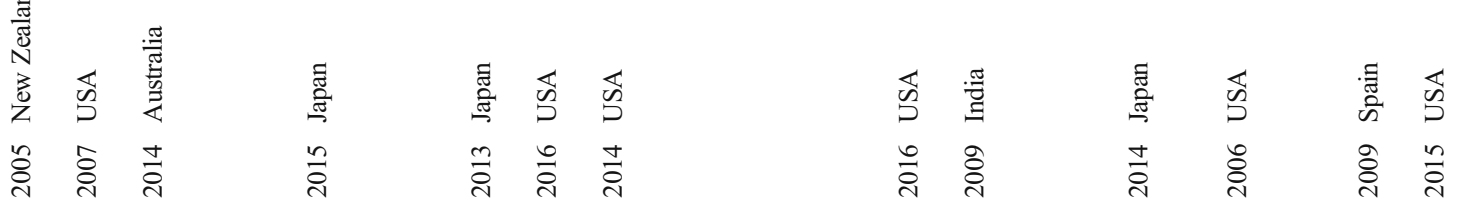

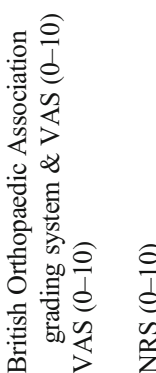

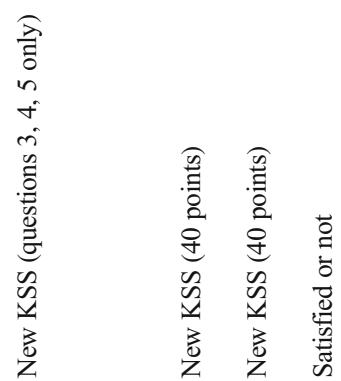

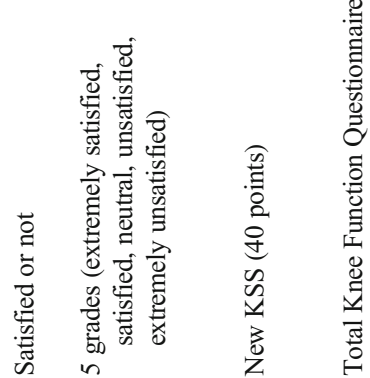

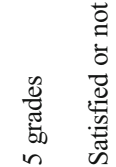

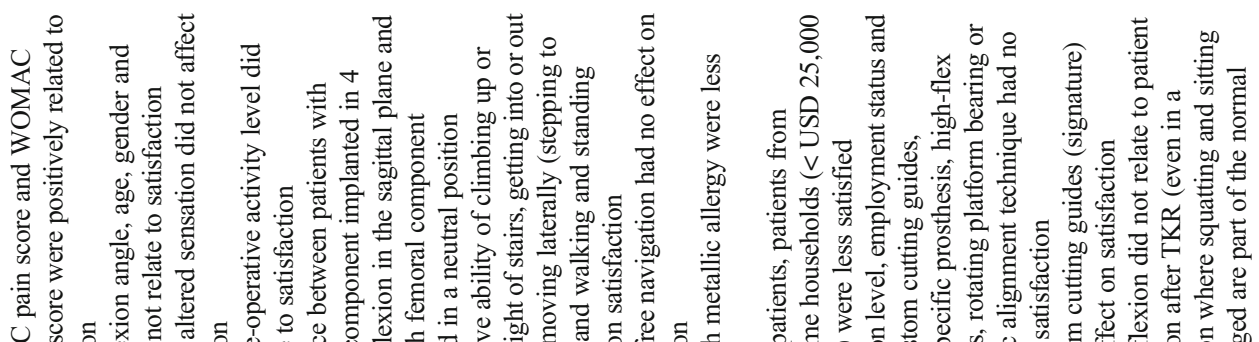

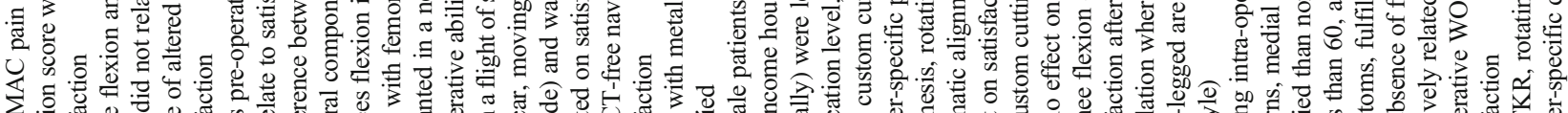

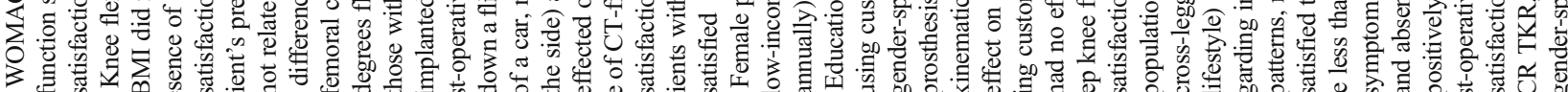

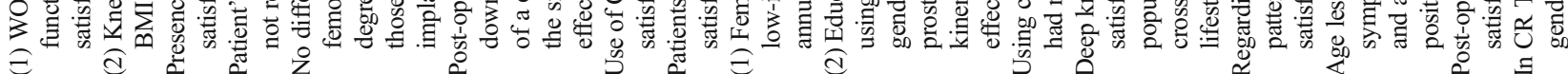

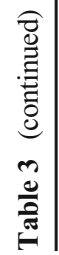
I $\stackrel{\infty}{\beth} \cong$

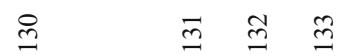
$\stackrel{\square}{2}$

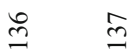
$\stackrel{\infty}{\dddot{m}}$

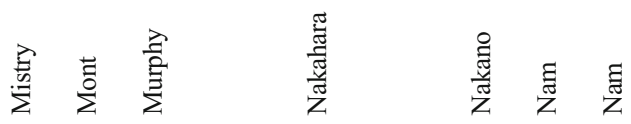
宽
产
害 竞 


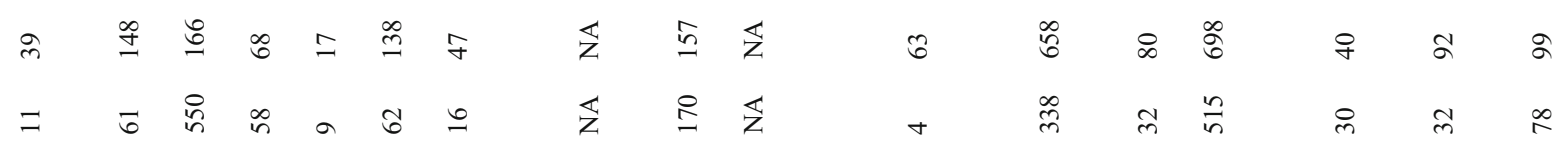

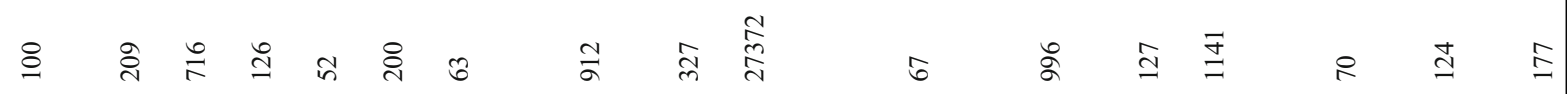

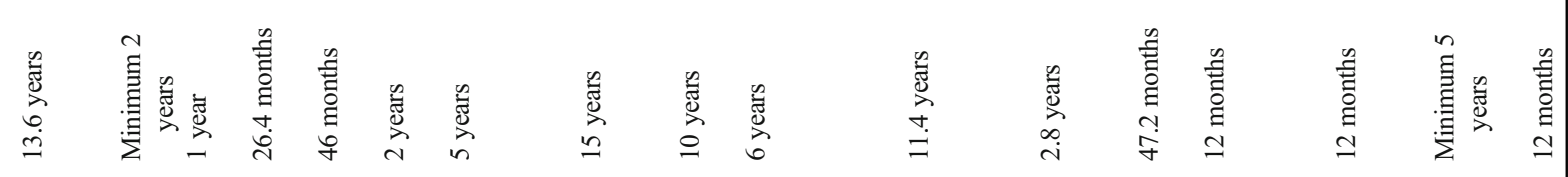

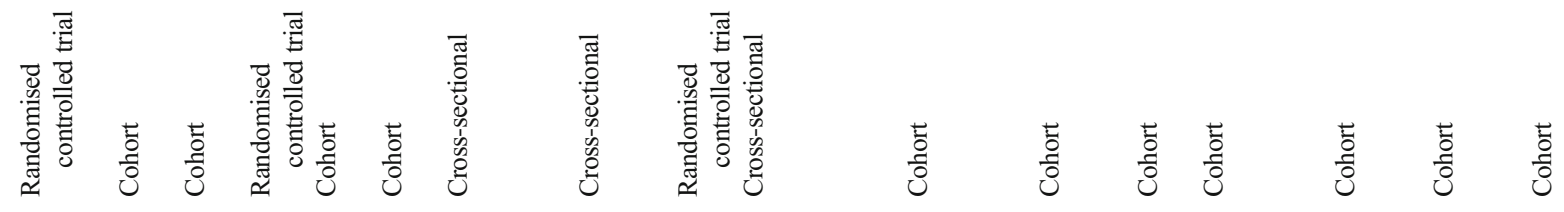

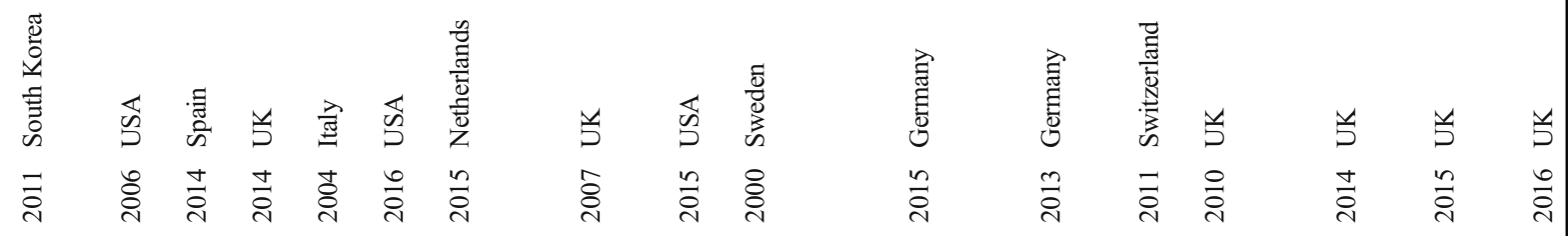

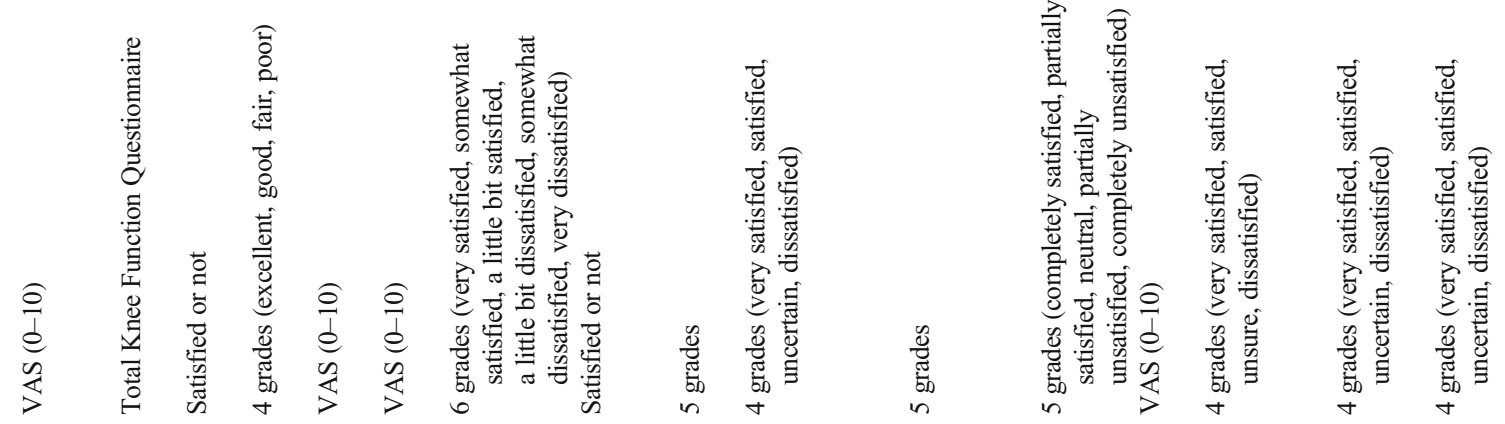

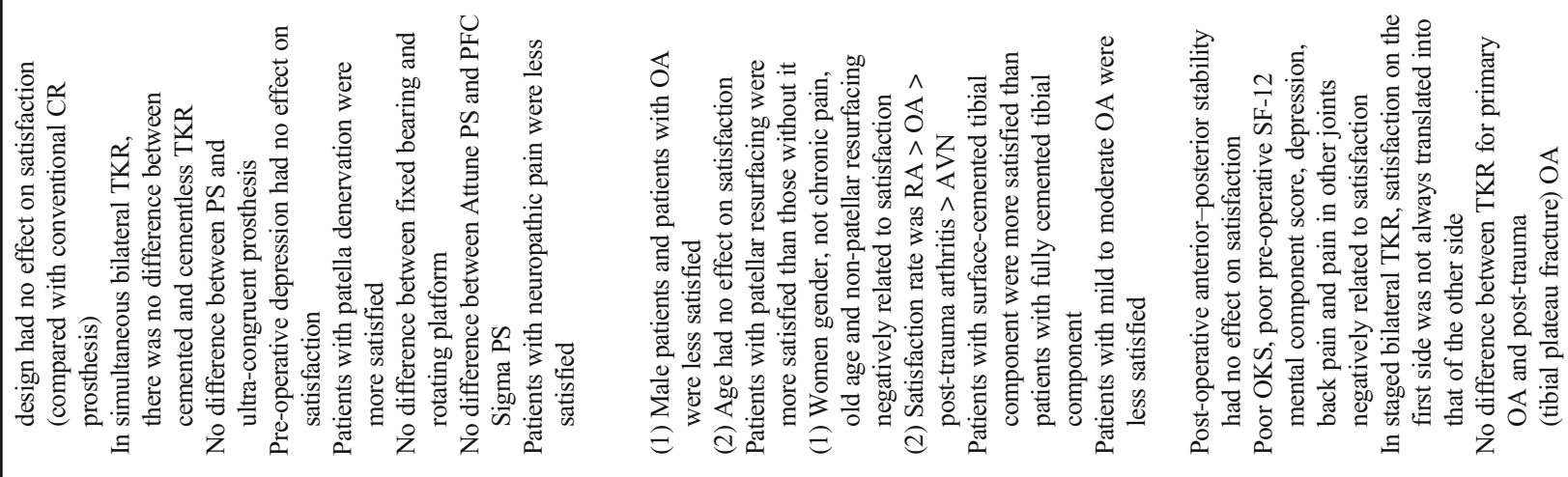

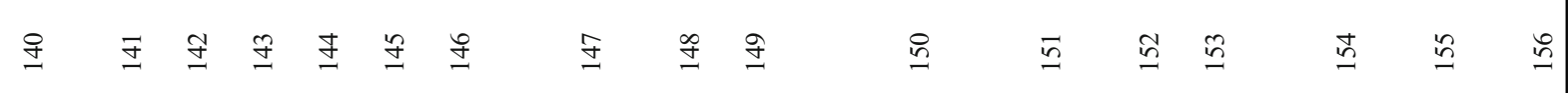

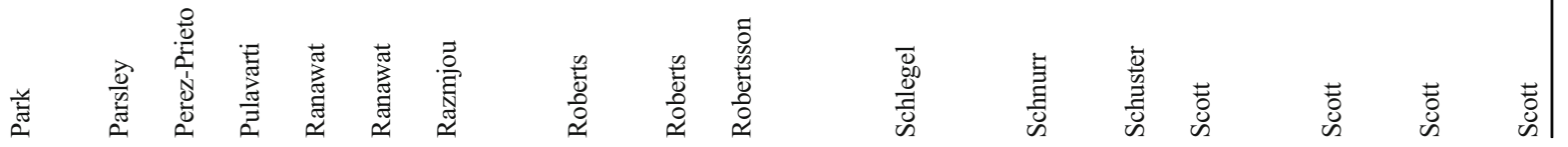




\begin{tabular}{|c|c|c|c|c|c|c|c|c|c|c|c|c|}
\hline ป & ळे & $\ddot{n}$ & i & ัે & $\hat{\beta}$ & 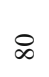 & $\stackrel{\infty}{\infty}$ & 2 & $\dot{d} \bar{m}$ & $\approx \widetilde{z}$ & f & $\stackrel{d}{d}$ \\
\hline$\infty$ & $\infty$ & $\stackrel{J}{\sim}$ & สี & $\bar{n}$ & $\underset{m}{t}$ & $\mathbb{2}$ & तี & \pm & 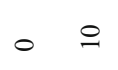 & $\leadsto \mathbb{z}$ & $\because$ & ㄱ. \\
\hline e & in & g & $\approx$ & $\tilde{n}$ & $\bar{\Xi}$ & $\cong$ & 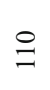 & 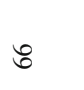 & $\stackrel{\infty}{+}$ & $\stackrel{\infty}{q}$ & 8 & Ұ \\
\hline 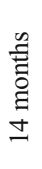 & $\underset{\stackrel{\Xi}{\beth}}{\stackrel{\vec{\Xi}}{二}}$ & 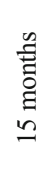 & 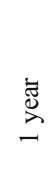 & 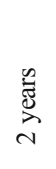 & $\stackrel{\vec{J}}{\stackrel{\vec{J}}{二}}$ & 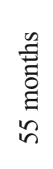 & $\stackrel{\Xi}{\stackrel{\Xi}{\Xi}}$ & $\underset{\gtrsim}{\stackrel{\varpi}{\varpi}}$ & 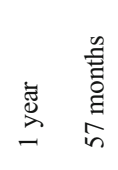 & 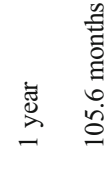 & 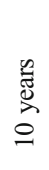 & $\begin{array}{l}\text { 气 } \\
\overline{\bar{E}} \\
\text { हू }\end{array}$ \\
\hline to & $\begin{array}{l}\text { t: } \\
\frac{0}{0}\end{array}$ & 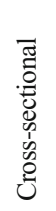 & $\begin{array}{l}\frac{\pi}{0} \\
\frac{0}{0} \\
0\end{array}$ & 总 & 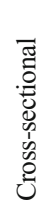 & 苟 & $\begin{array}{l}\text { t: } \\
\text { 잉 }\end{array}$ & 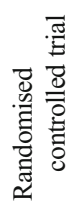 & 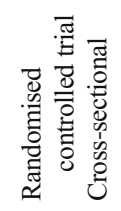 & 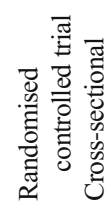 & $\begin{array}{l}5 \\
\frac{0}{0} \\
0\end{array}$ & 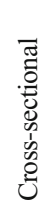 \\
\hline
\end{tabular}

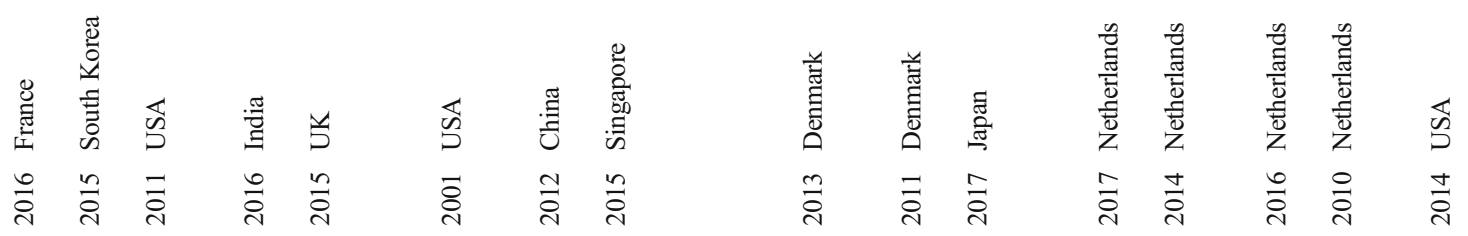

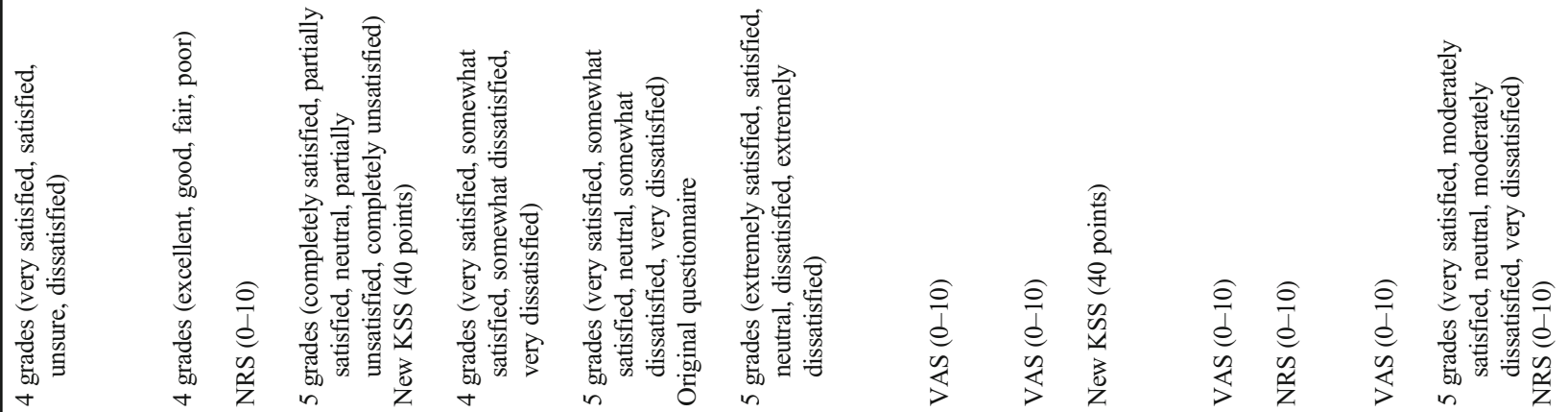

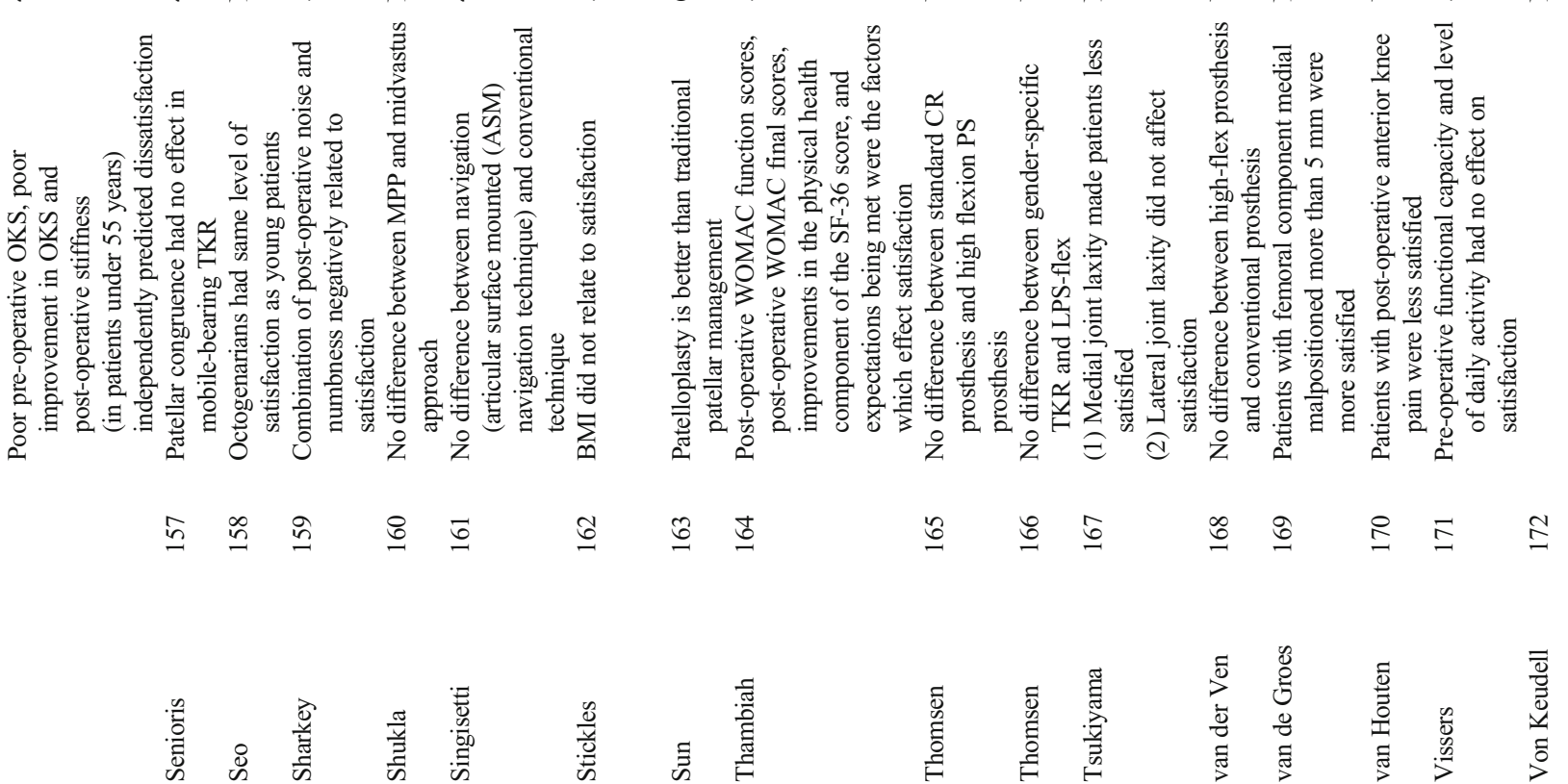




\begin{tabular}{|c|c|c|c|}
\hline $\mathbb{z} \quad \tilde{d} \quad g$ & $\frac{t}{m}$ & & a \\
\hline in $\bar{m}$ & $\underline{I}$ & $\cong$ & + \\
\hline
\end{tabular}

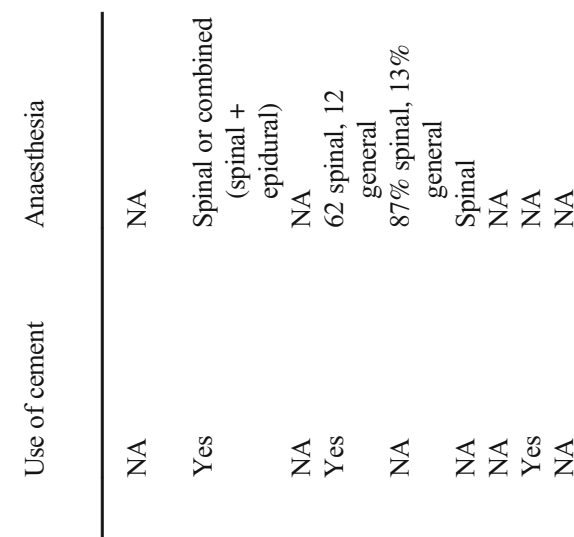

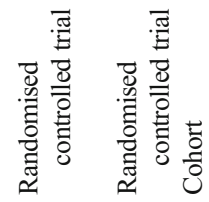
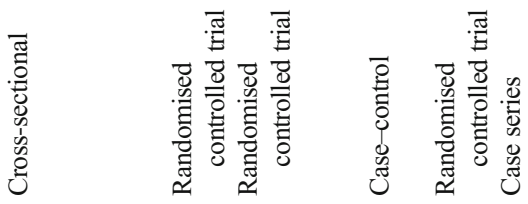

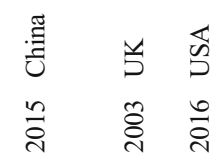
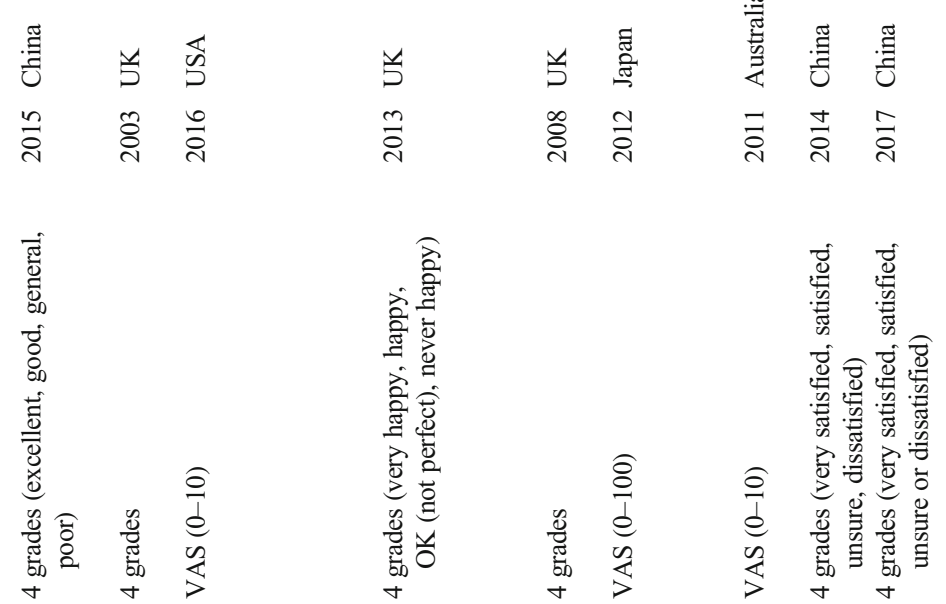

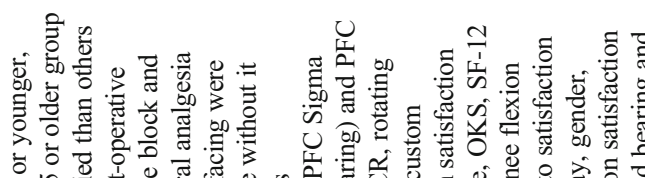

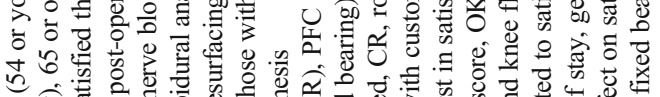

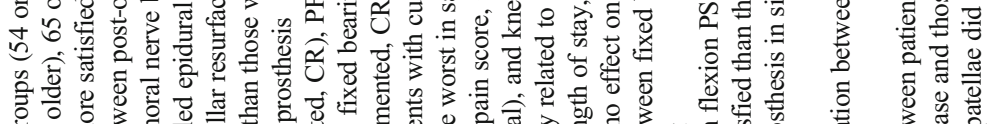

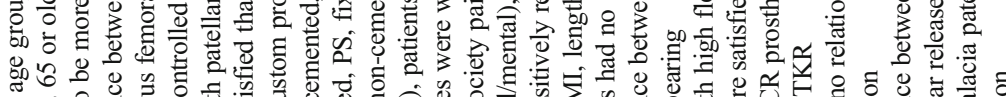

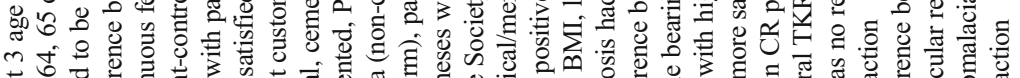

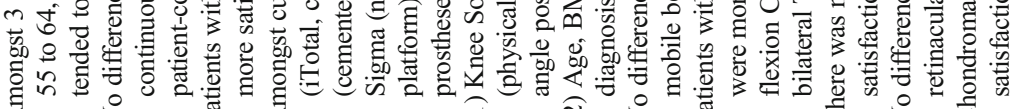

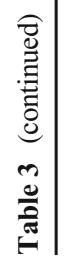

$\stackrel{5}{n}$

$\stackrel{ }{\cong} \stackrel{\infty}{=}$

$\stackrel{\infty}{\triangle} \underset{\infty}{\square}$

矢

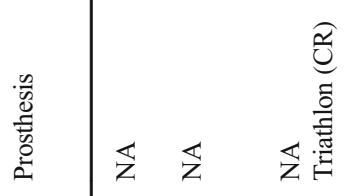

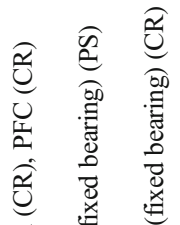

z $z E$ 焉

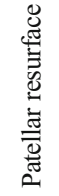

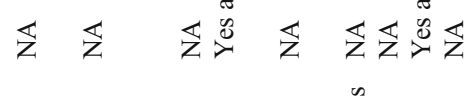

융<smiles>CCCCC</smiles>

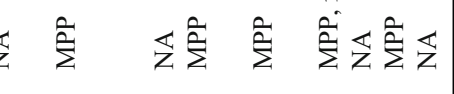

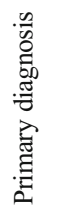

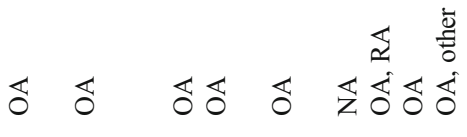

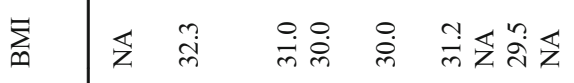

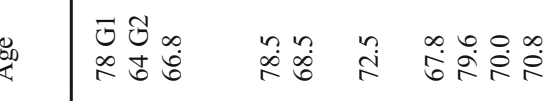

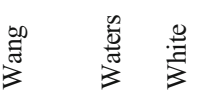

善

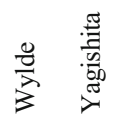

旁 $\frac{\pi}{2}$

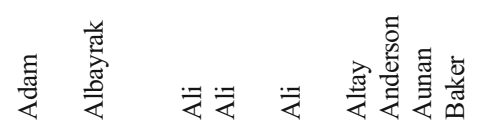




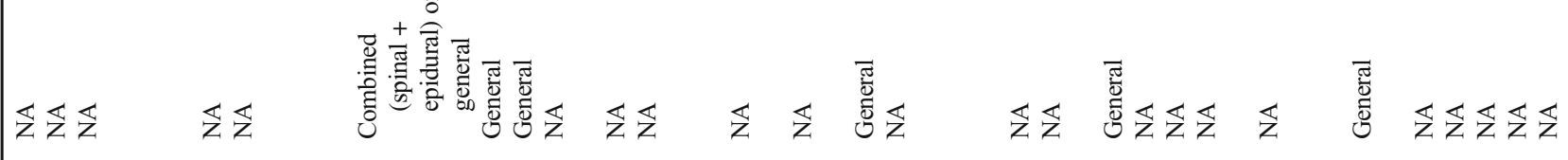

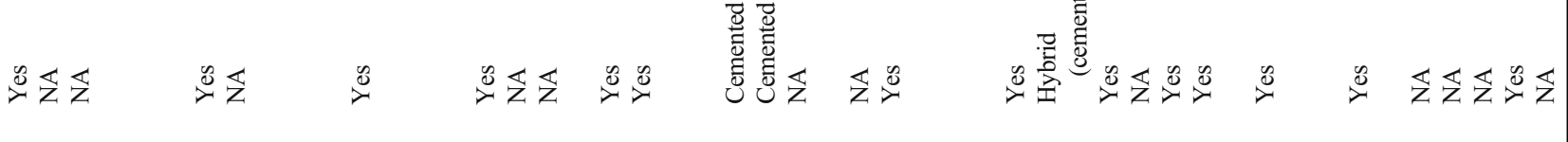

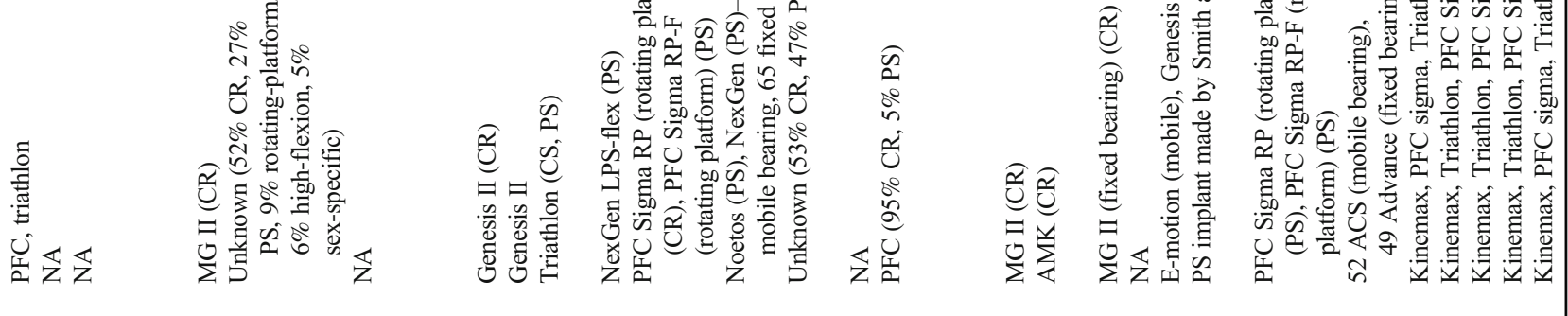

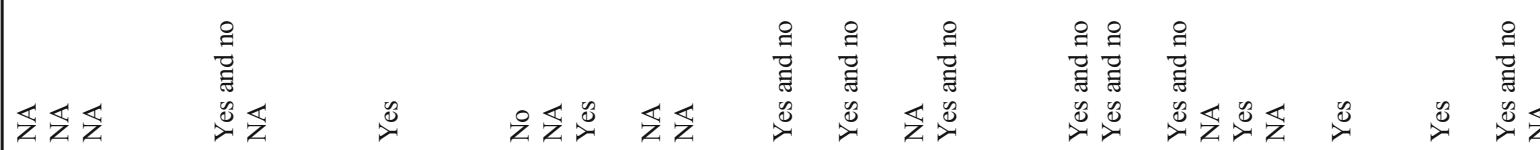
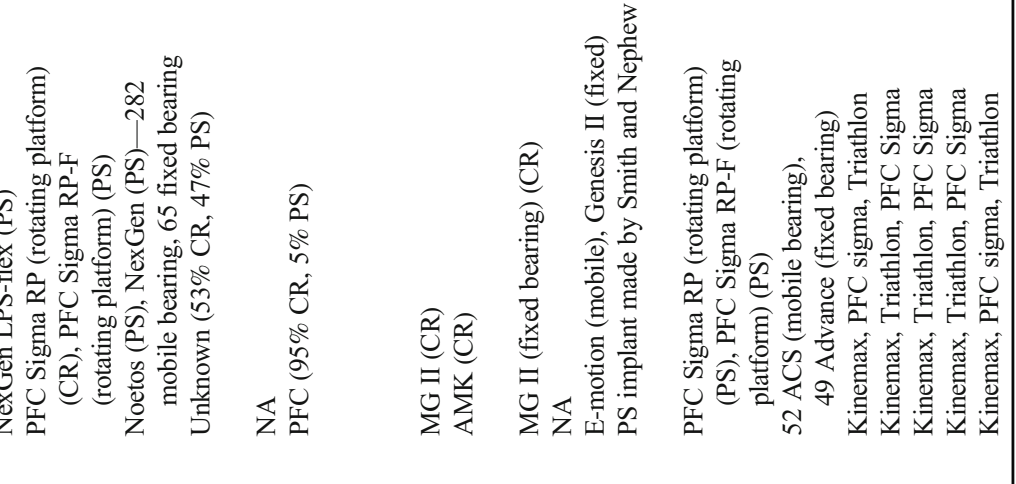
r

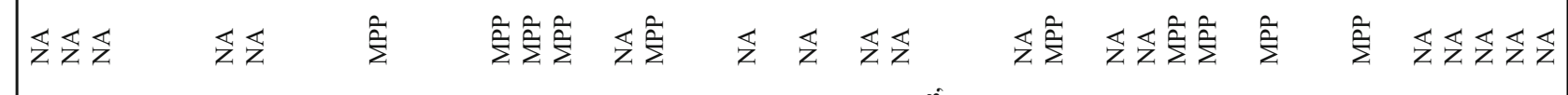

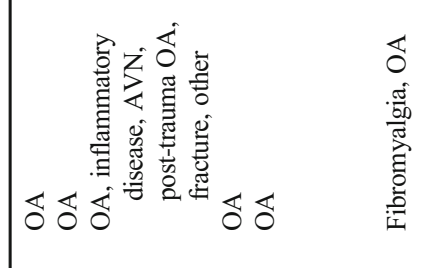

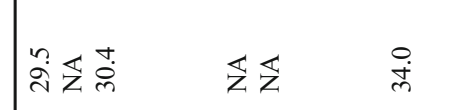

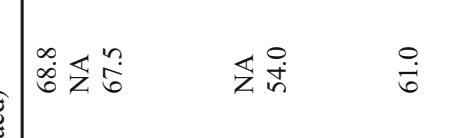

孚

$$
\text { (a) }
$$

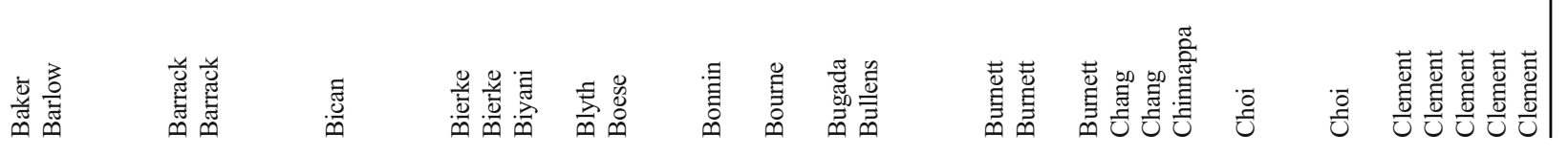




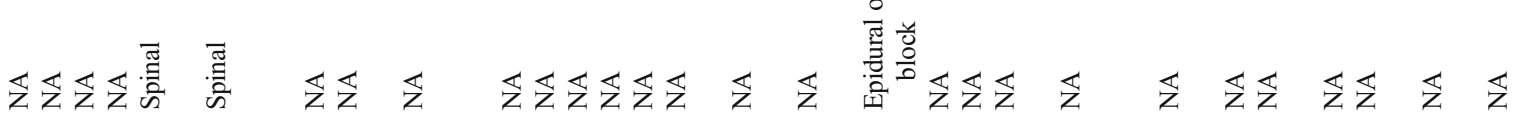

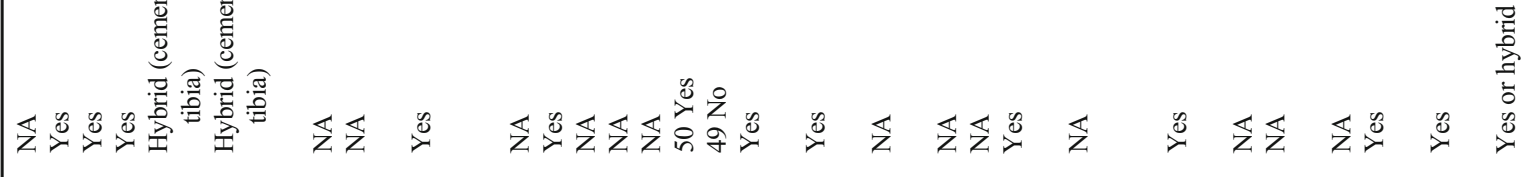

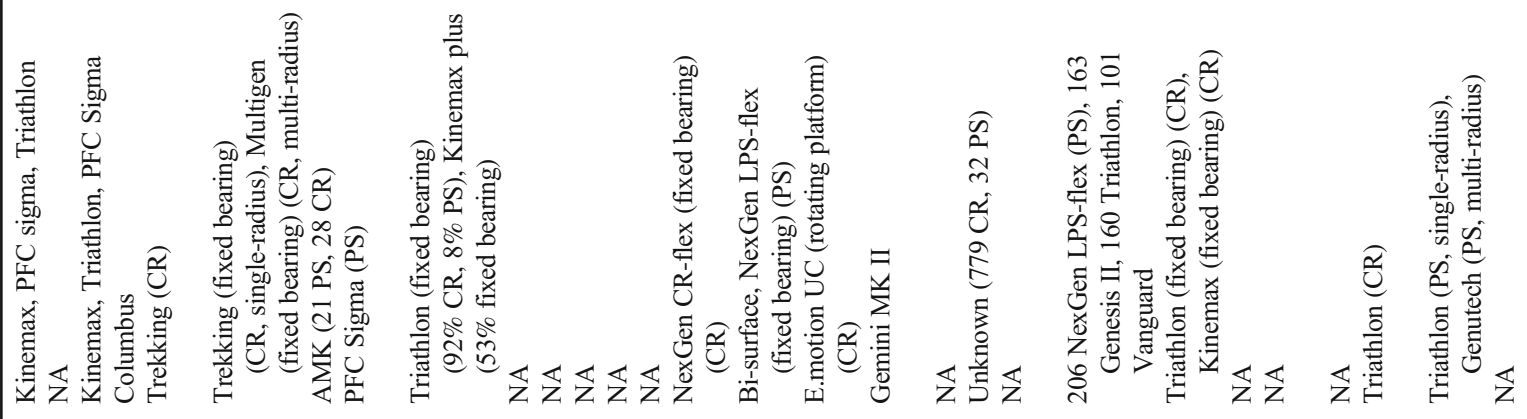

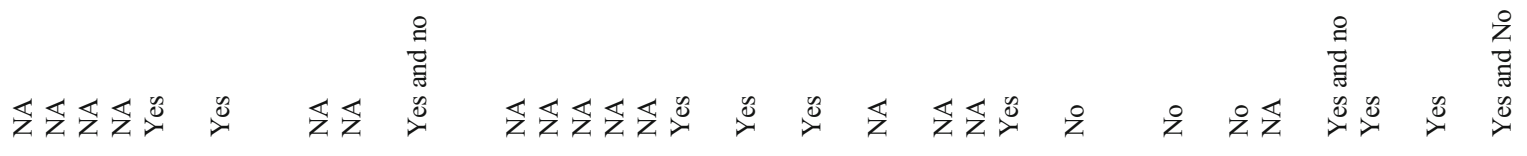

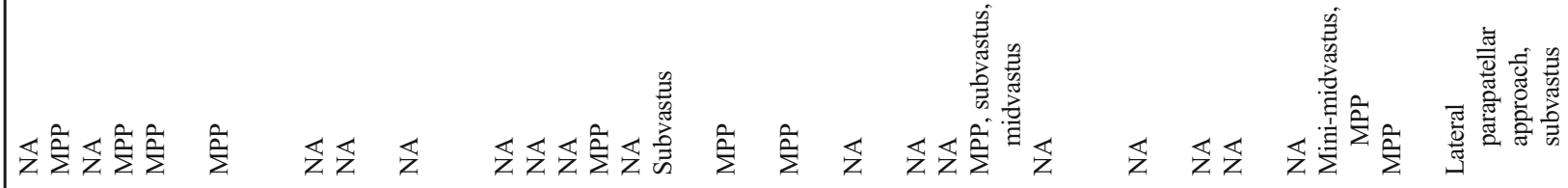

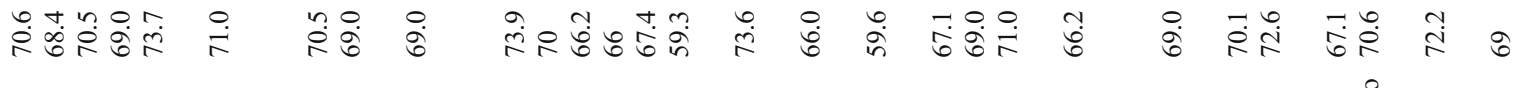

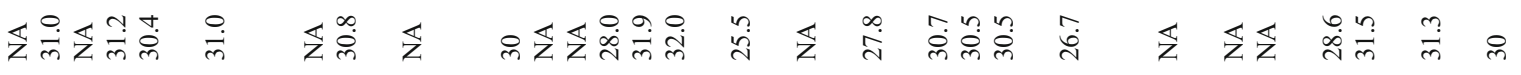

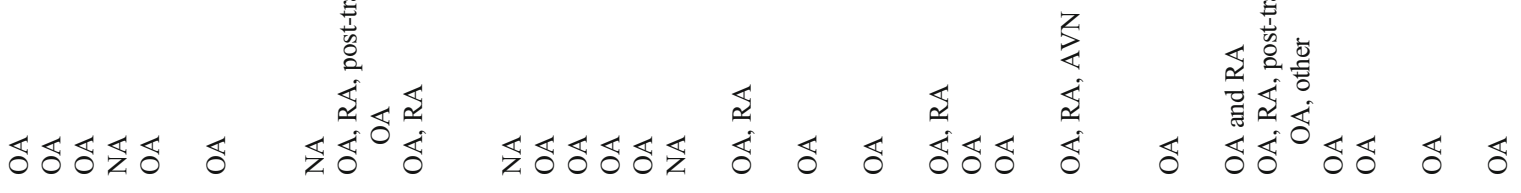<smiles>CCCC</smiles> 


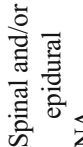

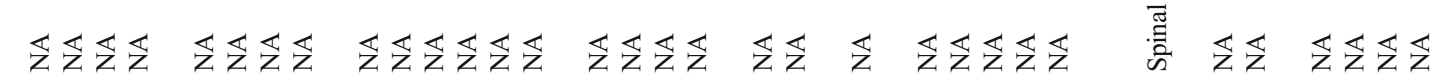

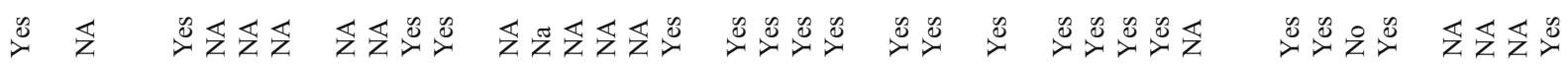

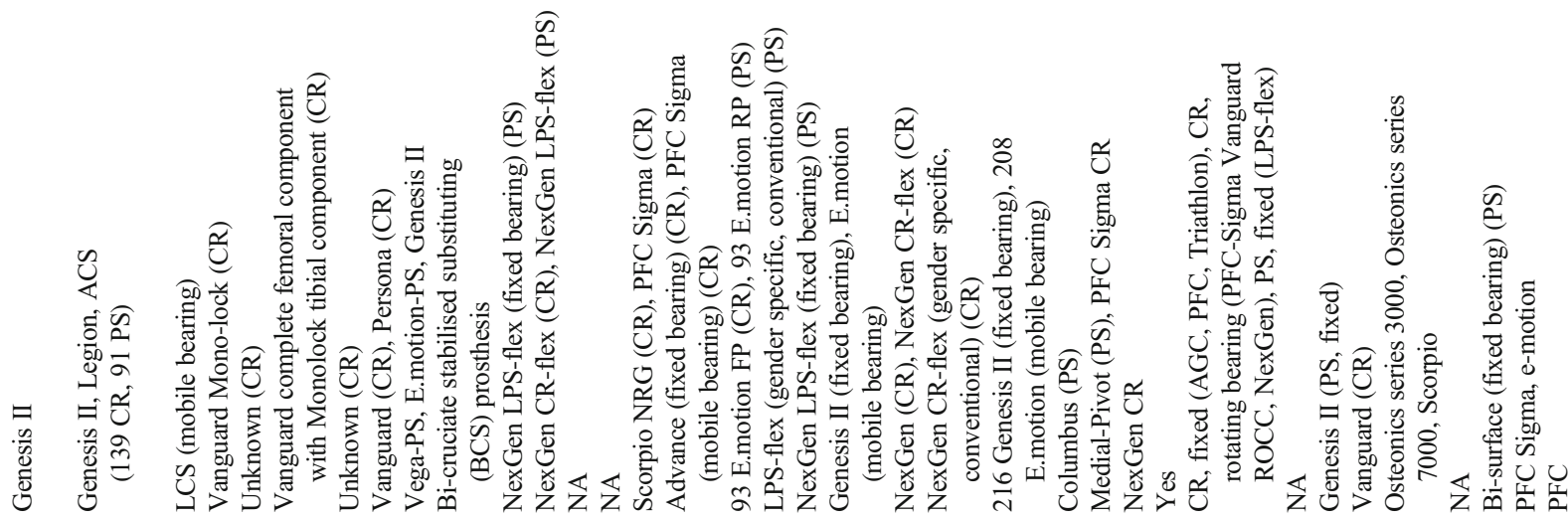

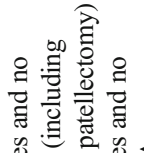

竞

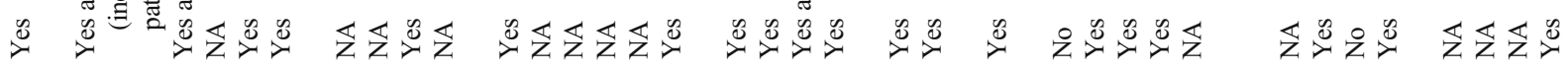

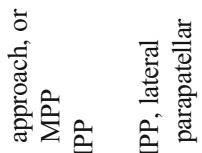

言

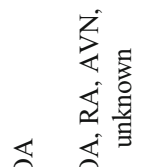

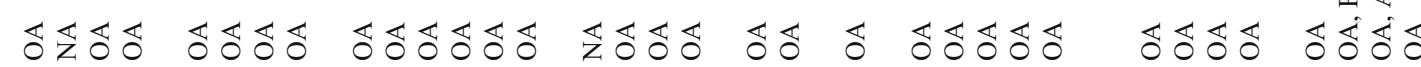

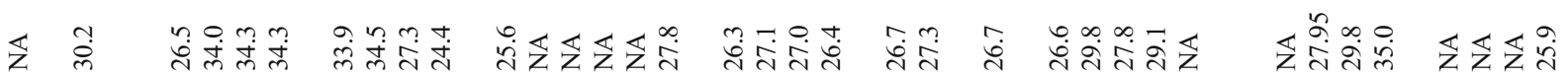

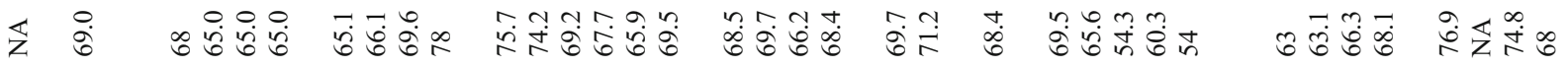

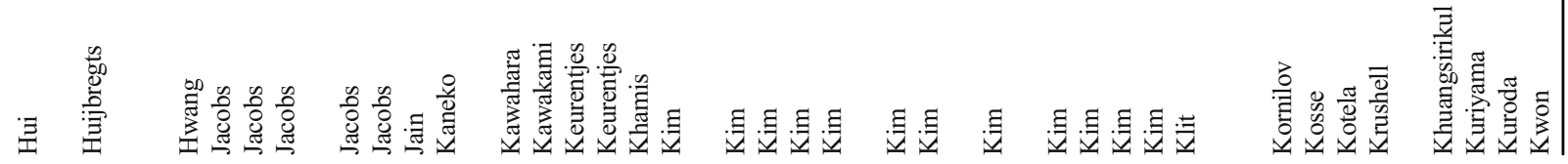




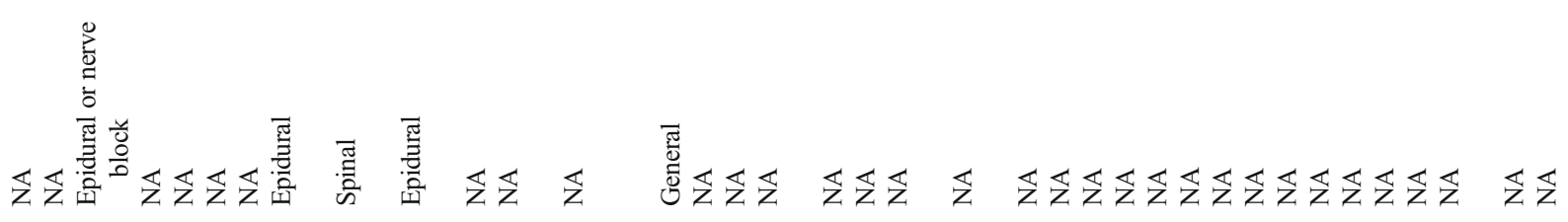

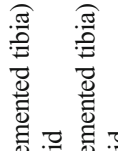

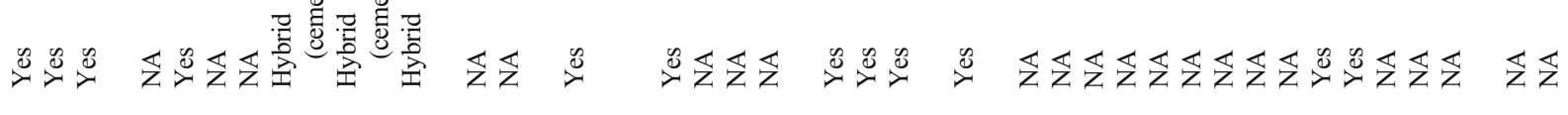

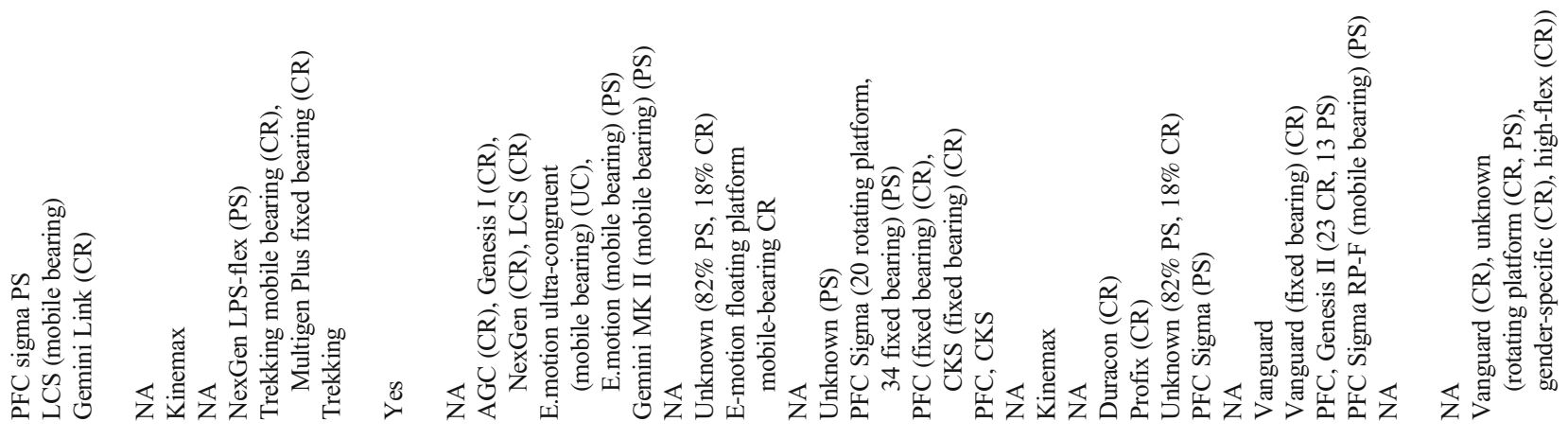

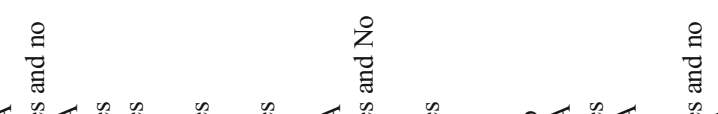

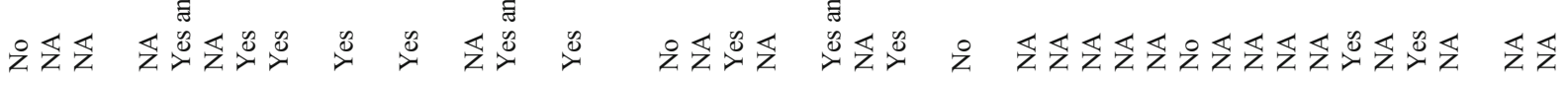

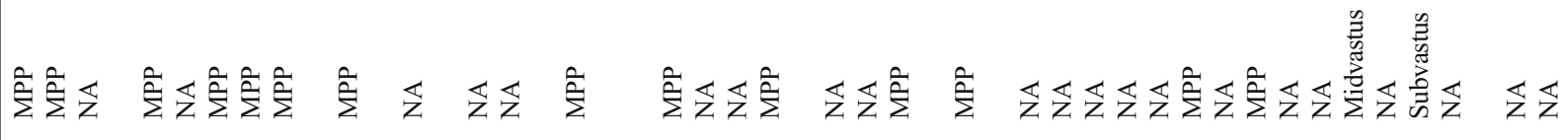

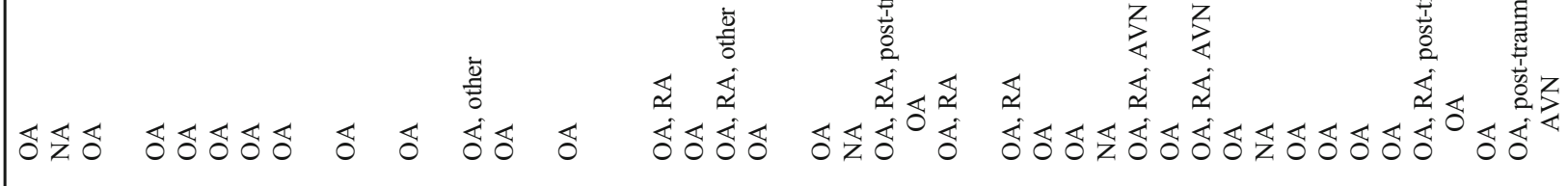

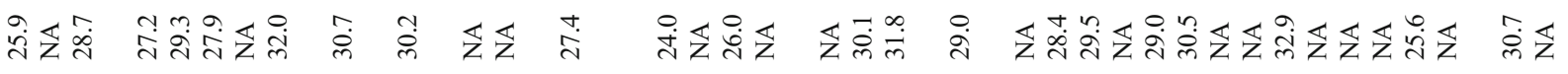

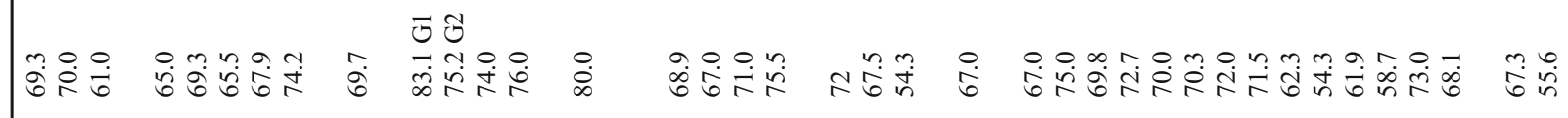

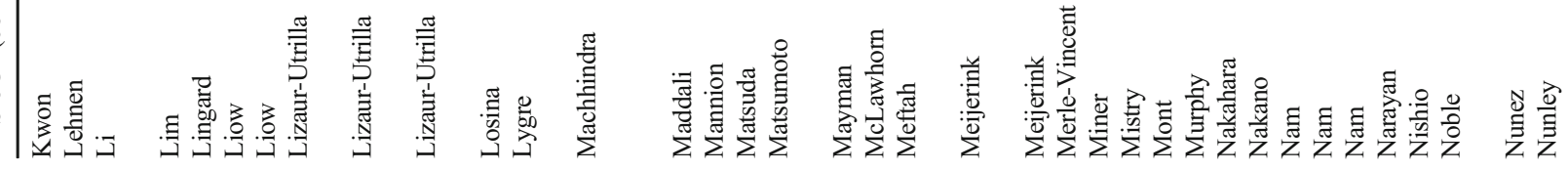




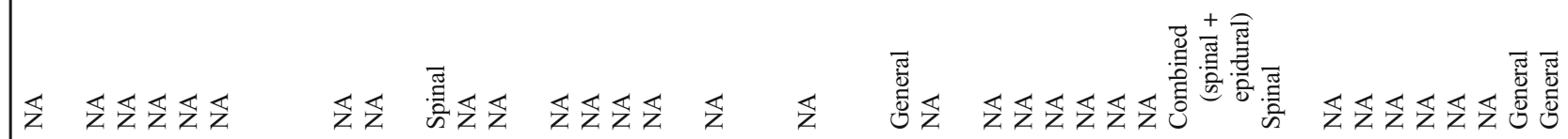
일

离

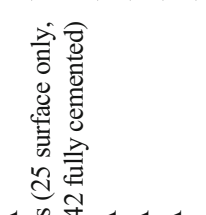

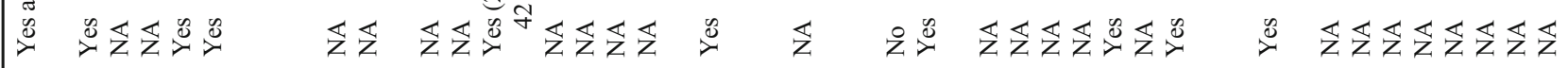
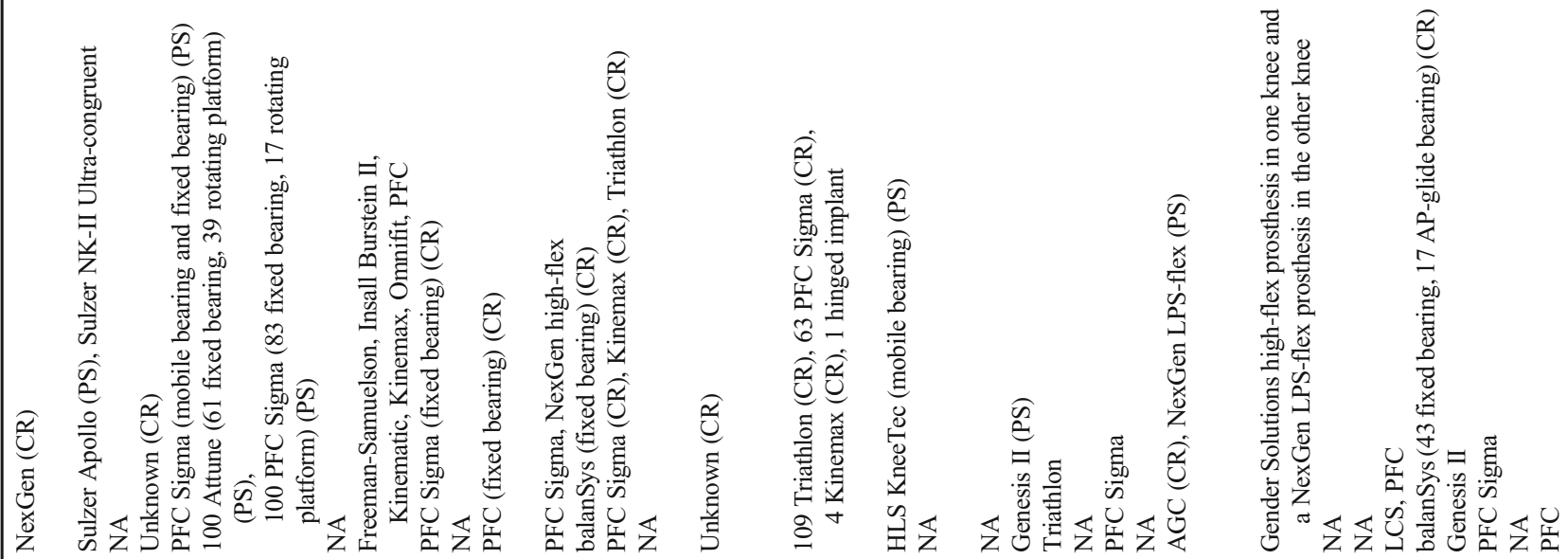

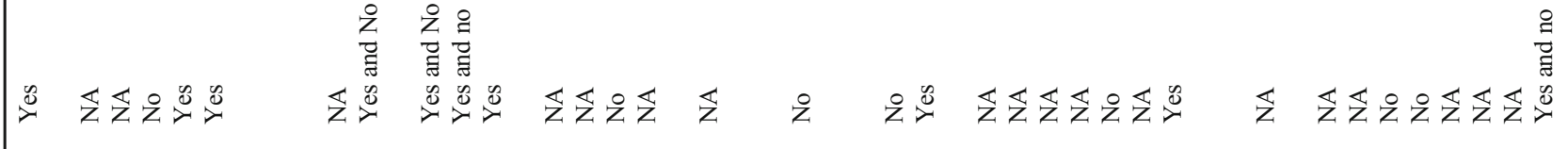

$\frac{0}{\overline{7}}$

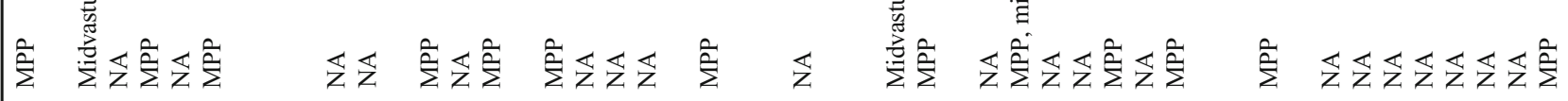

突

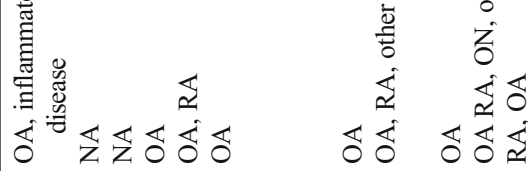

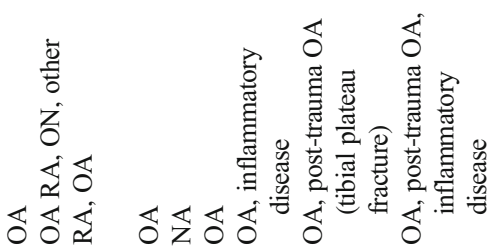

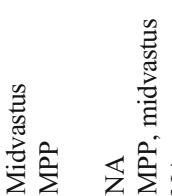<smiles>C[C@@H]1CC[C@H]1C1CC1</smiles>

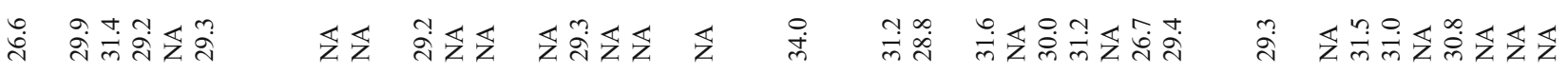

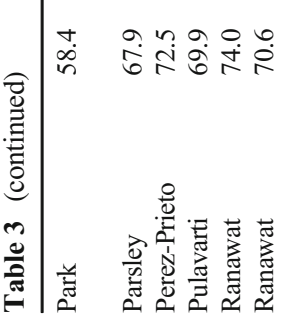

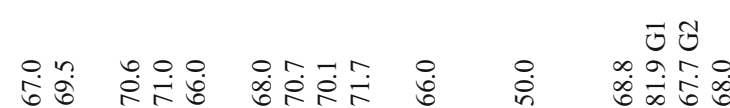

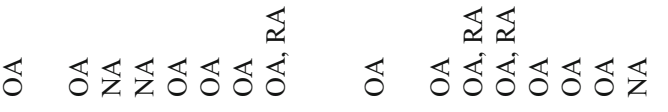


assessed using the Joanna Briggs Institute Critical Appraisal Checklist (JBICAC) for cohort studies, case-control studies, cross-sectional studies and case series [14]. For RCTs, a modified version of critical appraisal checklist by van Tulder et al. was used [15].

\section{Statistical methods}

Statistical analysis in this study focused on descriptive statistics. After assessing the quality of each study, the score was converted into a percentage from the full score (\%), which was then considered to be the 'strength' of that particular study. Microsoft Excel 2013 was used for our analysis in reporting the factors affecting patient satisfaction following a TKR, based on the strength of studies as per the type of evidence. The potential factors were then categorised into seven groups designed from the findings of the studies included. The strength of each factor was presented, regardless of whether it was a FACTOR ('it is a factor for patient satisfaction') or a Not-FACTOR ('it is a factor which does NOT relate to patient satisfaction' - in other words, 'researcher X found Factor Z was irrelevant to patient satisfaction').

Details are described in Electronic Supplementary Material 1 and Table 2.

\section{Results}

A total of 5635 articles were found following the initial search of the electronic databases and citation tracking, followed by removing 2424 duplicate articles. After review by title and abstract, 2977 articles were excluded and 234 potential articles remained for a full-text review. After application of the inclusion and exclusion criteria, a further 53 articles were discarded, leaving 181 relevant articles for the final inclusion, analysis and assessment. The study finally included 40 RCTs (22.1\%), 93 cohort studies (51.4\%), nine case-control studies (5.0\%), 37 cross-sectional studies $(20.4 \%)$ and 2 case series (1.1\%) (Electronic Supplementary Material 2). Flowchart for the review is shown in Fig. 1 and the details of all the 181 studies are shown in Table 3. A total of 22 authors were found to have written several papers. To ensure that duplicate numbers were not included in our analysis, we contacted all these authors and reminder emails were sent as well to ensure a reply. Only five authors replied back with no overlap in their studies, three authors said that there was an overlap and 14 did not reply back. Those who did not reply back were treated as if it was an overlap and, thus, not considered. Due to the lack of homogeneity between studies, a meta-analysis was deemed unsuitable for this study.

From all these studies, we found 98 factors, which could potentially affect patient satisfaction and these were then categorised into seven groups as follows: 
Table 4 Potential factors for patient satisfaction following primary total knee replacement (TKR) with their groups

\begin{tabular}{|c|c|c|}
\hline Factors & Sub-factors for satisfaction & Serial number of reporting studies \\
\hline \multicolumn{3}{|l|}{ 1. Patient demographics (47) } \\
\hline \multirow[t]{3}{*}{ Age (17) } & Young & $95,118,137,149(4)$ \\
\hline & Old & $9,112,125,172(4)$ \\
\hline & Not-FACTOR & $1,7,40,47,72,126,147,158,176(9)$ \\
\hline \multirow[t]{3}{*}{ Gender (10) } & Male & $9,14,133,149(4)$ \\
\hline & Female & $147(1)$ \\
\hline & Not-FACTOR & $7,40,72,126,176(5)$ \\
\hline \multirow[t]{2}{*}{ Body mass index (BMI), weight (12) } & Normal BMI & $10,23,62,98,125(5)$ \\
\hline & Not-FACTOR & $7,47,72,126,162,176,179(7)$ \\
\hline \multirow[t]{2}{*}{ Ethnicity (2) } & Caucasian $>$ African American & $71(1)$ \\
\hline & Not-FACTOR & $14(1)$ \\
\hline Income (2) & Annual income $>25,000$ USD & $14,133(2)$ \\
\hline \multirow{2}{*}{$\begin{array}{l}\text { Social background } \\
\text { (education, employment, insurance) (4) }\end{array}$} & High education & $62(1)$ \\
\hline & Not-FACTOR & $14,80,133(3)$ \\
\hline \multicolumn{3}{|l|}{ 2. Non-knee factors (30) } \\
\hline Back pain (3) & No low back pain & $34,42,153(3)$ \\
\hline Allergy (2) & No allergy & $121,132(2)$ \\
\hline Fibromyalgia (1) & No fibromyalgia & $15(1)$ \\
\hline Problems in other joints (2) & No problem in other joints & $117,153(2)$ \\
\hline General condition (1) & ASA 2 or worse & $9(1)$ \\
\hline Comorbidity (1) & No medical comorbidity & $7(1)$ \\
\hline Use of narcotics (1) & No use of narcotics & $51(1)$ \\
\hline Diabetes mellitus (1) & Not-FACTOR & $35(1)$ \\
\hline Generalised joint laxity (1) & Not-FACTOR & $102(1)$ \\
\hline \multirow[t]{2}{*}{$\begin{array}{l}\text { Mental health anxiety, } \\
\text { depression and personality traits (15) }\end{array}$} & No mental problem & $\begin{array}{l}3,5,7,16,17,23,33,47,49,54,55 \\
\quad 125,153(13)\end{array}$ \\
\hline & Not-FACTOR & $94,142(2)$ \\
\hline Pre-operative activity level (2) & Not-FACTOR & $128,171(2)$ \\
\hline \multicolumn{3}{|l|}{ 3. Knee factors (25) } \\
\hline Pre-operative stiff knee (1) & No stiff knee & $156(1)$ \\
\hline \multirow[t]{3}{*}{ Pre-operative knee pain (4) } & No pain at rest & $22,73(2)$ \\
\hline & Chronic pain & $149(1)$ \\
\hline & No movement-elicited pain & $73(1)$ \\
\hline History of past knee surgery (ACL reconstruction, HTO) (1) & Not-FACTOR & $106(1)$ \\
\hline Satisfaction on the first side (in bilateral TKR) (1) & Not-FACTOR & $154(1)$ \\
\hline \multirow[t]{4}{*}{ Diagnosis $(7)$} & $\mathrm{RA}>\mathrm{OA}$ & $24(1)$ \\
\hline & Not OA & $147(1)$ \\
\hline & $\mathrm{RA}>\mathrm{OA}>$ post-trauma $>\mathrm{AVN}$ & $149(1)$ \\
\hline & Not-FACTOR & $7,56,155,176(4)$ \\
\hline Degree of degeneration (4) & $\begin{array}{l}\text { Severe pre-operative radiographic } \\
\text { degenerative change }\end{array}$ & $71,79,125,151(4)$ \\
\hline Chondromalacia patellae (1) & Not-FACTOR & $181(1)$ \\
\hline Patellar congruence (1) & Not-FACTOR & $157(1)$ \\
\hline Intact ACL in CR-TKR (1) & No intact ACL & $70(1)$ \\
\hline Knee extensor strength (1) & Great knee extensor strength & $53(1)$ \\
\hline Intra-operative joint force (1) & $\begin{array}{l}\text { Greater intra-operative force in the medial } \\
\text { compartment }\end{array}$ & $74(1)$ \\
\hline Intra-operative kinematic pattern of the knee (1) & Medial pivot kinematic pattern & $136(1)$ \\
\hline Patient's perspective (1) & High flexion activities & $89(1)$ \\
\hline
\end{tabular}

4. Factors related to implants/prostheses (46) 
Table 4 (continued)

\begin{tabular}{|c|c|c|}
\hline Factors & Sub-factors for satisfaction & Serial number of reporting studies \\
\hline \multirow[t]{6}{*}{ Specific prosthesis (7) } & Triathlon > Kinemax & $60(1)$ \\
\hline & Triathlon $>$ Kinemax Plus & $46(1)$ \\
\hline & $\mathrm{PFC}>\mathrm{CKS}$ & $123(1)$ \\
\hline & Vega, Genesis II > E.motion & $75(1)$ \\
\hline & NexGen $>$ AGC & $114(1)$ \\
\hline & Not-FACTOR & $81,145(2)$ \\
\hline \multirow{2}{*}{$\begin{array}{l}\text { Cruciate-retaining/posterior-stabilised/ultra-congruent } \\
\text { design (8) }\end{array}$} & $\mathrm{PS}>\mathrm{CR}$ & $178(1)$ \\
\hline & Not-FACTOR & $14,18,44,78,115,141,165(7)$ \\
\hline \multirow[t]{3}{*}{ Design of the bearing (insert) (12) } & Mobile-bearing insert & $32,82,91,110(4)$ \\
\hline & Rotating mobile $>$ floating mobile & $83(1)$ \\
\hline & Not-FACTOR & $14,63,122,133,139,144,177(7)$ \\
\hline \multirow[t]{2}{*}{ Single radius prosthesis/multi-radius prosthesis (2) } & Single radius $>$ multi-radius & $43(1)$ \\
\hline & Not-FACTOR & $65(1)$ \\
\hline Use/type/number of stem (1) & Not-FACTOR & $12(1)$ \\
\hline Highly cross-linked polyethylene (1) & Not-FACTOR & $93(1)$ \\
\hline Material of femoral components (1) & Not-FACTOR & $67(1)$ \\
\hline Gender-specific design (6) & Not-FACTOR & $14,84,88,133,139,166(6)$ \\
\hline High-flexion design (7) & Not-FACTOR & $14,20,31,87,133,139,168(7)$ \\
\hline Customised prosthesis (1) & Non-customised (= off-the-shelf) prosthesis & $175(1)$ \\
\hline \multicolumn{3}{|l|}{ 5. Intra-operative technical factors (44) } \\
\hline \multirow[t]{2}{*}{ Approach, incision (4) } & Lateral subvastus approach & $66(1)$ \\
\hline & Not-FACTOR & $6,9,160(3)$ \\
\hline \multirow[t]{2}{*}{ Cement technique (4) } & $\begin{array}{l}\text { Surface-cemented }>\text { fully cemented (for tibial } \\
\text { component) }\end{array}$ & $150(1)$ \\
\hline & Not-FACTOR & $52,92,140(3)$ \\
\hline Kinematic alignment technique (1) & Not-FACTOR & $133(1)$ \\
\hline Gap balancing/measured resection technique (1) & Not-FACTOR & $41(1)$ \\
\hline \multirow{2}{*}{$\begin{array}{l}\text { Navigation/patient-specific instrument/custom cutting } \\
\text { guide/robotic surgery (13) }\end{array}$} & Using a navigation system & $104(1)$ \\
\hline & Not-FACTOR & $\begin{array}{l}19,39,61,96,97,99,108,109,131, \\
\quad 133,134,161(12)\end{array}$ \\
\hline \multirow[t]{2}{*}{ Patellar resurfacing (13) } & Patellar resurfacing & $120,148,149,174(4)$ \\
\hline & Not-FACTOR & $4,8,13,25,26,27,69,85,114(9)$ \\
\hline Lateral retinacular release $(1)$ & Not-FACTOR & $180(1)$ \\
\hline Minimally invasive surgery (MIS) (1) & Not-FACTOR & $64(1)$ \\
\hline Periarticular injection with corticosteroid (1) & Not-FACTOR & $103(1)$ \\
\hline \multirow[t]{2}{*}{ Patellar treatment (in cases without patellar resurfacing) (2) } & Patellar denervation & $143(1)$ \\
\hline & Patelloplasty & $163(1)$ \\
\hline Use of a tourniquet (1) & Not-FACTOR & $9(1)$ \\
\hline Removal of fat pad (1) & Not-FACTOR & $9(1)$ \\
\hline One-stage/two-stage bilateral TKR (1) & Not-FACTOR & $116(1)$ \\
\hline \multicolumn{3}{|l|}{ 6. Post-operative outcome factors (55) } \\
\hline Knee alignment (1) & Good post-operative alignment & $118(1)$ \\
\hline \multirow[t]{2}{*}{ Pain $(8)$} & No/less pain & $2,3,7,9,29,48,170(7)$ \\
\hline & No neuropathic pain & $146(1)$ \\
\hline \multirow[t]{2}{*}{ Range of motion (9) } & Improvement in ROM & $3,47,59,72,86,176(6)$ \\
\hline & Not-FACTOR & $45,126,135(3)$ \\
\hline Flexion contracture (2) & No flexion contracture & $32,57(2)$ \\
\hline Knee swelling (1) & No knee swelling & $47(1)$ \\
\hline Radiologic leg length discrepancy (2) & Not-FACTOR & $30,90(2)$ \\
\hline
\end{tabular}


Table 4 (continued)

\begin{tabular}{|c|c|c|}
\hline Factors & Sub-factors for satisfaction & Serial number of reporting studies \\
\hline Perception of leg length discrepancy (1) & No perception of leg length discrepancy & $30(1)$ \\
\hline \multirow[t]{4}{*}{ Malpositioning of femoral component (4) } & Accurate coronal alignment & $68(1)$ \\
\hline & $\begin{array}{l}\text { Medial malpositioned femoral component } \\
\text { (more than } 5 \mathrm{~mm})\end{array}$ & $169(1)$ \\
\hline & Accurate rotation & $77(1)$ \\
\hline & Not-FACTOR & $129(1)$ \\
\hline Malpositioning of tibial component (1) & Not-FACTOR & $77(1)$ \\
\hline Residual symptom (1) & No residual symptoms & $137(1)$ \\
\hline Physical function (7) & Good physical function & $7,11,36,48,117,130,137(7)$ \\
\hline Degree of expectation met (5) & Pre-operative expectations met & $21,22,40,137,164(5)$ \\
\hline Anterior-posterior knee stability (1) & Not-FACTOR & $152(1)$ \\
\hline Ligament balance (3) & Good ligament balance of the knee & $58,76,119(3)$ \\
\hline Medial joint laxity (1) & No medial joint laxity & $167(1)$ \\
\hline Lateral joint laxity (1) & Not-FACTOR & $167(1)$ \\
\hline Noise (2) & Not-FACTOR & $100,159(2)$ \\
\hline \multirow[t]{2}{*}{ Altered sensation (2) } & No numbness & $159(1)$ \\
\hline & Not-FACTOR & $127(1)$ \\
\hline \multirow[t]{2}{*}{ Complication (3) } & No complication & $22,125(2)$ \\
\hline & No deep prosthetic infection & $5(1)$ \\
\hline \multicolumn{3}{|l|}{ 7. Surgeon and healthcare factors (11) } \\
\hline Type of analgesia used (1) & Not-FACTOR & $173(1)$ \\
\hline Post-operative irrigation (1) & $\begin{array}{l}\text { Continuous irrigation by cold saline with } \\
\text { epinephrine }\end{array}$ & $105(1)$ \\
\hline \multirow[t]{2}{*}{ Post-operative rehabilitation (2) } & Patients' high motivation & $47(1)$ \\
\hline & Regular physical activity & $28(1)$ \\
\hline \multirow[t]{2}{*}{ Length of hospital stay (2) } & Short hospital stay & $5(1)$ \\
\hline & Not-FACTOR & $176(1)$ \\
\hline Waiting time before TKR (1) & Shorter than 6 months & $111(1)$ \\
\hline Country where TKR is conducted (1) & Not-FACTOR & $107(1)$ \\
\hline Surgeon's job title (consultant or not) (1) & Not-FACTOR & $9(1)$ \\
\hline $\begin{array}{l}\text { Surgeon's perspective towards the TKR } \\
\text { (surgeon's satisfaction) (1) }\end{array}$ & Not-FACTOR & $124(1)$ \\
\hline Hospital choice (1) & Patients having a hospital choice & $113(1)$ \\
\hline \multicolumn{3}{|l|}{ (Relating scores/scales) (17) } \\
\hline \multirow[t]{6}{*}{ Relation (+) } & WOMAC score & $86,126,138,164(4)$ \\
\hline & Oxford Knee Score & $37,38,68,153,156,176(6)$ \\
\hline & Knee Society Score & $72,176(2)$ \\
\hline & SF-12 score & $153,176(2)$ \\
\hline & SF-36 score & $164(1)$ \\
\hline & Control Preference Scale & $50(1)$ \\
\hline Relation (-) & New Knee Society Score & $101(1)$ \\
\hline
\end{tabular}

Reporting studies are described using serial numbers in Table 3. The number of each category is shown in parentheses Not-FACTOR 'it is a factor which does NOT relate to patient satisfaction'

1. Patient demographics

2. Non-knee factors

3. Knee factors

4. Factors relating to implants/prostheses

5. Intra-operative technical factors
6. Post-operative outcome factors

7. Surgeon and healthcare factors

All the 98 factors as well as scales/scores which were reported to relate to patient satisfaction are summarised in 


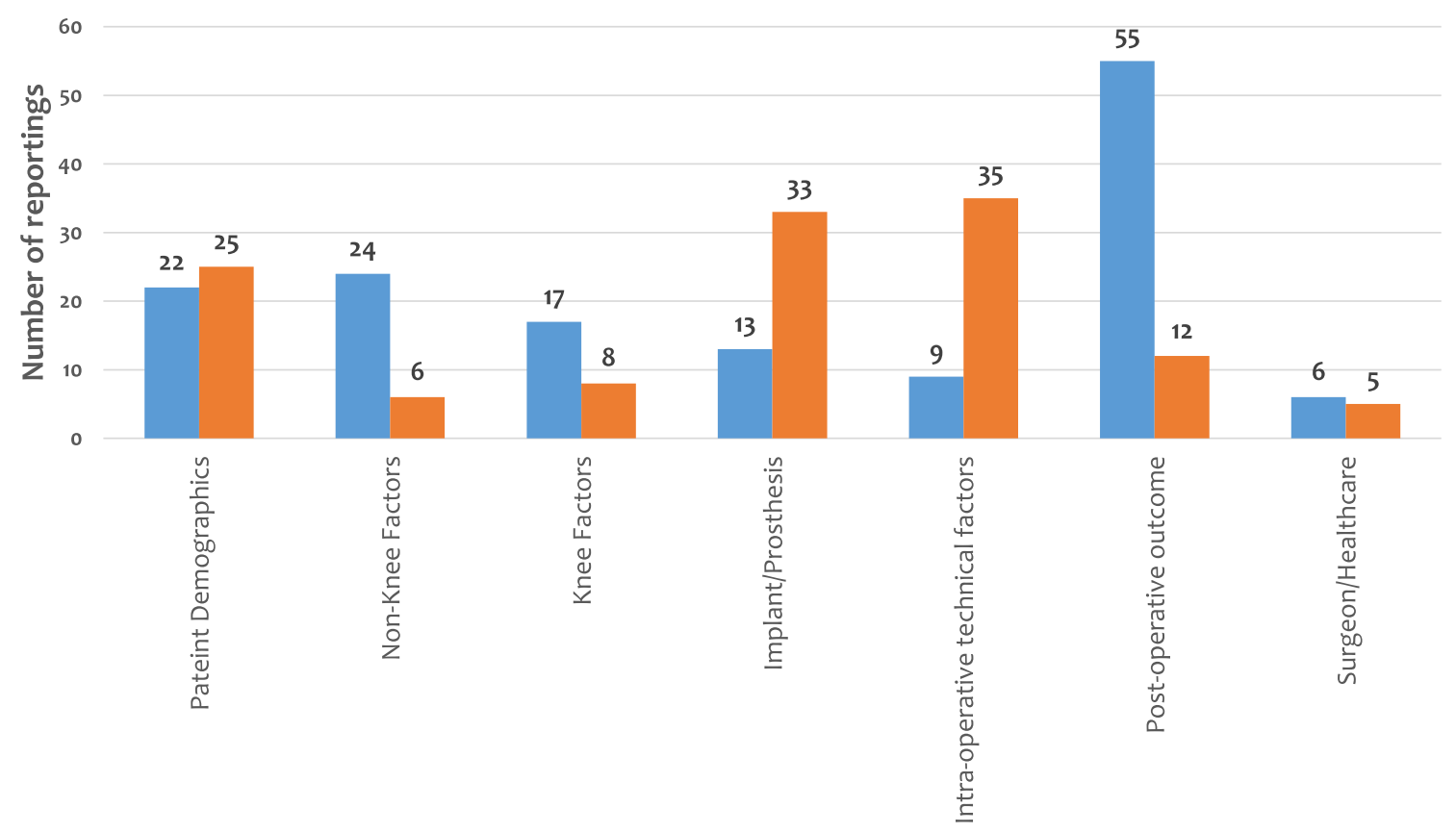

Fig. 2 Number of reportings in seven groups of factors for patient satisfaction following total knee replacement. Blue bar means FACTOR ('it is a factor for patients' satisfaction') and orange bar means Not-FACTOR ('it is a factor which does NOT relate to patients' satisfaction')

Table 4. Details of the results in each group are described in Electronic Supplementary Material 3. The number of reportings for each group is presented in Fig. 2, and the methods used to measure satisfaction are shown in Table 5.

The quality of all the 181 studies was assessed and the results are shown in Tables 6, 7, 8, 9 and 10. The strength of each factor was described using the sum of percentage in each type of study (RCT, cohort study, case-control study, crosssectional study and case series) (Fig. 3). RCTs were considered to be the strongest (deep colour in Fig. 3) and this was followed by cohort study, case-control study and crosssectional study, respectively. Case series was considered to be the weakest (light colour in Fig. 3).

When the results of the quality assessment were taken into consideration, a negative history of mental health problems, use of a mobile-bearing insert, patellar resurfacing, severe preoperative radiological degenerative change, negative history of low back pain, no/less post-operative pain, good postoperative physical function and pre-operative expectations being met were considered to be important factors. Significant factors affecting patient satisfaction are summarised in Tables 11, 12 and 13.

\section{Discussion}

The dissatisfaction rate following a TKR remains around $20 \%$ and is a constant source of frustration for the patient and the surgeon $[11,12]$. Our study has systematically reviewed all the articles looking at satisfaction following a TKR to determine the factors, which could be responsible for this issue. Several factors were deemed to be important in affecting patient satisfaction based on the number of studies in which they were reported as well as the results of the quality assessment of the study (Tables 11, 12 and 13).

\section{Negative history of mental health problems}

A negative history of mental health problems was the most frequently reported factor affecting patient satisfaction (Table 11) and also scored the highest sum of percentage of FACTOR based on the quality assessment for RCT + cohort study ( \pm case-control study \pm cross-sectional study \pm case series study) (Table 12). In addition, it was ranked first in terms of the highest sum of percentage of FACTOR and Not-FACTOR based on the quality assessment for all types of the studies (Table 13). Depressive symptoms and anxiety were reported to be predictive of long-term pain and functional impairment as measured by the Knee Society Score in 83 patients at 5 years [16]. In addition, it was reported that preoperative anxiety/depression is an independent risk for severe post-operative pain and may explain as to why there is a subset of patients with unexplained pain after surgery [17]. Moreover, Macleod et al. report that patients with mental disability suffered a greater level of comorbidity and were socially deprived, which is also related to poorer physical health which then has an impact on satisfaction [18]. Finally, another study reported that patients with poor mental health, which can impair coping mechanisms for pain, might present with 
Table 5 Measuring methods for patients' satisfaction

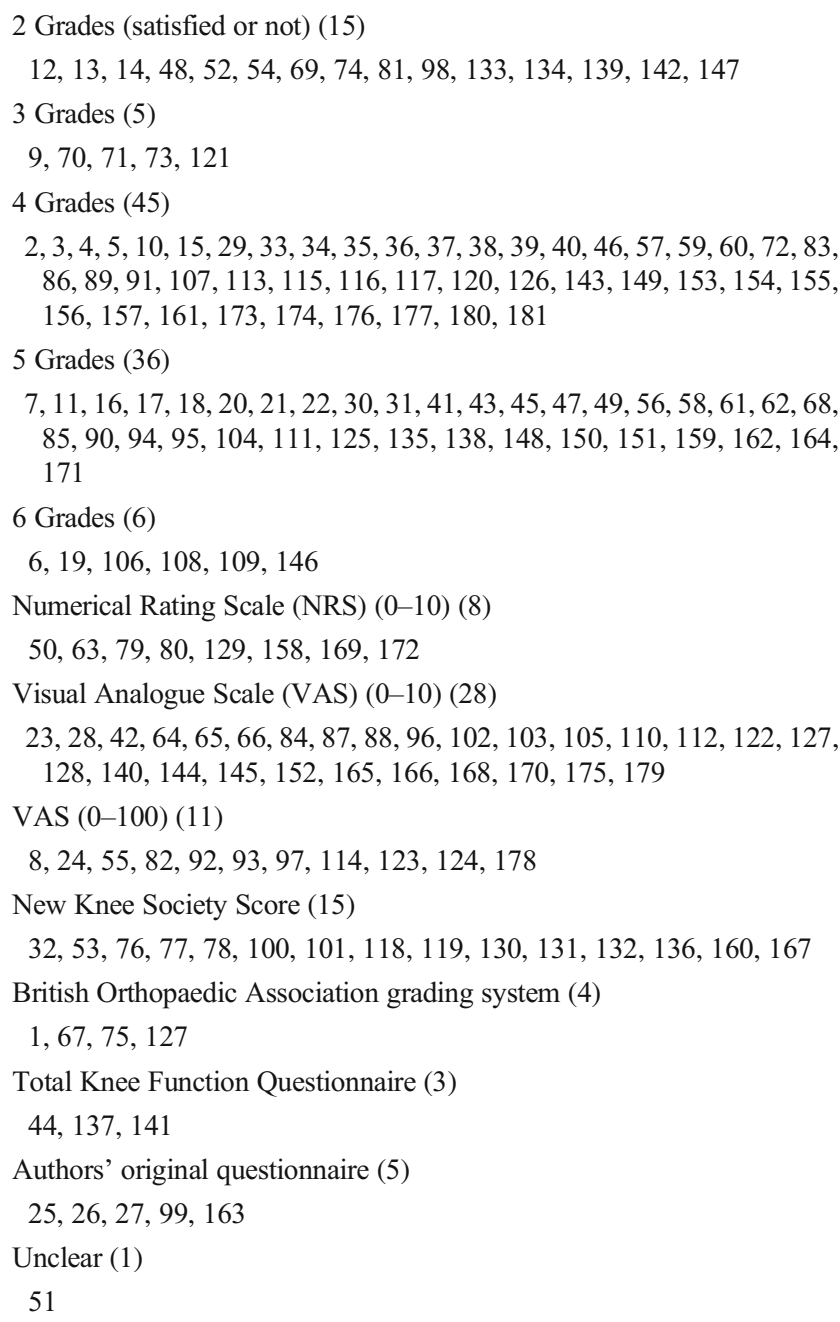

Studies are described using serial numbers in Table 3. The number of studies in each group is shown in parentheses

less severe disease, and this could also influence their satisfaction [19].

\section{Use of a mobile-bearing insert}

The use of a mobile-bearing insert had the highest sum of percentage of FACTOR based on the quality assessment for RCTs. Also, it had the second highest sum of percentage of FACTOR based on the quality assessment for RCT + cohort study ( \pm case-control study) (Table 12 ). The rationale behind the design of a mobile-bearing insert is to solve the kinematic conflict between low-stress articulation and free axial femoral-tibial rotation by allowing rotation of a highly conforming polyethylene insert [20]. Theoretically, the design of the mobile-bearing insert could lead to better ROM especially during flexion [21]. A greater loss of flexion was reported after 12 months in patients with a TKR with a fixed-bearing prosthesis in comparison with a mobile-bearing prosthesis [22]. It is quite intuitive to comprehend that a good post-operative ROM relates to patient satisfaction, and our results support this (improvement in ROM was the 4th most frequently reported factor for patient satisfaction). Kim et al. suspect the low constraint of mobile-bearing insert may restore normal kinematics of the knee and it contributes to favourable clinical outcomes compared with a fixed-bearing insert [23]. Price et al. in a prospective multicentre trial of 39 simultaneous bilateral procedures also found that patients with a mobile-bearing insert had significantly better clinical results than patients with a fixed-bearing insert [21].

\section{Patellar resurfacing}

Patellar resurfacing has the second highest sum of percentage of FACTOR based on the quality assessment for RCTs (Table 12). Four studies showed patients with patella resurfacing were more satisfied than those without it [11, 24-26]. Amongst them, one study focused on only knees with no exposed bone on the undersurface of the patella to determine the potential advantages of leaving the patella non-resurfaced [25]. Dissatisfaction in patella non-resurfaced patients may be due to the higher rate of post-operative anterior knee pain, and patients whose patella was not resurfaced at the index TKR tended to have a higher revision rate as well [25-28]. However, it should be noted that this issue may be strongly related to the design of the implant. There have also been abundant literature that showed that the patellofemoral design in TKR is critical and can vary the forces on the patellofemoral joint as well as patellofemoral tracking [29-31]. Two of the 4 studies relate to a specific prosthesis (PFC) which is notoriously patella unfriendly $[25,26]$, so this relationship may therefore not necessarily hold true for the newer implants with patella-friendly designs.

\section{Severe pre-operative radiological degenerative change}

Severe pre-operative radiological degenerative change has the fifth highest sum of percentage of FACTOR and Not-FACTOR based on the quality assessment for all types of studies (Table 13). Although the classic indication for replacing a patient's knee is end-stage arthritis (Kellgren-Lawrence grade IV [32]), there are a number of patients who have a TKR much before grade IV radiological changes have set in and it is dependent on the symptoms of the patient. The individual indication is complex and involves multiple factors [33]. 
Table 6 Results of quality assessment of 181 studies — cohort studies: 93 studies. The Joanna Briggs Institute Critical Appraisal Checklist is used

Scoring: Yes $=2$ / Unclear $=1 /$ No $=0 / \mathrm{NA}=$ not applicable

Q1: Were the two groups similar and recruited from the same population?

Q2: Were the exposures measured similarly to assign people to both exposed and unexposed groups?

Q3: Was the exposure measured in a valid and reliable way?

Q4: Were confounding factors identified?

Q5: Were strategies to deal with confounding factors stated?

Q6: Were the groups/participants free of the outcome at the start of the study (or at the moment of exposure)?

Q7: Were the outcomes measured in a valid and reliable way?

Q8: Was the follow-up time reported and sufficient to be long enough for outcomes to occur?

Q9: Was follow-up complete, and if not, were the reasons to lose to follow-up described and explored?

Q10: Were strategies to address incomplete follow-up utilised?

Q11: Was appropriate statistical analysis used?

\begin{tabular}{|c|c|c|c|c|c|c|c|c|c|c|c|c|c|}
\hline Study (serial no.) & Q1 & Q2 & Q3 & Q4 & Q5 & Q6 & Q7 & Q8 & Q9 & Q10 & Q11 & Total ( /22) & $\%$ \\
\hline 1 & 2 & 2 & 2 & 1 & 1 & 2 & 2 & 2 & 2 & 1 & 2 & 19 & 86.4 \\
\hline 5 & 1 & 1 & 2 & 0 & 0 & 0 & 2 & 2 & 2 & 2 & 2 & 14 & 63.6 \\
\hline 6 & 2 & 2 & 1 & 1 & 0 & 2 & 2 & 2 & 2 & 2 & 2 & 18 & 81.8 \\
\hline 7 & 0 & 2 & 2 & 0 & 0 & 2 & 2 & 2 & 2 & 2 & 2 & 16 & 72.7 \\
\hline 10 & 2 & 2 & 2 & 2 & 2 & 1 & 2 & 2 & 2 & 2 & 2 & 21 & 95.5 \\
\hline 11 & 0 & 1 & 2 & 0 & 0 & 2 & 2 & 2 & 2 & 2 & 1 & 14 & 63.6 \\
\hline 12 & 2 & 2 & 2 & 0 & 2 & 2 & 2 & 2 & 2 & 1 & 2 & 19 & 86.4 \\
\hline 14 & 0 & 1 & 1 & 1 & 1 & 2 & 2 & 2 & 2 & 1 & 2 & 15 & 68.2 \\
\hline 16 & 0 & 0 & 2 & 0 & 0 & 1 & 2 & 2 & 2 & 2 & 1 & 12 & 54.5 \\
\hline 17 & 0 & 0 & 2 & 0 & 0 & 1 & 2 & 2 & 2 & 2 & 2 & 13 & 59.1 \\
\hline 18 & 2 & 2 & 2 & 0 & 0 & 2 & 2 & 2 & 2 & 1 & 1 & 16 & 72.7 \\
\hline 23 & 1 & 1 & 2 & 2 & 2 & 2 & 2 & 2 & 2 & 1 & 2 & 19 & 86.4 \\
\hline 28 & 0 & 2 & 2 & 2 & 2 & 2 & 2 & 2 & 2 & 1 & 2 & 19 & 86.4 \\
\hline 30 & 0 & 1 & 1 & 0 & 0 & 2 & 2 & 2 & 2 & 1 & 2 & 13 & 59.1 \\
\hline 32 & 2 & 2 & 2 & 1 & 1 & 2 & 2 & 2 & 2 & 1 & 2 & 19 & 86.4 \\
\hline 33 & 0 & 2 & 2 & 2 & 2 & 1 & 2 & 2 & 1 & 0 & 2 & 16 & 72.7 \\
\hline 34 & 2 & 2 & 2 & 2 & 2 & 2 & 2 & 2 & 2 & 0 & 2 & 20 & 90.9 \\
\hline 35 & 0 & 2 & 2 & 2 & 2 & 1 & 2 & 2 & 1 & 0 & 2 & 16 & 72.7 \\
\hline 36 & 2 & 2 & 2 & 1 & 2 & 1 & 2 & 2 & 1 & 1 & 2 & 18 & 81.8 \\
\hline 37 & 0 & 2 & 2 & 0 & 0 & 1 & 2 & 2 & 2 & 0 & 2 & 13 & 59.1 \\
\hline 39 & 2 & 2 & 2 & 0 & 0 & 1 & 2 & 2 & 2 & 0 & 2 & 15 & 68.2 \\
\hline 40 & 0 & 2 & 2 & 0 & 0 & 2 & 2 & 2 & 2 & 0 & 2 & 14 & 63.6 \\
\hline 41 & 2 & 2 & 2 & 2 & 2 & 2 & 2 & 2 & 2 & 0 & 2 & 20 & 90.9 \\
\hline 42 & 2 & 2 & 2 & 0 & 0 & 2 & 2 & 2 & 2 & 2 & 1 & 17 & 77.3 \\
\hline 44 & 2 & 2 & 2 & 2 & 1 & 2 & 2 & 2 & 2 & 0 & 2 & 19 & 86.4 \\
\hline 46 & 2 & 2 & 2 & 0 & 0 & 2 & 2 & 2 & 2 & 0 & 2 & 16 & 72.7 \\
\hline 49 & 0 & 2 & 2 & 1 & 1 & 2 & 2 & 2 & 2 & 1 & 1 & 16 & 72.7 \\
\hline 50 & 0 & 2 & 2 & 0 & 0 & 1 & 2 & 2 & 1 & 1 & 2 & 13 & 59.1 \\
\hline 51 & 2 & 2 & 2 & 0 & 0 & 2 & 2 & 2 & 1 & 0 & 2 & 15 & 68.2 \\
\hline 53 & 0 & 2 & 2 & 0 & 0 & 2 & 2 & 2 & 2 & 0 & 2 & 14 & 63.6 \\
\hline 54 & 0 & 2 & 2 & 0 & 0 & 2 & 2 & 2 & 1 & 0 & 2 & 13 & 59.1 \\
\hline 56 & 2 & 2 & 2 & 2 & 2 & 2 & 2 & 2 & 2 & 0 & 2 & 20 & 90.9 \\
\hline 57 & 2 & 2 & 2 & 0 & 0 & 2 & 2 & 2 & 2 & 0 & 2 & 16 & 72.7 \\
\hline 58 & 2 & 2 & 2 & 0 & 0 & 2 & 2 & 2 & 1 & 0 & 2 & 15 & 68.2 \\
\hline 59 & 0 & 2 & 2 & 0 & 0 & 2 & 2 & 2 & 2 & 0 & 2 & 14 & 63.6 \\
\hline 65 & 2 & 1 & 2 & 0 & 0 & 2 & 2 & 2 & 2 & 0 & 2 & 15 & 68.2 \\
\hline 66 & 2 & 2 & 2 & 2 & 2 & 2 & 2 & 2 & 2 & 1 & 2 & 21 & 95.5 \\
\hline 68 & 0 & 2 & 2 & 0 & 0 & 2 & 2 & 2 & 1 & 0 & 2 & 13 & 59.1 \\
\hline 70 & 0 & 2 & 2 & 1 & 0 & 2 & 2 & 2 & 1 & 0 & 2 & 14 & 63.6 \\
\hline 73 & 0 & 2 & 2 & 0 & 0 & 2 & 2 & 2 & 2 & 0 & 2 & 14 & 63.6 \\
\hline
\end{tabular}


Table 6 (continued)

\begin{tabular}{|c|c|c|c|c|c|c|c|c|c|c|c|c|c|}
\hline 74 & 2 & 2 & 2 & 1 & 2 & 2 & 2 & 2 & 2 & 0 & 1 & 18 & 81.8 \\
\hline 75 & 2 & 2 & 2 & 2 & 2 & 2 & 2 & 2 & 2 & 0 & 2 & 20 & 90.9 \\
\hline 79 & 2 & 2 & 2 & 2 & 2 & 2 & 2 & 2 & 2 & 1 & 2 & 21 & 95.5 \\
\hline 80 & 2 & 2 & 2 & 2 & 2 & 2 & 2 & 2 & 2 & 1 & 2 & 21 & 95.5 \\
\hline 81 & 2 & 2 & 2 & 0 & 0 & 2 & 2 & 1 & 0 & 0 & 1 & 12 & 54.5 \\
\hline 83 & 2 & 2 & 2 & 2 & 2 & 2 & 2 & 2 & 2 & 0 & 2 & 20 & 90.9 \\
\hline 85 & 2 & 2 & 2 & 1 & 1 & 2 & 2 & 2 & 2 & 0 & 2 & 18 & 81.8 \\
\hline 90 & 2 & 2 & 2 & 0 & 0 & 2 & 2 & 2 & 2 & 0 & 2 & 16 & 72.7 \\
\hline 94 & 2 & 2 & 2 & 0 & 0 & 2 & 2 & 2 & 2 & 0 & 2 & 16 & 72.7 \\
\hline 95 & 2 & 2 & 2 & 0 & 0 & 2 & 2 & 2 & 2 & 0 & 2 & 16 & 72.7 \\
\hline 99 & 2 & 2 & 2 & 0 & 0 & 2 & 2 & 2 & 2 & 0 & 2 & 16 & 72.7 \\
\hline 101 & 0 & 2 & 2 & 0 & 0 & 2 & 2 & 2 & 1 & 0 & 2 & 13 & 59.1 \\
\hline 104 & 2 & 2 & 2 & 2 & 1 & 2 & 2 & 2 & 2 & 2 & 2 & 21 & 95.5 \\
\hline 105 & 2 & 2 & 2 & 0 & 0 & 2 & 2 & 2 & 2 & 0 & 2 & 16 & 72.7 \\
\hline 107 & 0 & 2 & 1 & 1 & 2 & 2 & 2 & 2 & 2 & 0 & 2 & 16 & 72.7 \\
\hline 111 & 2 & 2 & 2 & 0 & 1 & 2 & 2 & 2 & 2 & 1 & 2 & 18 & 81.8 \\
\hline 112 & 1 & 2 & 2 & 0 & 0 & 2 & 2 & 2 & 2 & 0 & 2 & 15 & 68.2 \\
\hline 115 & 2 & 2 & 2 & 2 & 2 & 2 & 2 & 2 & 1 & 0 & 2 & 19 & 86.4 \\
\hline 116 & 2 & 2 & 2 & 0 & 0 & 2 & 2 & 2 & 1 & 0 & 2 & 15 & 68.2 \\
\hline 119 & 0 & 2 & 2 & 1 & 1 & 2 & 2 & 2 & 2 & 0 & 2 & 16 & 72.7 \\
\hline 121 & 0 & 2 & 2 & 2 & 2 & 2 & 2 & 2 & 1 & 0 & 2 & 17 & 77.3 \\
\hline 122 & 2 & 2 & 2 & 0 & 0 & 2 & 2 & 2 & 1 & 0 & 2 & 15 & 68.2 \\
\hline 124 & 0 & 1 & 2 & 0 & 0 & 2 & 2 & 2 & 1 & 0 & 2 & 12 & 54.5 \\
\hline 125 & 0 & 2 & 1 & 0 & 0 & 2 & 2 & 2 & 2 & 0 & 2 & 13 & 59.1 \\
\hline 126 & 1 & 1 & 2 & 1 & 2 & 2 & 2 & 2 & 1 & 0 & 2 & 16 & 72.7 \\
\hline 127 & 1 & 2 & 2 & 0 & 0 & 2 & 2 & 1 & 1 & 0 & 1 & 12 & 54.5 \\
\hline 128 & 2 & 2 & 2 & 0 & 0 & 2 & 2 & 2 & 1 & 0 & 1 & 14 & 63.6 \\
\hline 131 & 2 & 2 & 2 & 0 & 0 & 2 & 2 & 2 & 2 & 0 & 1 & 15 & 68.2 \\
\hline 132 & 0 & 2 & 2 & 2 & 2 & 2 & 2 & 2 & 2 & 1 & 1 & 18 & 81.8 \\
\hline 134 & 1 & 2 & 2 & 0 & 0 & 2 & 2 & 2 & 2 & 0 & 1 & 14 & 63.6 \\
\hline 135 & 0 & 2 & 2 & 0 & 0 & 2 & 2 & 2 & 1 & 0 & 1 & 12 & 54.5 \\
\hline 138 & 1 & 2 & 2 & 2 & 1 & 2 & 2 & 2 & 2 & 0 & 2 & 18 & 81.8 \\
\hline 139 & 2 & 2 & 2 & 2 & 2 & 2 & 2 & 2 & 2 & 1 & 2 & 21 & 95.5 \\
\hline 141 & 2 & 2 & 2 & 0 & 0 & 2 & 2 & 2 & 1 & 0 & 2 & 15 & 68.2 \\
\hline 142 & 2 & 2 & 2 & 0 & 0 & 2 & 2 & 2 & 2 & 0 & 2 & 16 & 72.7 \\
\hline 144 & 2 & 2 & 2 & 1 & 0 & 2 & 2 & 2 & 2 & 0 & 2 & 17 & 77.3 \\
\hline 145 & 2 & 2 & 2 & 2 & 1 & 2 & 2 & 2 & 2 & 2 & 2 & 21 & 95.5 \\
\hline 150 & 2 & 2 & 2 & 0 & 0 & 2 & 2 & 2 & 2 & 0 & 2 & 16 & 72.7 \\
\hline 151 & 0 & 2 & 2 & 0 & 0 & 2 & 2 & 2 & 1 & 0 & 2 & 13 & 59.1 \\
\hline 152 & 0 & 2 & 2 & 0 & 0 & 2 & 2 & 2 & 2 & 0 & 2 & 14 & 63.6 \\
\hline 153 & 0 & 2 & 2 & 1 & 1 & 2 & 2 & 2 & 2 & 0 & 2 & 16 & 72.7 \\
\hline 154 & 0 & 2 & 2 & 0 & 0 & 2 & 2 & 2 & 2 & 1 & 2 & 15 & 68.2 \\
\hline 155 & 1 & 2 & 2 & 1 & 1 & 2 & 2 & 2 & 2 & 0 & 2 & 17 & 77.3 \\
\hline 156 & 0 & 2 & 2 & 1 & 1 & 2 & 2 & 2 & 2 & 0 & 2 & 16 & 72.7 \\
\hline 157 & 0 & 2 & 2 & 0 & 0 & 2 & 2 & 2 & 1 & 0 & 2 & 13 & 59.1 \\
\hline 158 & 2 & 2 & 2 & 0 & 0 & 2 & 2 & 2 & 2 & 0 & 2 & 16 & 72.7 \\
\hline 160 & 2 & 2 & 2 & 0 & 0 & 2 & 2 & 2 & 1 & 0 & 2 & 15 & 68.2 \\
\hline 161 & 2 & 2 & 2 & 0 & 1 & 2 & 2 & 2 & 2 & 0 & 2 & 17 & 77.3 \\
\hline 163 & 2 & 2 & 2 & 0 & 0 & 2 & 2 & 2 & 2 & 0 & 2 & 16 & 72.7 \\
\hline 164 & 0 & 2 & 2 & 0 & 0 & 2 & 2 & 2 & 1 & 0 & 2 & 13 & 59.1 \\
\hline 170 & 0 & 2 & 2 & 1 & 1 & 2 & 2 & 2 & 1 & 0 & 2 & 15 & 68.2 \\
\hline 172 & 2 & 2 & 2 & 1 & 1 & 2 & 2 & 2 & 2 & 0 & 2 & 18 & 81.8 \\
\hline 175 & 2 & 2 & 2 & 0 & 0 & 2 & 2 & 2 & 2 & 0 & 1 & 15 & 68.2 \\
\hline
\end{tabular}

Studies are described using serial numbers in Table 3

Patients with mild pre-operative OA were reported to have a worse prognosis in improvement in physical functioning [34, 35], and therefore, it is difficult to meet their expectations post-operatively [35]. These effects are more noticeable in patients undergoing a TKR as compared with those who have had a THR [34]. The knee is a complex joint and the biomechanics of this joint are much more difficult to replicate with a prosthetic knee as compared with a prosthetic hip which may partly explain a smaller increase in physical functioning and a poor rate of satisfaction in patients with mild OA having a TKR [36]. 
Table 7 Results of quality assessment of 181 studies — case-control studies: 9 studies. The Joanna Briggs Institute Critical Appraisal Checklist is used

Scoring: Yes $=2$ / Unclear $=1 / \mathrm{No}=0 / \mathrm{NA}=$ not applicableQ1: Were the groups comparable other than the presence of disease in cases or the absence of disease in controls?

Q2: Were cases and controls matched appropriately?

Q3: Were the same criteria used for identification of cases and controls?

Q4: Was exposure measured in a standard, valid and reliable way?

Q5: Was exposure measured in the same way for cases and controls?

Q6: Were confounding factors identified?

Q7: Were strategies to deal with confounding factors stated?

Q8: Were outcomes assessed in a standard, valid and reliable way for cases and controls?

Q9: Was the exposure period of interest long enough to be meaningful?

Q10: Was appropriate statistical analysis used?

\begin{tabular}{lllllllllllll} 
Study (serial no.) & Q1 & 1. Q2 & 2. Q3 & 3. Q4 & 4. Q5 & 5. Q6 & 6 . Q7 & 7. Q8 & 8. Q9 & 9. Q10 & Total (/20) & $\%$ \\
15 & 2 & 1 & 2 & 2 & 2 & 1 & 1 & 2 & 2 & 2 & 17 & 85.0 \\
20 & 1 & 1 & 1 & 2 & 2 & 1 & 1 & 2 & 0 & 2 & 13 & 65.0 \\
69 & 1 & 1 & 1 & 2 & 2 & 2 & 1 & 2 & 2 & 2 & 16 & 80.0 \\
93 & 2 & 2 & 2 & 2 & 2 & 0 & 0 & 2 & 2 & 2 & 16 & 80.0 \\
98 & 2 & 2 & 2 & 2 & 2 & 0 & 0 & 2 & 2 & 2 & 16 & 80.0 \\
102 & 1 & 1 & 1 & 2 & 2 & 0 & 0 & 2 & 2 & 2 & 13 & 65.0 \\
108 & 2 & 1 & 1 & 2 & 2 & 1 & 1 & 2 & 1 & 2 & 15 & 75.0 \\
114 & 2 & 2 & 2 & 2 & 2 & 2 & 2 & 2 & 2 & 2 & 20 & 100.0 \\
179 & 2 & 2 & 2 & 2 & 2 & 2 & 2 & 2 & 2 & 2 & 20 & 100.0 \\
\hline
\end{tabular}

Studies are described using Serial numbers in Table 3

\section{No low back pain}

No low back pain has the sixth highest sum of percentage of FACTOR and Not-FACTOR based on the quality assessment for all types of the studies (Table 13). The prevalence of chronic low back pain in the UK has been reported to range from 6 to $11 \%$ [29], and this is increased to $55 \%$ in patients with $\mathrm{OA}$ of the knee [30]. Furthermore, low back pain has been demonstrated to be three to four times more likely to be present in patients with a history of depression [37]. Also, patients with chronic low back pain have a higher rate of musculoskeletal and neuropathic pain conditions, depression, anxiety and sleep disorders [31]. In addition, patients with low back pain reported to have more symptoms from their osteoarthritic knee which may suggest a lower threshold for pain in this cohort leading to dissatisfaction [30].

\section{Normal BMI}

Normal BMI was the fifth most frequently reported factor for patient satisfaction (Table 11). BMI greater than 30 $\mathrm{kg} / \mathrm{m}^{2}$ was reported to be associated with a higher rate of revision and poorer functional outcomes as well which again contributes to dissatisfaction [38]. In addition, morbidly obese patients are likely to suffer from wound problems, ligament injuries and infections peri-operatively which lead to dissatisfaction [22]. Another study showed that despite lower pre- and post-operative WOMAC and SF-36 scores, obese patients experienced similar improvements compared with non-obese patients, although levels of satisfaction in the obese group were lower than those in the non-obese group [39]. The authors stated that one explanation for this might be that satisfaction was more closely associated with the absolute post-operative functional level rather than the magnitude of any improvement, as the rate of satisfaction mirrored absolute values of post-operative WOMAC and SF-36 scores.

\section{Other factors}

Other than factors discussed in the previous section, no/less post-operative pain, good post-operative physical function, improvement in ROM and pre-operative expectations being met were considered to be important for patient satisfaction based on the number of reportings and the results of quality assessment (Tables 11,12 and 13). TKR is a painful procedure and it does take at least six to 12 months to get the maximum benefit from this procedure [40], and therefore, setting realistic expectations with the patient in the pre-operative clinic is essential to avoid dissatisfaction.

\section{Limitations and strengths of the study}

Our study has several limitations. Firstly, the method of measuring satisfaction is different in each study, and therefore, a 
Table 8 Results of quality assessment of 181 studies — cross-sectional studies: 37 studies. The Joanna Briggs Institute Critical Appraisal Checklist is used

Scoring: Yes $=2 /$ Unclear $=1 /$ No $=0 / \mathrm{NA}=$ not applicable

Q1: Were the criteria for inclusion in the sample clearly defined?

Q2: Were the study subjects and the setting described in detail?

Q3: Was the exposure measured in a valid and reliable way?

Q4: Were objective, standard criteria used for measurement of the condition?

Q5: Were confounding factors identified?

Q6: Were strategies to deal with confounding factors stated?

Q7: Were the outcomes measured in a valid and reliable way?

Q8: Was appropriate statistical analysis used?

\begin{tabular}{|c|c|c|c|c|c|c|c|c|c|c|}
\hline Study (serial no.) & Q1 & Q2 & Q3 & Q4 & Q5 & Q6 & Q7 & Q8 & Total (/16) & $\%$ \\
\hline 2 & 2 & 2 & 2 & 2 & 1 & 1 & 2 & 2 & 14 & 87.5 \\
\hline 3 & 2 & 2 & 1 & 2 & 0 & 0 & 2 & 2 & 11 & 68.8 \\
\hline 9 & 2 & 2 & 2 & 2 & 1 & 1 & 2 & 2 & 14 & 87.5 \\
\hline 21 & 2 & 2 & 2 & 2 & 0 & 0 & 2 & 2 & 12 & 75.0 \\
\hline 22 & 2 & 2 & 2 & 2 & 0 & 0 & 2 & 2 & 12 & 75.0 \\
\hline 24 & 2 & 2 & 2 & 2 & 0 & 0 & 2 & 2 & 12 & 75.0 \\
\hline 29 & 1 & 2 & 2 & 2 & 2 & 2 & 2 & 2 & 15 & 93.8 \\
\hline 38 & 2 & 2 & 2 & 2 & 2 & 2 & 2 & 2 & 16 & 100.0 \\
\hline 45 & 1 & 2 & 2 & 2 & 1 & 1 & 2 & 2 & 13 & 81.3 \\
\hline 47 & 1 & 2 & 2 & 2 & 0 & 0 & 2 & 2 & 11 & 68.8 \\
\hline 48 & 2 & 2 & 2 & 2 & 0 & 0 & 2 & 2 & 12 & 75.0 \\
\hline 55 & 2 & 2 & 2 & 2 & 0 & 0 & 2 & 2 & 12 & 75.0 \\
\hline 62 & 2 & 2 & 2 & 2 & 1 & 2 & 2 & 2 & 15 & 93.8 \\
\hline 63 & 1 & 2 & 2 & 1 & 1 & 1 & 2 & 2 & 12 & 75.0 \\
\hline 71 & 1 & 2 & 2 & 2 & 1 & 2 & 2 & 2 & 14 & 87.5 \\
\hline 72 & 1 & 2 & 2 & 2 & 0 & 0 & 2 & 2 & 11 & 68.8 \\
\hline 77 & 1 & 1 & 2 & 2 & 0 & 0 & 2 & 2 & 10 & 62.5 \\
\hline 86 & 2 & 2 & 2 & 2 & 0 & 0 & 2 & 2 & 12 & 75.0 \\
\hline 89 & 2 & 2 & 2 & 2 & 0 & 0 & 2 & 2 & 12 & 75.0 \\
\hline 100 & 2 & 2 & 2 & 2 & 0 & 0 & 2 & 2 & 12 & 75.0 \\
\hline 106 & 2 & 2 & 2 & 2 & 0 & 0 & 2 & 2 & 12 & 75.0 \\
\hline 113 & 2 & 2 & 2 & 2 & 0 & 2 & 2 & 2 & 14 & 87.5 \\
\hline 117 & 2 & 2 & 2 & 2 & 2 & 2 & 2 & 2 & 16 & 100.0 \\
\hline 118 & 2 & 2 & 2 & 2 & 0 & 0 & 2 & 2 & 12 & 75.0 \\
\hline 130 & 2 & 2 & 2 & 2 & 0 & 0 & 2 & 2 & 12 & 75.0 \\
\hline 133 & 2 & 2 & 2 & 2 & 2 & 2 & 2 & 2 & 16 & 100.0 \\
\hline 136 & 2 & 2 & 2 & 2 & 0 & 0 & 2 & 2 & 12 & 75.0 \\
\hline 137 & 1 & 2 & 2 & 2 & 0 & 0 & 2 & 2 & 11 & 68.8 \\
\hline 146 & 1 & 2 & 2 & 2 & 0 & 0 & 2 & 2 & 11 & 68.8 \\
\hline 147 & 2 & 2 & 2 & 2 & 1 & 2 & 2 & 2 & 15 & 93.8 \\
\hline 149 & 0 & 2 & 2 & 2 & 0 & 0 & 2 & 2 & 10 & 62.5 \\
\hline 159 & 1 & 2 & 2 & 2 & 0 & 0 & 2 & 2 & 11 & 68.8 \\
\hline 162 & 1 & 2 & 2 & 2 & 1 & 2 & 2 & 2 & 14 & 87.5 \\
\hline 167 & 2 & 2 & 2 & 2 & 0 & 0 & 2 & 2 & 12 & 75.0 \\
\hline 169 & 2 & 2 & 2 & 2 & 0 & 0 & 2 & 2 & 12 & 75.0 \\
\hline 171 & 2 & 2 & 2 & 2 & 0 & 0 & 2 & 2 & 12 & 75.0 \\
\hline 176 & 2 & 2 & 2 & 2 & 0 & 0 & 2 & 2 & 12 & 75.0 \\
\hline
\end{tabular}

Studies are described using serial numbers in Table 3 
Table 9 Results of quality assessment of 181 studies — case series studies: 2 studies. The Joanna Briggs Institute Critical Appraisal Checklist is used

Scoring: Yes $=2 /$ Unclear $=1 /$ No $=0 / \mathrm{NA}=$ not applicable

Q1: Were there clear criteria for inclusion in the case series?

Q2: Was the condition measured in a standard, reliable way for all participants included in the case series?

Q3: Were valid methods used for identification of the condition for all participants included in the case series?

Q4: Did the case series have consecutive inclusion of participants?

Q5: Did the case series have complete inclusion of participants?

Q6: Was there clear reporting of the demographics of the participants in the study?

Q7: Was there clear reporting of clinical information of the participants?

Q8: Were the outcomes or follow-up results of cases clearly reported?

Q9: Was there clear reporting of the presenting site(s)/clinic(s) demographic information?

Q10: Was statistical analysis appropriate?

\begin{tabular}{|c|c|c|c|c|c|c|c|c|c|c|c|c|}
\hline Study (serial no.) & Q1 & 1. Q2 & 1. Q3 & 1. Q4 & 1. Q5 & 1. Q6 & 1. Q7 & 1. Q8 & 1. Q9 & 10. Q10 & Total ( /20) & $\%$ \\
\hline 76 & 1.2 & 2.2 & 2.1 & 2.1 & 1.2 & 1.2 & 1.2 & 1.1 & 2.2 & 11.2 & 17 & 85.0 \\
\hline 181 & 2. 2 & 3.2 & 3.2 & 3.2 & 2.2 & 2.2 & 2.2 & 2.2 & 3.2 & 12.2 & 20 & 100.0 \\
\hline
\end{tabular}

Studies are described using serial numbers in Table 3

Table 10 Results of quality assessment of 181 studies — randomised controlled trials: 40 studies. A modified version of critical appraisal checklist by van Tulder et al [15] is used

Scoring: Yes $=2 /$ Unclear $=1 /$ No $=0 /$ NA $=$ not applicable

$\mathrm{Q} 1:$ Acceptable method of randomisation

Q2: Concealed treatment allocation

Q3: Similar group values at baseline

Q4: Blinded assessor

Q5: No or similar co-interventions

Q6: Acceptable compliance $(\geq 75 \%)$

Q7: Acceptable drop-out rate $(<30 \%)$

Q8: Similar timing of the outcome assessment in all groups

Q9: Intention to treat analysis

Study (serial no.) Q

8

13

19

25

26

27

43

52

60

61
64

\begin{tabular}{|c|c|c|}
\hline Q9 & Total $(/ 18)$ & $\%$ \\
\hline 0 & 16 & 88.9 \\
\hline 2 & 18 & 100.0 \\
\hline 0 & 16 & 88.9 \\
\hline 0 & 16 & 88.9 \\
\hline 0 & 14 & 77.8 \\
\hline 0 & 14 & 77.8 \\
\hline 0 & 13 & 72.2 \\
\hline 2 & 17 & 94.4 \\
\hline 0 & 16 & 88.9 \\
\hline 0 & 12 & 66.7 \\
\hline 0 & 16 & 88.9 \\
\hline 0 & 11 & 61.1 \\
\hline 0 & 12 & 66.7 \\
\hline 0 & 16 & 88.9 \\
\hline 0 & 11 & 61.1 \\
\hline 0 & 16 & 88.9 \\
\hline 0 & 14 & 77.8 \\
\hline 0 & 14 & 77.8 \\
\hline 0 & 14 & 77.8 \\
\hline 0 & 16 & 88.9 \\
\hline 0 & 14 & 77.8 \\
\hline 2 & 15 & 83.3 \\
\hline 0 & 13 & 72.2 \\
\hline 0 & 15 & 83.3 \\
\hline 0 & 12 & 66.7 \\
\hline 0 & 15 & 83.3 \\
\hline 0 & 14 & 77.8 \\
\hline 0 & 15 & 83.3 \\
\hline 0 & 15 & 83.3 \\
\hline 0 & 15 & 83.3 \\
\hline 0 & 15 & 83.3 \\
\hline 0 & 16 & 88.9 \\
\hline 0 & 16 & 88.9 \\
\hline 0 & 15 & 83.3 \\
\hline 0 & 15 & 83.3 \\
\hline 0 & 12 & 66.7 \\
\hline 0 & 13 & 72.2 \\
\hline 0 & 16 & 88.9 \\
\hline 0 & 11 & 61.1 \\
\hline 2 & 18 & 100.0 \\
\hline
\end{tabular}

Studies are described using serial numbers in Table 3 
FACTOR

Case series study

Cross sectional study

Case-control study

Cohort study

Randomised controlled trial
Not-FACTOR

Case series study

Cross sectional study

Case-control study

Cohort study

Randomised controlled trial

a

Patients' demographical factors

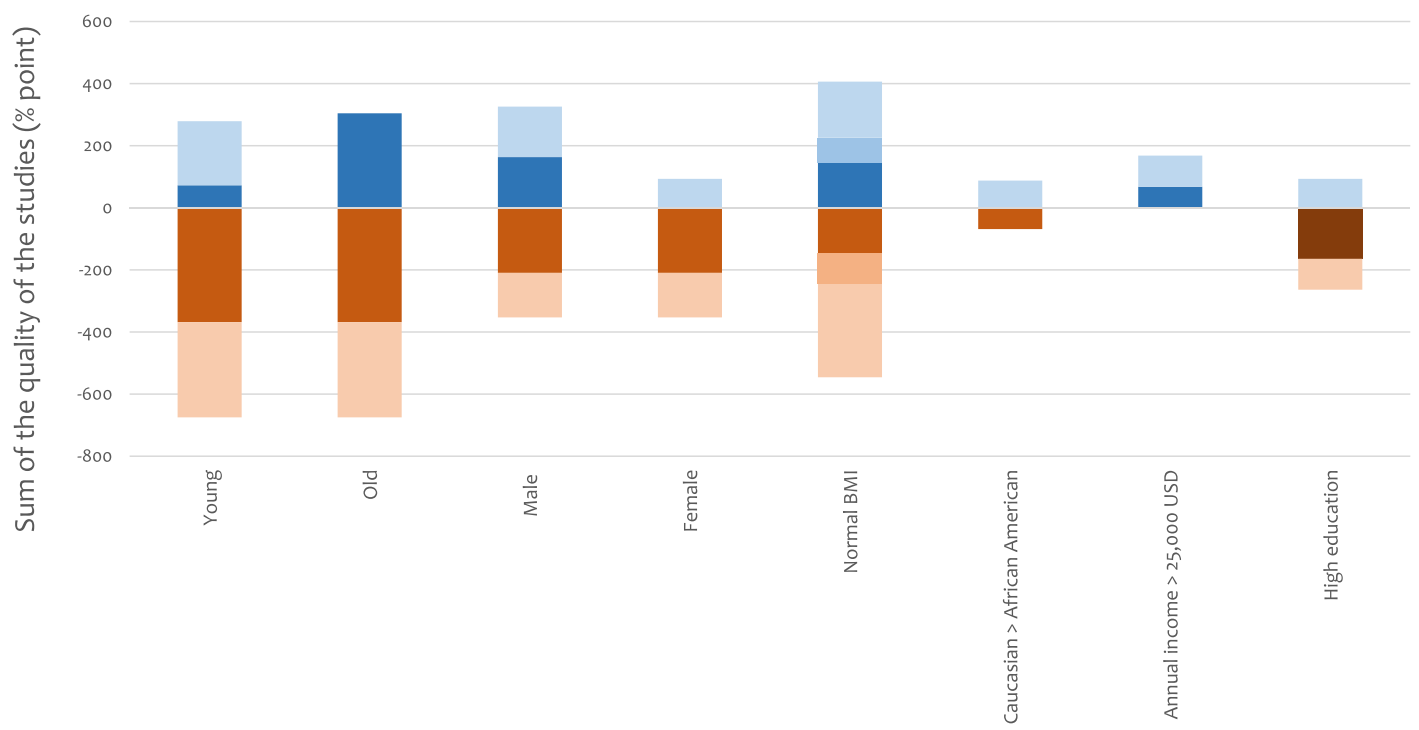

b Patients' non-knee factors

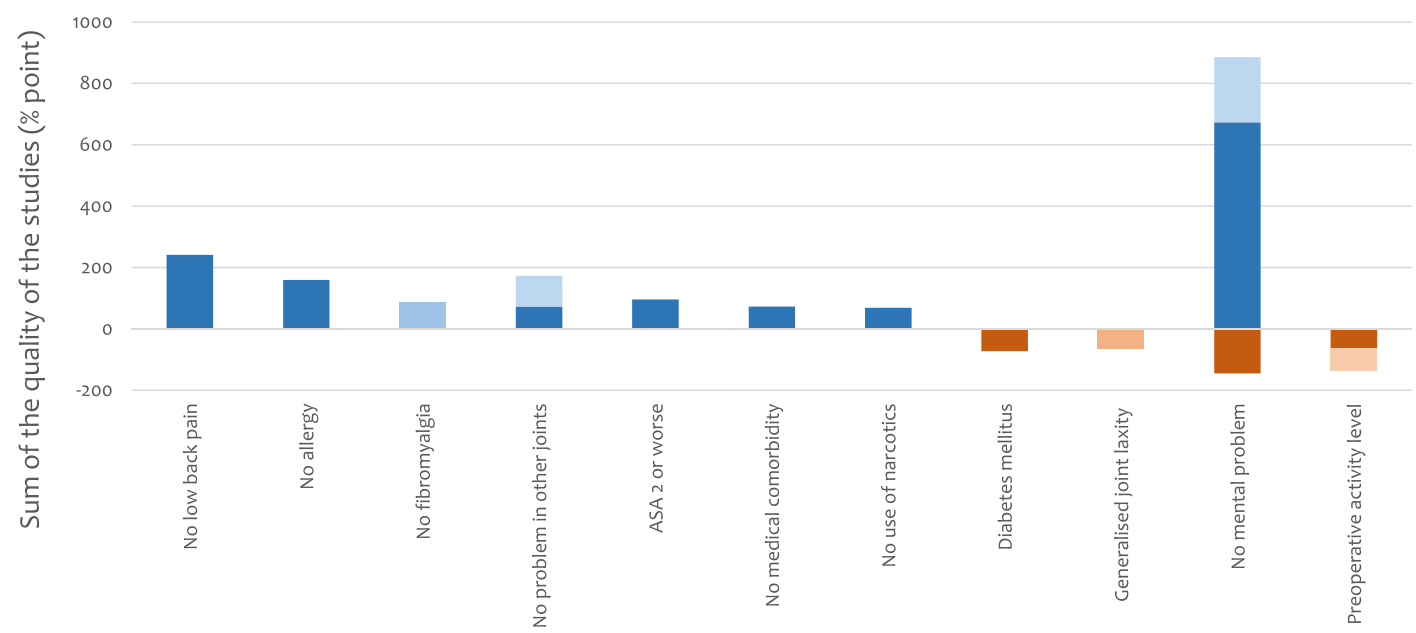

Fig. 3 Sum of percentage from full score (\%) based on the quality assessment in each type of study for each factor. Blue bar means FACTOR ('it is a factor for patients' satisfaction') and orange bar satisfaction'). a Patients' demographical factors. b Patients' non-knee factors. c Patients' knee factors. d Factors of implants/prostheses. e Intra-operative technical factors. f Post-operative outcome factors. $\mathbf{g}$ means Not-FACTOR ('it is a factor which does NOT relate to patients' 

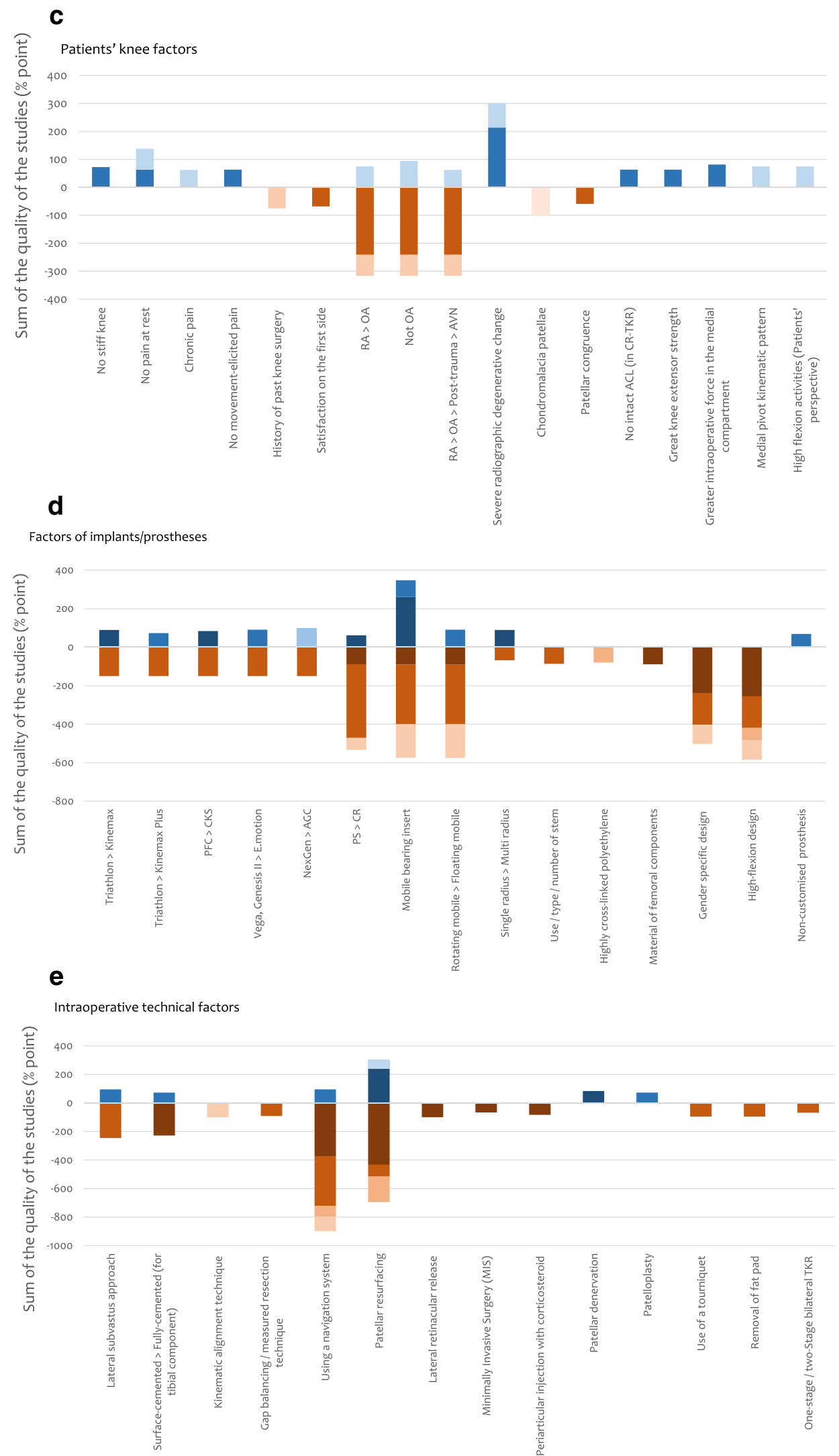

Fig. 3 (continued) 


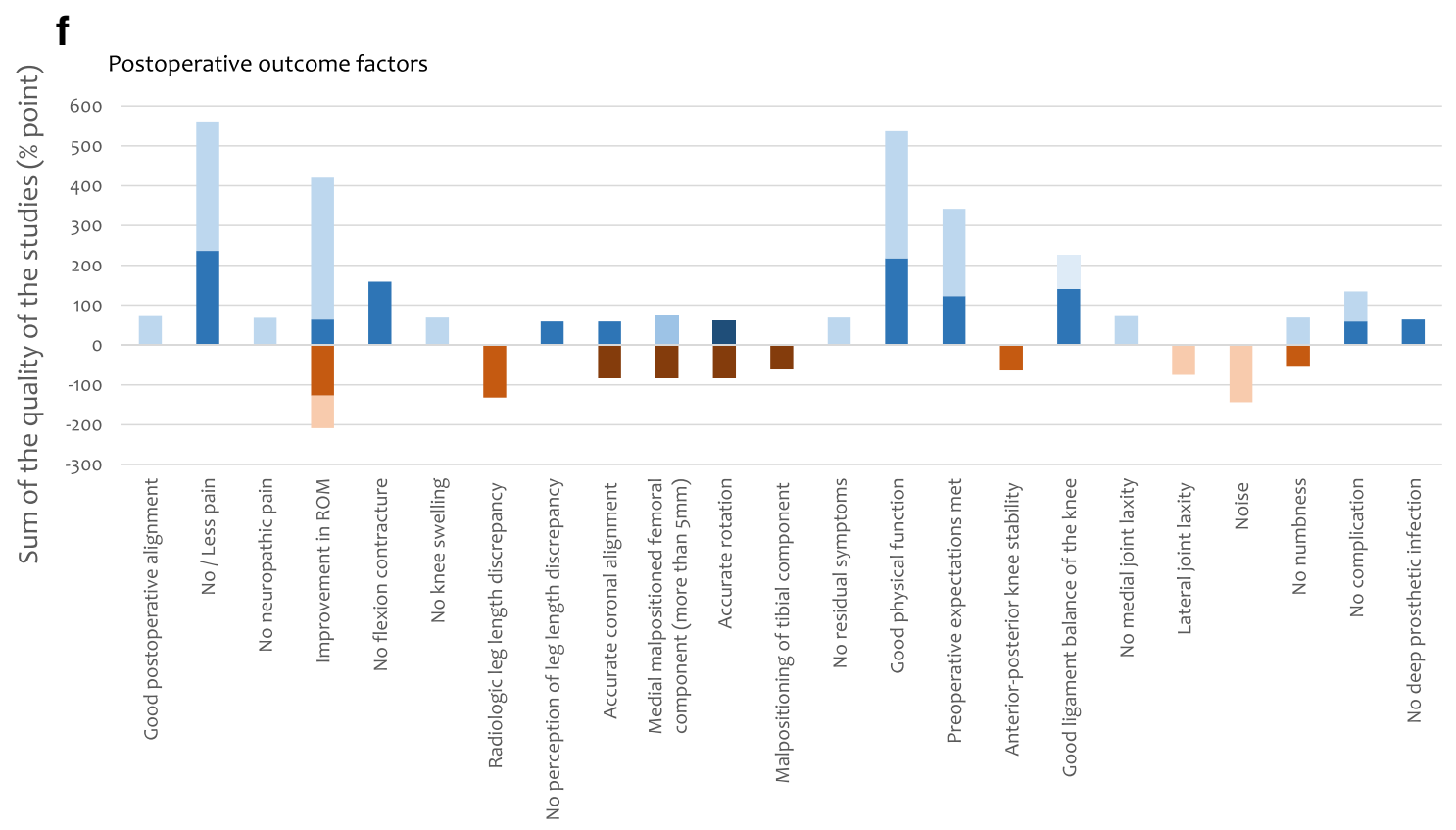

g Surgeon and healthcare factors

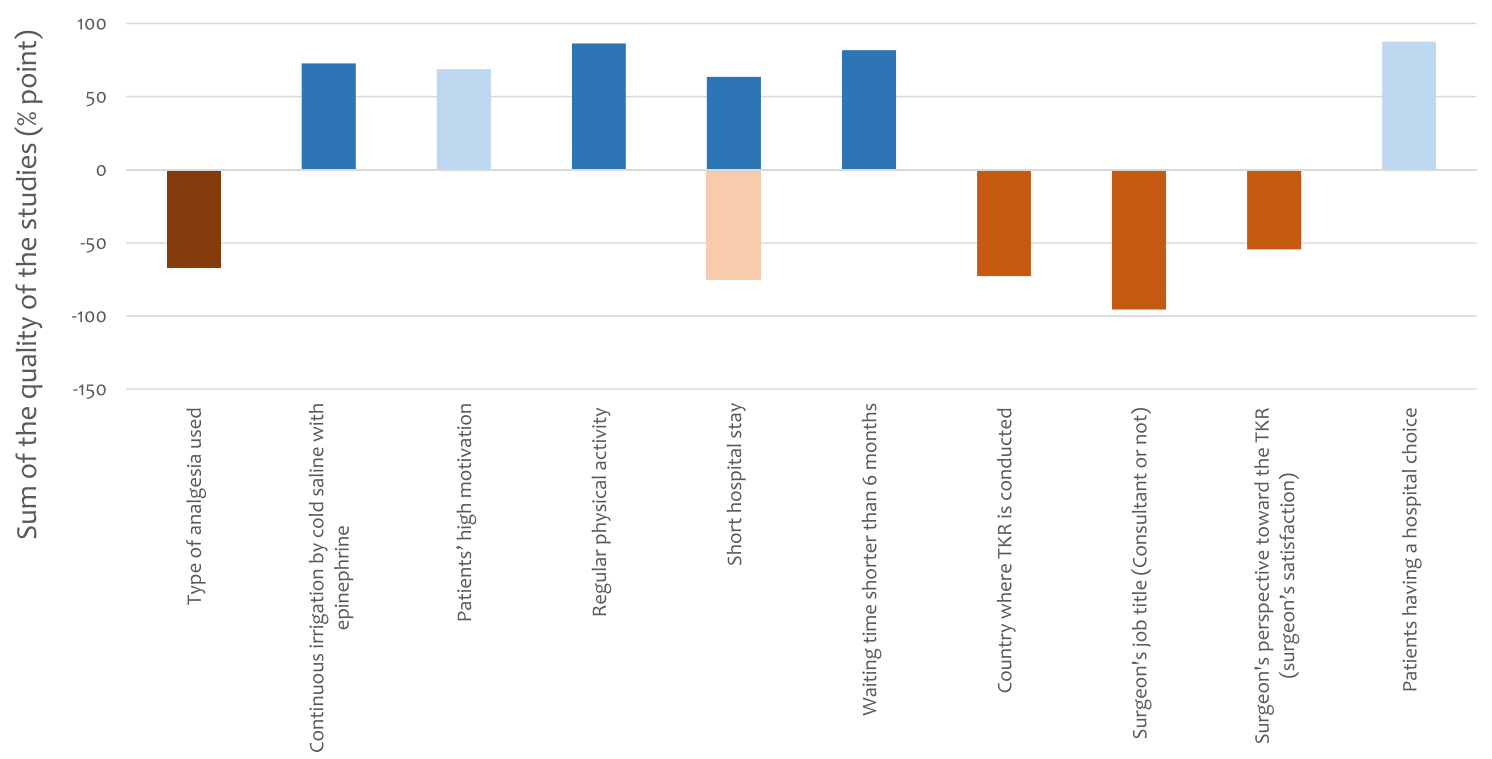

Fig. 3 (continued)

Table 11 List of frequently reported factors as FACTOR ('it is a factor for patient satisfaction')

Factors (number of reportings)

1st place

2nd place

2nd place

4th place

5th place

5th place uniform way of assessing satisfaction is essential for the orthopaedic community. Secondly, the timing of assessment of satisfaction after the index TKR varied amongst studies and this again requires standardisation. Thirdly, in many of the studies included in this review, the authors have only focused on one factor and the mutual or overall effect of multiple factors was not assessed. Fourthly, no statistical tests of intra-class correlation coefficients, inter-rater reliability and heterogeneity amongst the studies were performed in this systematic review. Finally, there are several studies in which patients are duplicated amongst studies and our review was 
Table 12 List of factors which have the highest sum of percentage score (a percentage from full score) of FACTOR ('it is a factor for patient satisfaction') only based on the quality assessment for various combination of the types of the studies

\begin{tabular}{|c|c|c|c|c|c|}
\hline & RCT & $\begin{array}{l}\text { RCT } \\
+ \text { Cohort }\end{array}$ & $\begin{array}{l}\text { RCT } \\
+ \text { Cohort } \\
+ \text { Case-control }\end{array}$ & $\begin{array}{l}\text { RCT } \\
+ \text { Cohort } \\
+ \text { Case-control } \\
+ \text { Cross-sectional }\end{array}$ & $\begin{array}{l}\text { RCT } \\
+ \text { Cohort } \\
+ \text { Case-control } \\
+ \text { Cross-sectional } \\
+ \text { Case series }\end{array}$ \\
\hline $\begin{array}{l}\text { 1st } \\
\text { place }\end{array}$ & $\begin{array}{l}\text { Use of mobile bearing } \\
\text { insert }(261.1 \%)\end{array}$ & $\begin{array}{l}\text { No mental health problems } \\
(672.6 \%)\end{array}$ & $\begin{array}{l}\text { No mental health problems } \\
\quad(672.6 \%)\end{array}$ & $\begin{array}{l}\text { No mental health } \\
\text { problems }(885.2 \%)\end{array}$ & $\begin{array}{l}\text { No mental health } \\
\text { problems }(885.2 \%)\end{array}$ \\
\hline $\begin{array}{l}\text { 2nd } \\
\text { place }\end{array}$ & $\begin{array}{l}\text { Patellar resurfacing } \\
\quad(238.9 \%)\end{array}$ & $\begin{array}{l}\text { Use of mobile-bearing } \\
\text { insert }(347.5 \%)\end{array}$ & $\begin{array}{l}\text { Use of mobile bearing } \\
\text { insert }(347.5 \%)\end{array}$ & $\begin{array}{l}\text { No/less post-operative } \\
\text { pain }(561.5 \%)\end{array}$ & $\begin{array}{l}\text { No/less post-operative } \\
\text { pain }(561.5 \%)\end{array}$ \\
\hline
\end{tabular}

$R C T$ randomised controlled trial

limited to publications in English, so there is a possibility of publication bias.

However, despite all these limitations, the main strength of this study lies in its broad and comprehensive initial literature search as well as complete and in-depth quality assessment for each study and the factors. We have determined all the factors which could potentially affect patient satisfaction following a TKR which have been reported in the literature thus far.

\section{Conclusion}

No history of mental health problems, use of a mobile bearing insert, patellar resurfacing, severe pre-operative radiological degenerative change, no low back pain, normal BMI, no/less post-operative pain, good physical function post-operatively, improvement in ROM and pre-operative expectations being met were considered to be significant factors leading to better patient satisfaction following a TKR.

Surgeons performing a TKR should take these factors into consideration prior to deciding whether a patient is suitable for a TKR. Secondarily, a detailed explanation of these factors should form part of the process of informed consent to achieve better patient satisfaction following TKR.
There is great need for a unified approach to assessing satisfaction following a TKR and also the time at which satisfaction is assessed.

Moreover, further studies and ideally larger RCTs focusing on each of these factors are required to determine the exact correlation of these factors with satisfaction.

\section{Compliance with ethical standards}

Conflict of interest The authors declare that they have no conflict of interest.

Ethical approval This article does not contain any studies with human participants or animals performed by any of the authors.

Open Access This article is licensed under a Creative Commons Attribution 4.0 International License, which permits use, sharing, adaptation, distribution and reproduction in any medium or format, as long as you give appropriate credit to the original author(s) and the source, provide a link to the Creative Commons licence, and indicate if changes were made. The images or other third party material in this article are included in the article's Creative Commons licence, unless indicated otherwise in a credit line to the material. If material is not included in the article's Creative Commons licence and your intended use is not permitted by statutory regulation or exceeds the permitted use, you will need to obtain permission directly from the copyright holder. To view a copy of this licence, visit http://creativecommons.org/licenses/by/4.0/.

Table 13 List of factors which have the highest sum of percentage score (a percentage from full score) of FACTOR ('it is a factor for patient satisfaction') and Not-FACTOR ('it is a factor which does NOT relate to patient satisfaction') based on the quality assessment for all type of the studies

\begin{tabular}{ll}
\hline & Factors (\% score) \\
\hline 1st place & No mental health problems $(739.8 \%)$ \\
2nd place & No/less post-operative pain $(561.5 \%)$ \\
3rd place & Good physical function $(536.9 \%)$ \\
4th place & Pre-operative expectations met (341.5\%) \\
5th place & Severe pre-operative radiographic degenerative change (301.2\%) \\
6th place & No low back pain (240.9\%) \\
\hline
\end{tabular}

Percentage score of Not-FACTOR was calculated as negative value 


\section{References}

1. NICE report: Osteoarthritis: the care and management of osteoarthritis. https://www.nice.org.uk/guidance/cg177/resources/ osteoarthritis-care-and-management-pdf-35109757272517. (date last accessed 16 Dec 2018)

2. Kurtz S, Ong K, Lau E, Mowat F, Halpern M (2007) Projections of primary and revision hip and knee arthroplasty in the United States from 2005 to 2030. J Bone Joint Surg Am 89(4):780-785

3. NJR Annual reports 2016. http://www.njrcentre.org.uk/njrcentre/ Reports,PublicationsandMinutes/Annualreports/tabid/86/Default. aspx (date last accessed 16 Dec 2018)

4. Insall JN, Dorr LD, Scott RD, Scott WN (1989) Rationale of the Knee Society clinical rating system. Clin Orthop Relat Res 248:1314

5. Hakim J, Volpin G, Amashah M, Alkeesh F, Khamaisy S, Cohen M, Ownallah J (2019) Long-term outcome of total knee arthroplasty in patients with morbid obesity. Int Orthop

6. Cho KJ, Seon JK, Jang WY, Park CG, Song EK (2019) Robotic versus conventional primary total knee arthroplasty: clinical and radiological long-term results with a minimum follow-up of ten years. Int Orthop 43(6):1345-1354

7. Bullens PHJ, Van Loon CJM, De Waal Malefijt MC, Laan RFJM, Veth RPH (2001) Patient satisfaction after total knee arthroplasty: a comparison between subjective and objective outcome assessments. J Arthroplast 16(6):740-747

8. Hawker GA (2006) Who, when, and why total joint replacement surgery? The patient s perspective. Curr Opin Intern Med 5(6):639643

9. Peersman G, Verhaegen J, Favier B (2019) The forgotten joint score in total and unicompartmental knee arthroplasty: a prospective cohort study. Int Orthop

10. Sugita T, Miyatake N, Aizawa T, Sasaki A, Kamimura M, Takahashi A (2018) Quality of life after staged bilateral total knee arthroplasty: a minimum five-year follow-up study of seventy-eight patients. Int Orthop

11. Robertsson O, Dunbar M, Pehrsson T, Knutson K, Lidgren L (2000) Patient satisfaction after knee arthroplasty: a report on 27, 372 knees operated on between 1981 and 1995 in Sweden. Acta Orthop Scand 71(3):262-267

12. Baker PN, Rushton S, Jameson SS, Reed M, Gregg P, Deehan DJ (2013) Patient satisfaction with total knee replacement cannot be predicted from pre-operative variables alone: a cohort study from the National Joint Registry for England and Wales. Bone Joint $\mathrm{J}$ 95B(10):1359-1365

13. Moher D, Liberati A, Tetzlaff J, Altman DG (2009) Preferred Reporting Items for Systematic Reviews and Meta-Analyses: the PRISMA statement. PLoS Med 6(7):e1000097

14. JBI JBI-CAT. Critical appraisal tools. http://joannabriggs.org/ research/critical-appraisal-tools.html (date last accessed 16 Dec 2018)

15. van Tulder M, Furlan A, Bombardier C, Bouter L (2003) Updated method guidelines for systematic reviews in the Cochrane Collaboration Back Review Group. Spine (Phila Pa 1976) 28(12): 1290-1299

16. Brander V, Gondek S, Martin E, Stulberg SD (2007) Pain and depression influence outcome 5 years after knee replacement surgery. Clin Orthop Relat Res 464:21-26

17. Bonnin MP, Basiglini L, Archbold HA (2011) What are the factors of residual pain after uncomplicated TKA? Knee Surg Sports Traumatol Arthrosc 19(9):1411-1417

18. Macleod U, Mitchell E, Black M, Spence G (2004) Comorbidity and socioeconomic deprivation: an observational study of the prevalence of comorbidity in general practice. Eur J Gen Pract 10(1): 24-26
19. Verra ML, Angst F, Staal JB et al (2011) Differences in pain, function and coping in Multidimensional Pain Inventory subgroups of chronic back pain: a one-group pretest-posttest study. BMC Musculoskelet Disord 12:145

20. Carothers JT, Kim RH, Dennis DA et al (2011) Mobile-bearing total knee arthroplasty: a meta-analysis. J Arthroplast 26(4):537542

21. Price AJ, Rees JL, Beard D et al (2003) A mobile-bearing total knee prosthesis compared with a fixed-bearing prosthesis: a multicentre single-blind randomised controlled trial. J Bone Joint Surg (Br) 85B:62-67

22. Shakespeare D, Kinzel V, Ledger M (2005) Achieving ligament stability and correct rotational alignment of the femur in knee arthroplasty: a study using the Medial Pivot knee. Knee 12:419 423

23. Kim YH, Yoon SH, Kim JS (2009) Early outcome of TKA with a medial pivot fixed-bearing prosthesis is worse than with a PFC mobile-bearing prosthesis. Clin Orthop Relat Res 467(2):493-503

24. Mayman D, Bourne RB, Rorabeck CH, Vaz M, Kramer J (2003) Resurfacing versus not resurfacing the patella in total knee arthroplasty: 8- to 10-year results. J Arthroplast 18:541-545

25. Roberts DW, Hayes TD, Tate CT, Lesko JP (2015) Selective patellar resurfacing in total knee arthroplasty: a prospective, randomized, double-blind study. J Arthroplast 30(2):216-222

26. Waters TS, Bentley G (2003) Patellar resurfacing in total knee arthroplasty. A prospective, randomized study. J Bone Joint Surg Am 85-A(2):212-217

27. Burnett RS, Haydon CM, Rorabeck CH, Bourne RB (2004) The John Insall Award : patella resurfacing versus nonresurfacing in total knee arthroplasty. Clin Orthop Relat Res 428:12-25

28. Burnett RSJ, Boone JL, Rosenzweig SD, Steger-May K, Barrack RL (2009) Patellar resurfacing compared with nonresurfacing in total knee arthroplasty: a concise follow-up of a randomized trial. J Bone Joint Surg Am 91(11):2562-2567

29. Tanzer M, McLean CA, Laxer E et al (2001) Effect of femoral component designs on the contact and tracking characteristics of the unresurfaced patella in total knee arthroplasty. Can J Surg 44: $127-133$

30. Benjamin JB, Szivek JA, Hammond AS et al (1998) Contact areas and pressures between native patellas and prosthetic femoral components. J Arthroplast 13:693-698

31. Andriacchi TP, Yoder D, Conley A et al (1997) Patellofemoral design influences function following total knee arthroplasty. J Arthroplast 12:243-249

32. Kellgren JH, Lawrence JS (1957) Radiological assessment of osteoarthrosis. Ann Rheum Dis 16(4):494-502

33. Riddle DL, Jiranek WA, Neff RS, Whitaker D, Hull JR (2012) Extent of tibiofemoral osteoarthritis before knee arthroplasty: multicenter data from the osteoarthritis initiative. Clin Orthop Relat Res 470(10):2836-2842

34. Keurentjes JC, Fiocco M, So-Osman C et al (2013) Patients with severe radiographic osteoarthritis have a better prognosis in physical functioning after hip and knee replacement: a cohort-study. PLoS One 8(4):e59500

35. Meding JB, Ritter MA, Faris PM, Keating EM, Harris W (2001) Does the preoperative radiographic degree of osteoarthritis correlate to results in primary total knee arthroplasty? J Arthroplast 16(1):13-16

36. Wolterbeek N, Garling EH, Mertens BJ, Nelissen RG, Valstar ER (2012) Kinematics and early migration in single-radius mobile- and fixed-bearing total knee prostheses. Clin Biomech (Bristol, Avon) 27(4):398-402

37. Clement ND, MacDonald D, Simpson AHRW, Burnett R (2013) Total knee replacement in patients with concomitant back pain results in a worse functional outcome and a lower rate of satisfaction. Bone Joint J 95-B(12):1632-1639 
38. Singh JA, O'Byrne M, Harmsen S, Lewallen D (2010) Predictors of moderate-severe functional limitation after primary total knee arthroplasty (TKA): 4701 TKAs at 2-years and 2935 TKAs at 5years. Osteoarthr Cartil 18(4):515-521

39. Baker P, Muthumayandi K, Gerrand C, Kleim B, Bettinson K, Deehan D (2013) Influence of body mass index (BMI) on functional improvements at 3 years following total knee replacement: a retrospective cohort study. PLoS One 8(3):e59079
40. Dailiana ZH, Papakostidou I, Varitimidis S et al (2015) Patientreported quality of life after primary major joint arthroplasty: a prospective comparison of hip and knee arthroplasty. BMC Musculoskelet Disord 16:366

Publisher's note Springer Nature remains neutral with regard to jurisdictional claims in published maps and institutional affiliations. 